OECD Working Papers on Public Governance No. 45

The OECD Framework for digital talent and skills OECD in the public sector 


\section{PUBLIC GOVERNANCE DIRECTORATE PUBLIC GOVERNANCE COMMITTEE}

Cancels \& replaces the same document of 10 November 2020

\section{OECD Digital Government Project}

\section{The OECD Framework for Digital Talent and Skills in the public sector}

This draft of The OECD Framework for Digital Talent and Skills in the public sector has been developed by the OECD Digital Government and Data Unit, within the Public Governance Directorate (GOV) Open and Innovative Government Division (OIG).

It has been developed in coordination with the OECD Working Party of Senior Digital Government Officials (E-leaders) and the Thematic Group on Digital Talents and Skills.

Delegates are invited to approve and comment on the document by December $\mathbf{1}^{\text {st }}, \mathbf{2 0 2 0}$.

Barbara Ubaldi (barbara.ubaldi@oecd.org)

Benjamin Welby (benjamin.welby@oecd.org)

Lucia Chauvet (lucia.chauvet@oecd.org)

\section{JT03478574}


2 | GOV/PGC/EGOV(2020)9

\section{OECD REGULATORY POLICY WORKING PAPERS}

The statistical data for Israel are supplied by and under the responsibility of the relevant Israeli authorities. The use of such data by the OECD is without prejudice to the status of the Golan Heights, East Jerusalem and Israeli settlements in the West Bank under the terms of international law. 


\section{Abstract}

This working paper presents the OECD Framework for Digital Talent and Skills in the Public Sector, a three-pillar framework for equipping the public sector (whether national or local) with the skills to achieve digital government maturity. Pillar 1 covers the importance of the context for those working on digital government and discusses the environment required to encourage digital transformation. Pillar 2 addresses the skills to support digital government maturity, covering all public servants, particular professionals and those in leadership roles. Pillar 3 considers the practical steps and enabling activities required to establish and maintain a workforce that encompasses the skills to support digital government maturity. 


\section{Acknowledgements}

This working paper was prepared by the Directorate for Public Governance (GOV), under the leadership of Director Elsa Pilichowski.

This paper was produced by GOV's Open and Innovative Government Division (OIG) under the guidance of Barbara Ubaldi, Head of the Digital Government and Data Unit. Chapters 2 and 4 were written by Lucia Chauvet, Junior Policy Analyst, and Chapter 3 was written by Benjamin Welby, Policy Analyst from the Digital Government and Data Unit. All chapters benefitted from insightful comments and support from Daniel Gerson, Head of Public Employment and Management, Public Management and Budgeting Division, and Kent Aitken, Policy Analyst in the Observatory of Public Sector Innovation (OPSI) team with Tatyana Teplova, Head of Gender, Justice and Inclusiveness Division providing a final editorial review.

The authors are thankful to colleagues from within the OECD for their feedback and comments, including Andrew Bell, Samuel Kim, Francesca Borgonovi, Bart Staats and Rasa Silyte-Niavas from the Center for Skills, Natalie Laechelt from the Education and Skills Directorate, and Jacob Arturo Rivera Perez, Felipe Gonzalez-Zapata, Cecilia Emilsson, Mariane Piccinin Barbieri, Ethel Tan and Alex Seemann from GOV.

The OECD wishes to acknowledge the ongoing support of the OECD Working Party of Senior Digital Government Officials (E-Leaders) and the OECD Expert Group on Digital Talent and Skills, led by Italy and benefitting from the input of Australia, Canada, Colombia, Estonia, Italy, Portugal, Singapore, Slovenia, Spain, the United Kingdom and Uruguay. We also would like to give special thanks to Australia, Estonia, Slovenia and the United Kingdom for their inputs to the working paper. 


\section{Table of contents}

Abstract 2

Acknowledgements 4

Executive Summary $\quad 7$

1 Introduction 9

The world is digital and it needs a digitally enabled state 9

Building on the OECD's work on skills 11

The OECD Framework for Digital Talent and Skills in the public sector 16

2 The right environment to encourage digital transformation 18

Leadership 20

Organisational structure $\quad 22$

Learning culture $\quad 26$

Ways of working $\quad 28$

3 Defining skills for a digitally enabled state $\quad 31$

$21^{\text {st }}$ century skills in society $\quad 32$

Digital government user skills $\quad 34$

Digital government socio-emotional skills $\quad 42$

Digital government professional skills 44

Digital government leadership skills 46

4 Path to a digital workforce $\quad 49$

Attracting talents $\quad 50$

Retaining talents $\quad 56$

Developing and maintaining skills $\quad 57$

$\begin{array}{ll}\text { Allocating talents and skills } & 61\end{array}$

$\begin{array}{ll}\text { Reforming the environment } & 63\end{array}$

5 Conclusion 65

$\begin{array}{ll}\text { Glossary } & 69\end{array}$

$\begin{array}{ll}\text { References } & 72\end{array}$ 


\section{6 | GOV/PGC/EGOV(2020)9}

\section{Tables}

Table 2.1. Leadership for creating an environment to encourage digital transformation 22

Table 2.2. Organisational structures for creating an environment to encourage digital transformation 24

Table 2.3. A learning culture that creates an environment to encourage digital transformation 27

Table 2.4. Ways of working that create an environment to encourage digital transformation 30

Table 3.1. Competencies for digital government leadership 4

Table 4.1. Attracting the talent for a digital workforce $\quad 54$

Table 4.2. Retaining the talent for a digital workforce $\quad 57$

Table 4.3. Developing and maintaining the skills of a digital workforce 61

Table 4.4. Allocating a digital workforce 63

\section{Figures}

Figure 1.1. The OECD Digital Government Policy Framework (DGPF) 9

Figure 1.2. The OECD Digital Government Index Composite Results, 2019

Figure 1.3. From societal, to organisational, to individual and team skills 11

Figure 1.4. Skills for a digital world $r$

Figure 1.5. Civil service skills for public value: a framework $\quad 14$

Figure 1.6. Six skills areas for public sector innovation $\quad 15$

Figure 1.7. Four leadership capabilities $\quad 16$

Figure 1.8. The OECD Framework for Digital Talent and Skills in the Public Sector 17

Figure 2.1. The OECD Framework for Digital Talent and Skills in the Public Sector (focus on Pillar 1) 18

Figure 3.1. The OECD Framework for Digital Talent and Skills in the Public Sector (focus on Pillar 2) 31

Figure 3.2. Skills to support digital government maturity

Figure 3.3. Digital government user skills $\quad 35$

Figure 3.4. Digital government socio-emotional skills and associated behaviours needed by teams 43

Figure 3.5. Professions involved in a multi-disciplinary team 45

Figure 4.1. The OECD Framework for Digital Talent and Skills in the Public Sector (focus on Pillar 3) 49

\section{Boxes}

Box 1.1. The 6 dimensions of the Digital Government Policy Framework 10

Box 2.1. Emergent approaches to delivery 19

Box 2.2. The Australian Public Service Review 23

Box 2.3. United Kingdom DDaT Profession Capability Framework: Service designer 25

Box 2.4. Building learning cultures within governments 27

Box 3.1. 5 areas of the European Digital Competence Framework explained 33

Box 3.2. Overarching principles for designing, and delivering, services 36

Box 3.3. Enablers to support the digital transformation of services at scale 37

Box 4.1. The Digital, Data and Technology Profession Capability Framework 52

Box 4.2. Pennsylvania state government recruitment process $\quad 53$

Box 4.3. Estonia's nudging methods to increase the share of women in ICT professions 55

Box 4.4. Legislation promoting gender equality in Portugal $\quad 55$

Box 4.5. Canada's Free Agents program $\quad 59$

Box 4.6. The United Kingdom's Technology Code of Practice $\quad 59$ 


\section{Executive Summary}

In light of the rapid advancement of technology and the potential that data offers, the way people live, learn, work, and interact is no longer the same. Society -- including industries, education and businesses -- is evolving and incorporating digital technology, tools and data in daily operations to deliver better outcomes. This digital disruption is no different for public sectors, which need to be equipped, not only with the right technology, but also with the right working environment, the right skills and the right talents to support the progression from e-government to digital government. Achieving a digital workforce is critical for unlocking the potential of digital technologies and data to better meet citizen needs and build public trust in line with the 2014 OECD Recommendation of the Council on Digital Government Strategies.

The implications of the COVID-19 pandemic have emphasised the importance of these efforts for ensuring governments have the competency to operate in an increasingly digital world. The multifaceted crisis has provoked a massive demand shock across the public sector and increased the pressure on human resource leaders to ensure their organisations have skills that are fit for the $21^{\text {st }}$ century and resilient to economic and social shocks.

There are no simple solutions for transforming analogue organisations into digital ones; it isn't possible, or desirable, to assume that you might replace an existing workforce with one that is more 'digitally savvy'. Instead, public sectors need to embed a change in culture, supported by holistic strategies that revamp the working environment to encourage flexibility and adaptability; empower and enable their staff to be proactive with a positive approach to change; and identify, understand and develop the necessary talent and skills for a digitally enabled public sector.

The OECD Framework for Digital Talent and Skills in the Public Sector presented in this working paper offers a three-pillar approach for guiding the public sector (whether national or local) to acquire digital talent and equip public servants with digital skills. The framework demonstrates that the starting point is creating an environment that encourages the workforce to lead the digital transformation (Chapter 2). Organisations need to consider all the elements that contribute to a digitally-enabling working environment such as leadership, organisational structure, learning culture and ways of working.

As a digitally suitable working environment is established, it must be complemented by defining the digital talent and skills that enable public servants to drive digital maturity (Chapter 3 ). The starting point for a digitally-enabled state is a society, including public servants, that is equipped with foundational $21^{\text {st }}$ century skills. The public sector can then invest in a comprehensive approach that covers four additional areas of skills to support digital government maturity:

- digital government user skills,

- digital government socio-emotional skills,

- digital government professional skills, and

- digital government leadership skills.

An administration that not only provides the right support but also identifies the skills needed for digital government must consider how it attracts, recruits, retains and develops a workforce that leads to greater 


\section{8 | GOV/PGC/EGOV(2020)9}

digital government maturity (Chapter 4). Human resources in the public sector need to adapt and use new means of attracting and retaining employees as well as develop career paths that promote a learning culture both formally and informally. In this way, there is a feedback loop between the environment (Chapter 2) that sets the organisational culture and the application of practices to build a path to a digital workforce (Chapter 4).

This working paper shows pathways for developing a digital public workforce with the necessary skills to achieve a successful digital transformation. The following actions are suggested to guide governments in considering the contextual, skills and talent management aspects of digital transformation.

- Building the right environment for encouraging digital transformation requires leaders and their organisations to:

- be aware of the digital skills that a workforce requires to keep pace with digital evolution

- communicate a clear and understandable vision of the role of digital and actively champion the benefits of digital government

- demonstrate their engagement by visibly endorsing and actively participating in the rhythm of digital delivery, reducing hierarchical layers and delegating decision making by empowering teams as the unit of delivery

- focus on digital professions that are user-centred and have specific objectives and roles

- establish a learning culture that encourages and provides safety for employees to experiment

- support different ways of working with necessary policies, tools and technologies.

- Establishing the skills for a digitally enabled state requires:

- a broader digital skills strategy for society as a whole ensuring all are equipped with the necessary skills to thrive in the digital age

- public servants equipped with the digital user skills that support digital government maturity

- diverse and multidisciplinary teams consisting of well-trained digital and non-digital professionals reflecting a blend of digital government socio-emotional skills and behaviours to design and deliver trustworthy and proactive services with users' needs in mind

- leaders that model digital government user skills and actively shape the environment to create a digitally enabled state.

- Creating a path to a digital workforce requires:

- proactive recruitment strategies that promote the public sector as an attractive, trustworthy and transparent employer

- well-designed, fair, trusted and attractive reward systems that support clear career planning

- managers who emphasise job growth and professional development through multidisciplinary teams

- investment in digital talents by offering regular feedback loops and mentoring programmes, and providing training in both formal and informal ways

- encouraging job mobility and a diversity of career choices for public servants. 


\section{Introduction}

\section{The world is digital and it needs a digitally enabled state}

Digital technologies have completely changed the way in which economies operate, including their structure, how production is carried out, the breadth of consumer choice, the nature of labour markets and the need for new skills (Reljic, Evangelista and Pianta, 2019 $\left.{ }_{[1]}\right)$. As governments embrace the opportunities of digital technologies and data to improve their efficiency and effectiveness in anticipating, understanding and responding to the needs of the public, the nature of the work carried out by public servants is changing.

Governments, whether national or local, need to keep pace with citizens' expectations, manage increasing pressures on budgets and respond to new policy issues in order to maintain and restore trust. The COVID19 crisis presented a new challenge to levels of trust, but it also demonstrated the importance of a more flexible and adaptable public service in dealing with uncertainty. In analysing the impact of the COVID-19 crisis as a catalyst for government transformation, the OECD has found that governments with the digital government maturity to act as 'digitally enabled states' were better prepared to use digital technologies and data for managing the crisis and demonstrated greater resilience and responsiveness as a result (OECD, Forthcoming[2]; OECD, 2020[3]).

Creating a digitally enabled state goes beyond "e-government", which focuses on technology to improve efficiency in response to government needs. This evolution requires digital government practices that concentrate on designing policies and services to meet the needs of users. Building on the $O E C D$ Recommendation of the Council on Digital Government Strategies (OECD, 2014[4] $)$ this paradigm shift is measured by the Digital Government Index (DGI) against the Digital Government Policy Framework (DGPF) (Figure 2.1) (OECD, 2020[5]; OECD, 2020[6]), discussed in Box 2.1.

\section{Figure 2.1. The OECD Digital Government Policy Framework (DGPF)}

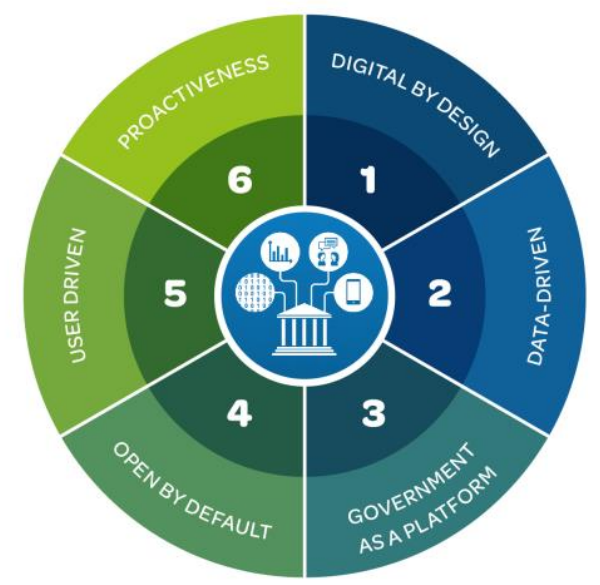

Source: Based on the OECD Recommendation of the Council on Digital Government Strategies (OECD, 2014[4]) 


\section{Box 2.1. The 6 dimensions of the Digital Government Policy Framework}

1. Digital by design: establishing clear organisational leadership, paired with effective coordination and enforcement mechanisms so that "digital" is considered not only as a technical topic, but as an embedded transformative element for rethinking and re-engineering public processes, simplifying procedures, and creating new channels of communication and engagement with public stakeholders.

2. Data-driven public sector: recognising data as a strategic asset and establishing the governance to generate public value through planning, delivering and monitoring public policies and services while adopting rules and ethical principles for trustworthy and safe access, sharing and re-use.

3. Government as a platform: building an ecosystem of guidelines, tools, data, standards and common components that equip teams to focus on user needs in public service design and delivery.

4. Open by default: making government data and policy-making processes (including algorithms) available for the public to engage with, within the limits of existing legislation and in balance with the national and public interest.

5. User-driven: awarding a central role to people' needs and convenience in the shaping of processes, services and policies; and by adopting inclusive mechanisms for this to happen.

6. Proactiveness: the ability of governments and civil servants to anticipate people's needs and respond to them rapidly, so that users do not have to engage with cumbersome processes associated with service delivery and data.

Source: OECD, (2020[5]), The OECD Digital Government Policy Framework: Six dimensions of a Digital Government

\section{Figure 2.2. The OECD Digital Government Index Composite Results, 2019}

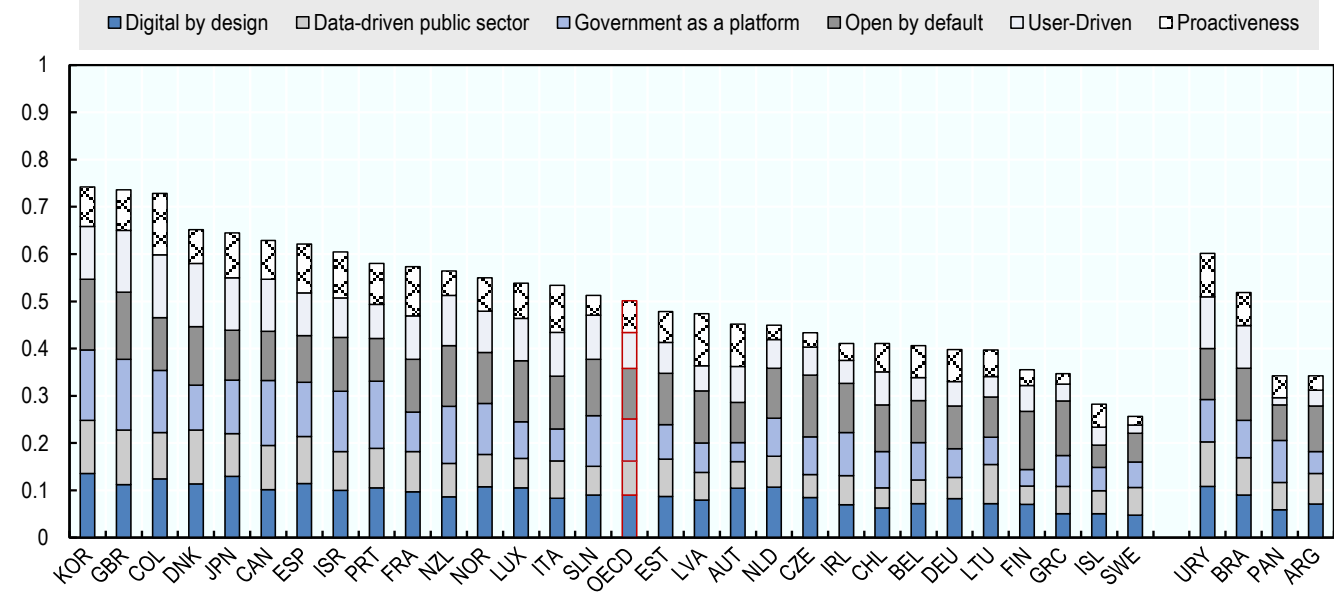

Note: Data are not available for Australia, Hungary, Mexico, Poland, Slovakia, Switzerland, Turkey and the United States of America. Information on data for Israel: http://dx.doi.org/10.1787/888932315602.

Source: OECD, (OECD, 2020[6]) Digital Government Index: 2019 results. 
The e-government era made significant progress in moving governments away from their analogue and paper-based past, and set new expectations for information and communication technology (ICT) skills in both the workforce and society in general. However, successfully developing maturity against the DGPF to make the transition to digital government requires ongoing evolution in skills as well as to the environment in which they are deployed.

There are no simple solutions for transforming analogue organisations into digital ones; it isn't possible, or desirable, to assume that you might replace an existing workforce with one that is more 'digitally savvy'. Instead, public sectors need to embed a change in culture, supported by holistic strategies that revamp the working environment to encourage flexibility and adaptability; empower and enable their staff to be proactive with a positive approach to change; and identify, understand and develop the necessary talent and skills for a digitally enabled public sector.

This Working Paper focuses on the role of public sector talent and skills in supporting and promoting greater digital government maturity in the national or local context. It draws on the experience of the the OECD Working Party of Senior Digital Government Officials (E-leaders) and examples drawn from the Thematic Group on Digital Talents and Skills ${ }^{1}$. The framework presented in this paper represents a model to underpin the shift to a digital government mindset and practice, supported by technical skills, that makes governments more open, collaborative, inclusive, innovative and sustainable.

\section{Building on the OECD's work on skills}

Before discussing the framework in more detail, it is important to situate the work on digital government and data within the body of OECD work exploring the challenge of developing skills and competencies in the digital age across the three tiers shown in Figure 2.3.

\section{Figure 2.3. From societal, to organisational, to individual and team skills}

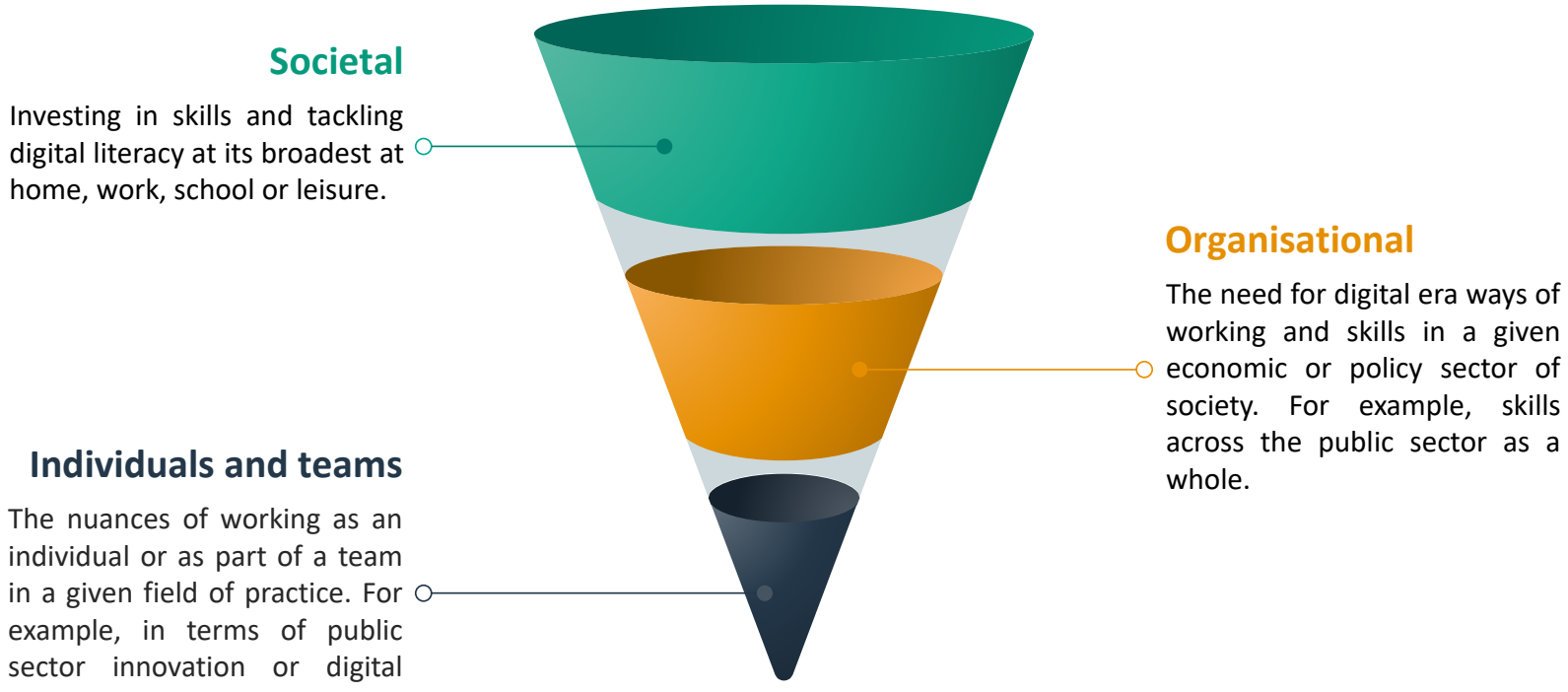

government.

Source: Authors' own design

\footnotetext{
${ }^{1}$ During the period of data collection, the Thematic Group was led by Italy and consisted of Australia, Canada, Colombia, Estonia, Italy, Portugal, Singapore, Slovenia, Spain, the United Kingdom and Uruguay
} 
The starting point for this discussion are the needs within society itself and efforts to understand skills and digital literacy at their broadest. The approach taken in society then shapes discussions within organisations about the needs for digital-era ways of working and skills in the context of their economic or policy sector. Finally, within those sectors and organisations are the discussions and frameworks that relate to individual- and team-level nuances of a given field of practice. It is to this third layer of skills, as they manifest for individuals and teams, that the framework presented in this paper contributes in considering the environment, skills and practices needed to ensure countries are well equipped to achieve digital government maturity

\section{Skills at the societal level}

The OECD's work on digital government and data builds on societal-level consideration of skills in the wider economy, the work of the OECD's Centre for Skills, and the OECD Future of Education and Skills 2030 project. The Centre supports countries in achieving better economic and social outcomes by taking an approach to developing and implementing skills policies that incorporates the whole of government. The OECD Skills Outlook captures this work, and helps understand the state of skills in society across different domains (OECD, 2017[7]; OECD, 2017[8]; OECD, 2019[9]). The OECD's Future of Education and Skills 2030 project provides an opportunity to explore the longer-term challenges facing education, and make the process of curriculum design and development more evidence-based and systematic (OECD, 2019[10]).

Technology has changed the way in which people live: with only a few clicks, people can communicate with those far away and more easily access information, services, education and work. With the potential of digital technologies to improve productivity and well-being, digital skills have become an increasingly prominent priority across countries -- a trend that accelerated, during the COVID-19 crisis.

Nevertheless, digital transformation does not automatically contribute to greater equality. Access to, or competencies in, digital technologies can differ among individuals, professions, economic sectors, companies and regions. Therefore, it is critical to secure the availability of adequate skills across society to meet the needs of citizens, release the potential brought by the digital age, and ensure that digital transformation is just and fair. Rather than waiting for the impact of technology on workers, society and economies, a more proactive approach should be taken to investing in skills, training and education to make the most of digital transformation (OECD, 2019 $\left.{ }_{[9]}\right)$. Indeed, good-quality training in digital and ICT for low-skilled workers has the potential to produce a 'double dividend': boosting productivity and economic output while bridging digital divides and reducing inequality (OECD, 2019[11] $)^{2}$.

The rapid evolution of technology makes it critical for students to "learn how to learn" as a lifelong capacity to respond to the transformation around them. This is more likely to happen when students are agents in their own learning. Therefore, it is important for education systems to develop the agency of students by ensuring they play an active role in deciding what and how they want to learn. This can have wider benefits through encouraging co-agency - the involvement of communities and peers - in creating environments that help to shape individuals and develop their talents (OECD, 2019 $[10])$.

In order to secure a comprehensive approach that provides the skills needed for societies to thrive in a digital world, Figure 2.4 shows how the OECD recommends augmenting core ideas of skills proficiency (in terms of technological, digital, cognitive and socio-emotional skills) with a recognition of context-specific skills needs in the workplace, society and education, supported by a comprehensive set of skills-related policies (OECD, 2019[9]).

\footnotetext{
2 Burns and Gottschalk (2019[91]) identify three categories of digital divide in their work on $21^{\text {st }}$ century childhood wellbeing: a first-level divide concerning access to the Internet; a second-level divide in terms of inequalities of skills and usage; and a third-level divide concerning the potentially magnifying effect of the Internet on existing offline inequalities.
} 
Figure 2.4. Skills for a digital world
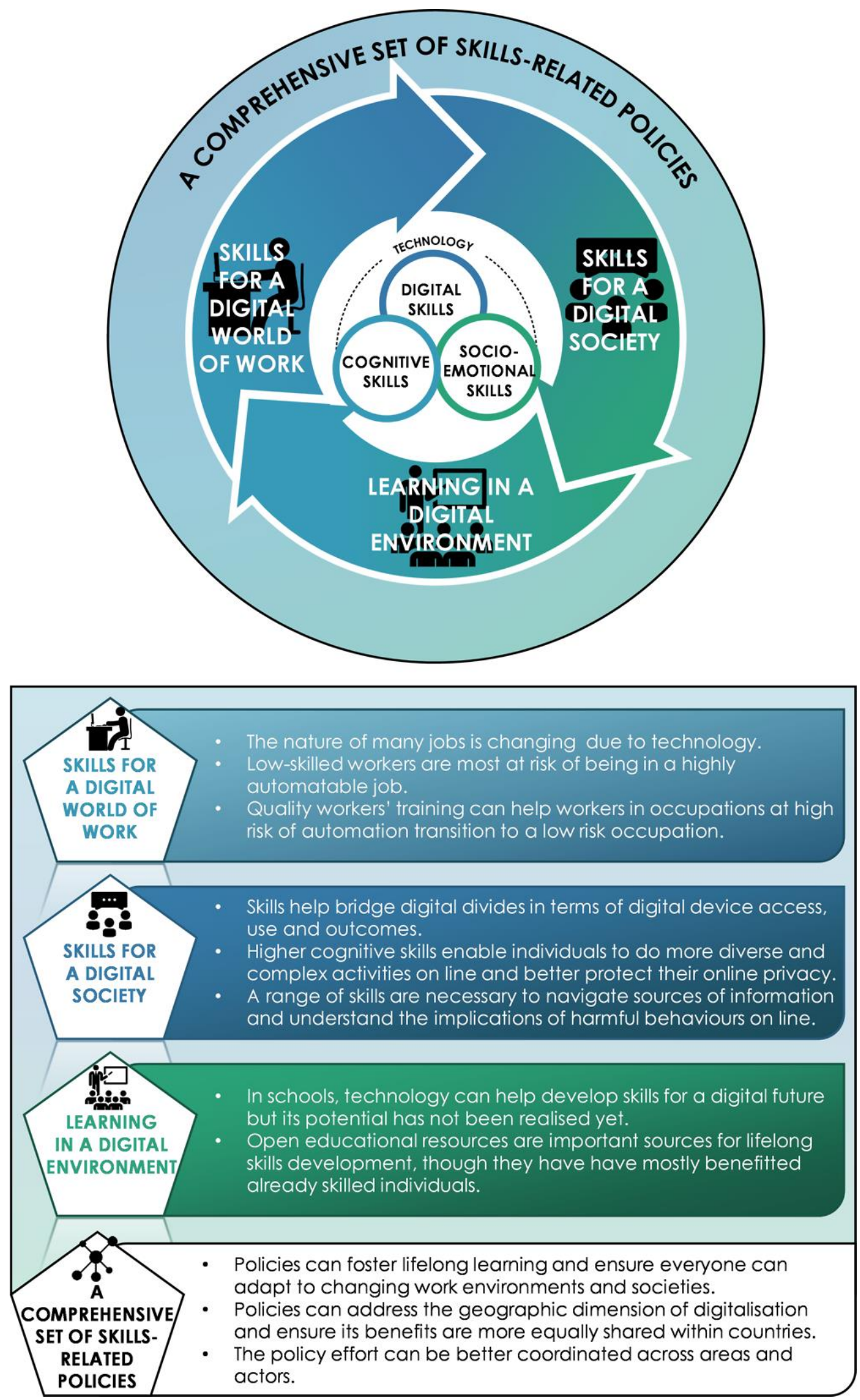

Source: OECD (2019[9]), Skills Outlook 2019: Thriving in a Digital World 


\section{Skills at the organisation and sectoral level}

Adopting a comprehensive set of skills-related policies that address needs at a societal level is valuable for supporting digital transformation in general. However, organisations and sectors need to reflect on the implications of the digital transformation for their activities and ways of working in order to take the necessary steps to thrive. For the purposes of this paper, we consider this organisation and sectoral layer to refer to the experience of the public sector across all tiers of government.

Public servants need to deliver public value while addressing complex challenges and adapting to changes in how the public sector operates. The OECD has developed a framework identifying the necessary skills for a high-performing civil service that generates public value (Figure 2.5) (OECD, 2017 $[12]$ ). The activity of civil servants is categorised into 4 areas of interaction, each requiring professional, strategic, and innovation skills:

- with elected officials, requiring policy advice and analytical skills,

- directly with citizens, requiring service delivery and citizen engagement skills,

- in collaboration with a range of stakeholders, requiring network management skills, and

- through non-governmental delivery systems, requiring commissioning and contracting skills,

Figure 2.5. Civil service skills for public value: a framework

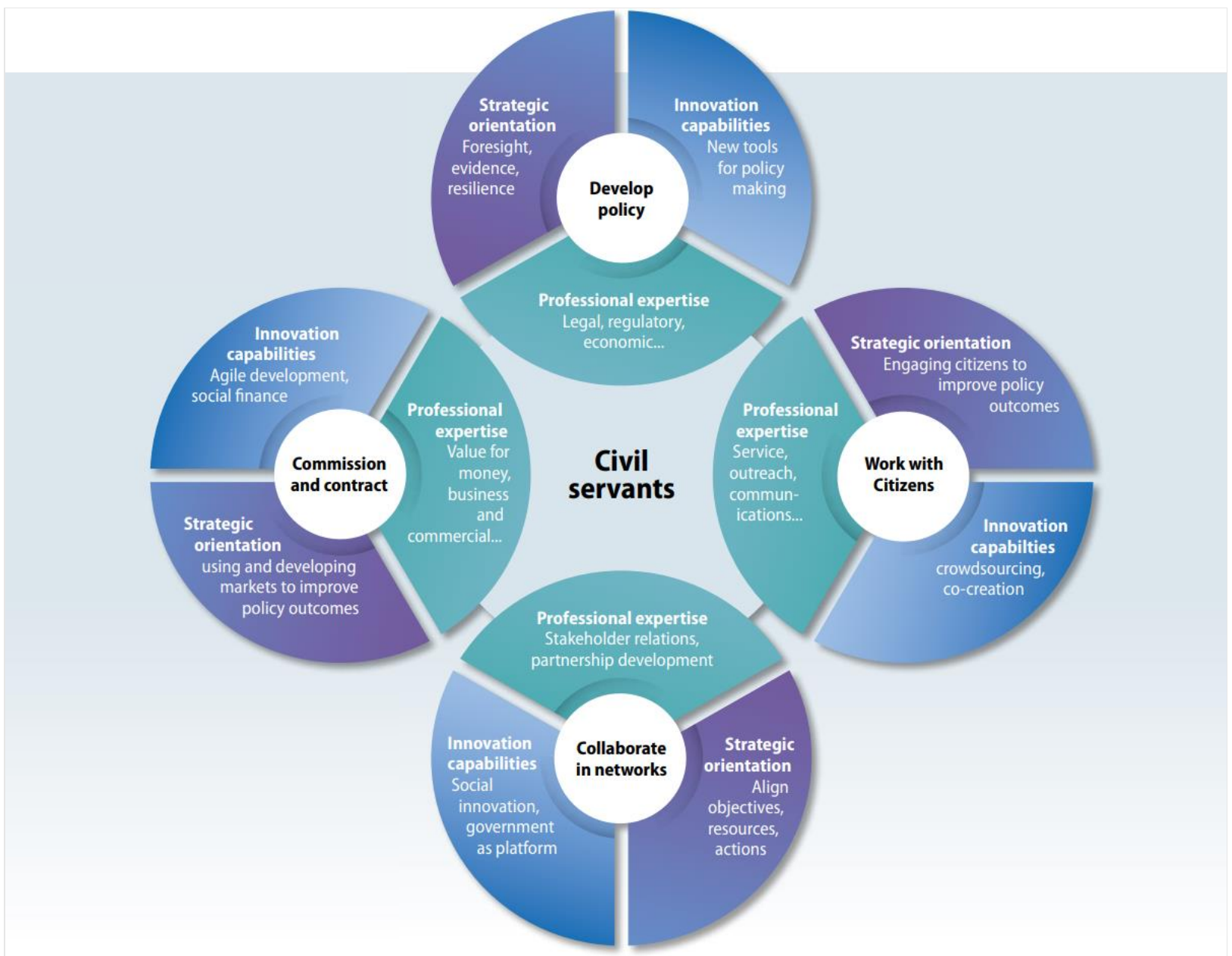

Source: OECD, $\left(2017_{[13]}\right)$, Skills for a High Performing Civil Service. 
The framework focuses on the skills needed in each area and proposes practices based on country examples as ways to fill the gap. These include investing in data-driven and user-driven approaches, making the public sector an attractive employer, supporting an effective learning culture and building networks to apply skills $\left(\text { OECD, } 2017_{[13]}\right)^{3}$. These practices and this framework are of critical importance for creating the overarching environment in which more detailed instruments can be developed to guide topic or profession specific policies at the level of individuals or teams.

\section{Skills at the individual and team level}

Translating the opportunities of the digital age at an organisation and sectoral level is important for renewing expectations about the nature of work. Once the general skills policies and activities have been identified for the public sector, then there is a need to develop policies at the individual and team level that focus on the skills needs of public servants both in terms of their day-to-day roles as well as their wider competencies. These individual and team level competencies should form part of organisational skills approaches in order to acknowledge and adopt cross-cutting lessons and benefits more widely.

One example of this is the work of the OECD's Observatory of Public Sector Innovation on core skills for public sector innovation (OECD, 2017 $\left.{ }_{[12]}\right)$. The six skills areas in Figure 2.6 are designed to show public organisations the skills that are needed to embed innovation capabilities in general and to develop the capabilities of those employed in roles focused on innovation. Before talking about innovation, there is a need to create innovators in the public sector. As a result, the framework implies that innovation comes from a workforce with the skills to think and behave innovatively. Not all public servants need to practice those skills daily but there should be some level of awareness in each competency.

\section{Figure 2.6. Six skills areas for public sector innovation}

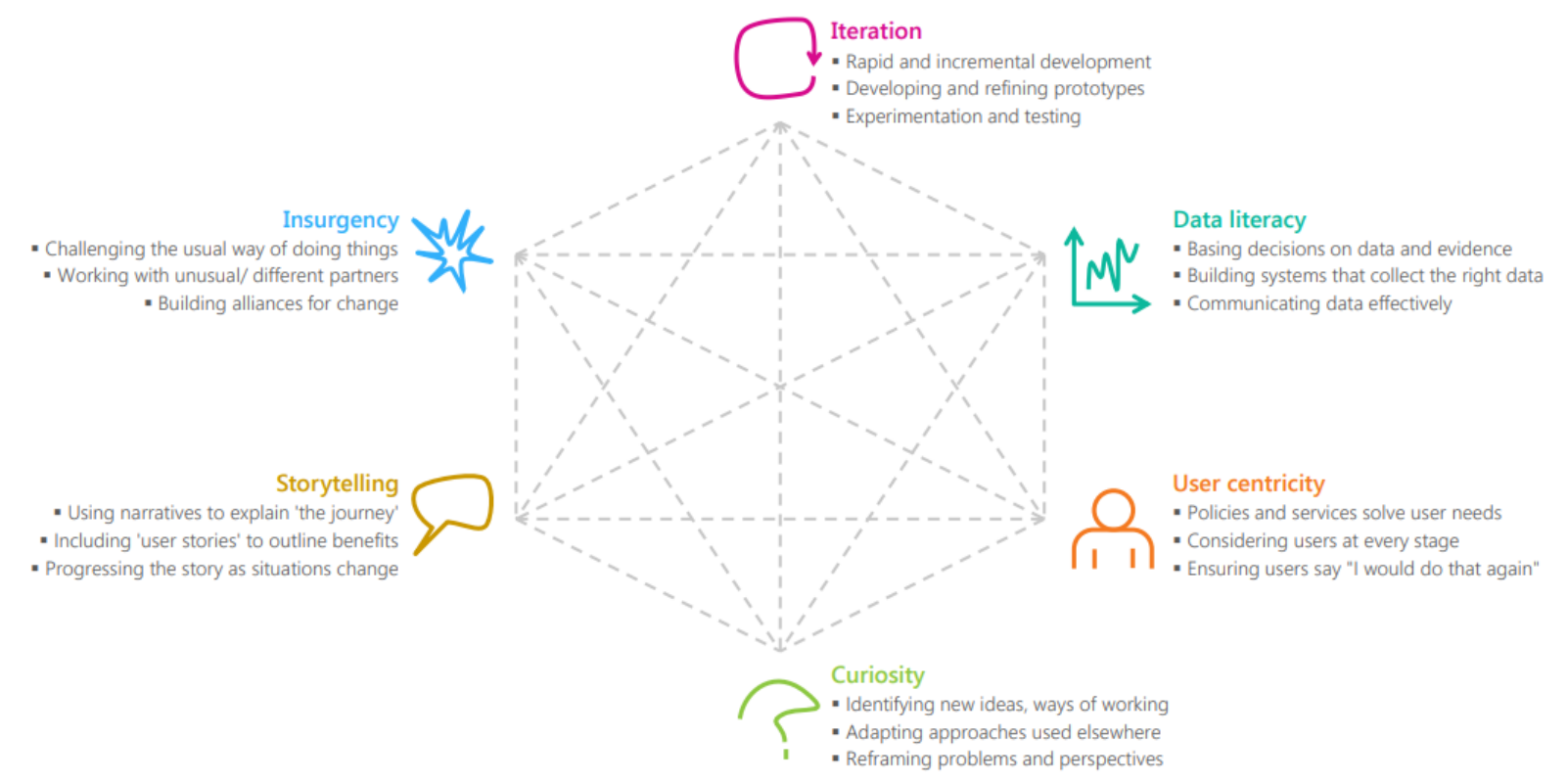

Source: OECD (2017 $[12])$, Core skills for Public Sector Innovation

\footnotetext{
${ }^{3}$ For a fuller discussion on establishing data-driven and user-driven public sector capability for effective, empirical, decision making see The OECD Digital Government Policy Framework: Six dimensions of a Digital Government (OECD, 2020[5]), The Path to Becoming a Data-Driven Public Sector (OECD, 2019 ${ }_{[58]}$ ) or Digital Government in Chile - Improving Public Service Design and Delivery (OECD, 2020[27])
} 
A second individual and team level perspective with relevance for digital government is found in discussions on the theme of public sector leadership. The leadership framework for a high-performing civil service (Figure 2.7) recognises four capabilities and intends to help public sector leaders understand their performance in terms of abilities, motivations and opportunities, while assisting them to develop their own workforce. The framework recognises that leaders need to:

- exhibit values-based decision making;

- be open to the challenge of other perspectives;

- act as organisational stewards by reinforcing trust and ensuring the workforce has tools and skills needed to carry on their tasks, and

- create a network of collaboration with internal or external stakeholders (Gerson, 2020[14]).

Figure 2.7. Four leadership capabilities

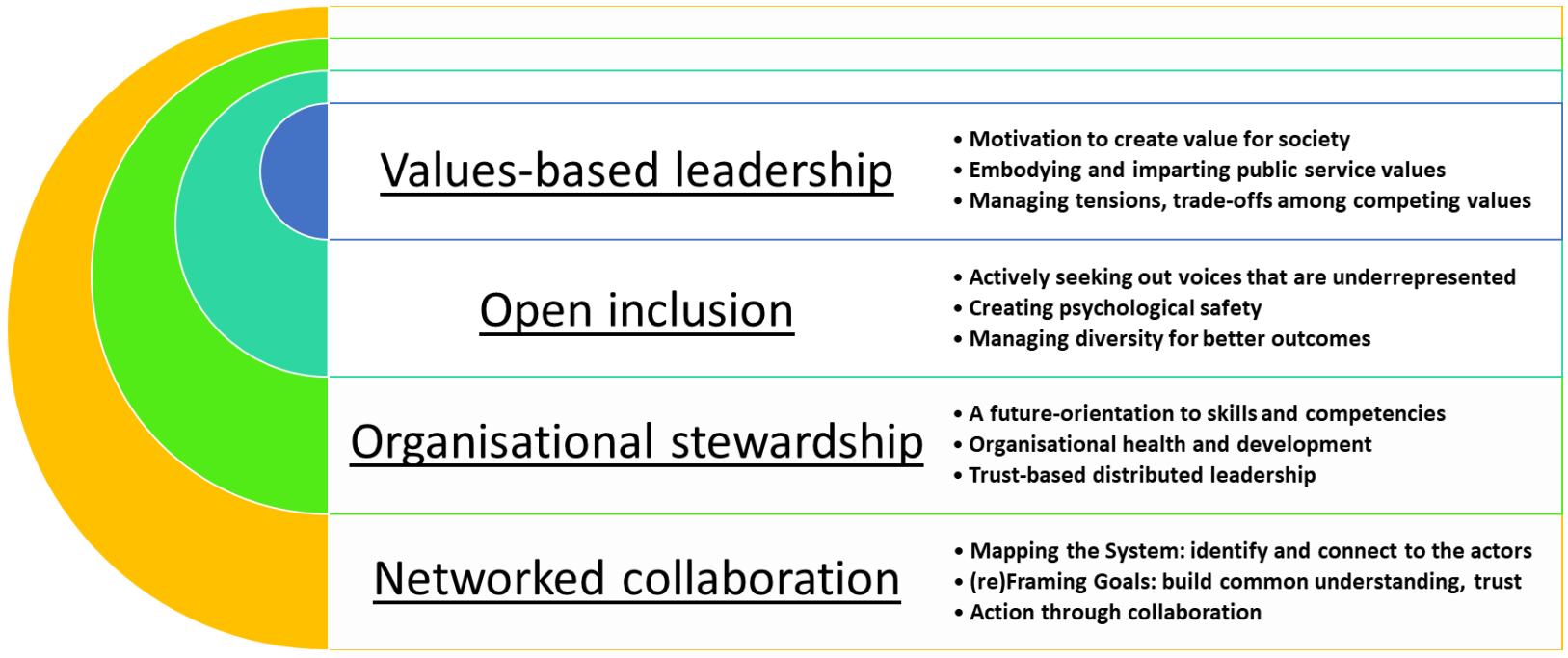

Source: Gerson (2020[14]]), Leadership for a High Performing Civil Service: Towards senior civil service systems in OECD countries

\section{The OECD Framework for Digital Talent and Skills in the public sector}

The framework advanced by this paper contributes to the discussion around skills at the individual and team level but draws on, and contributes to, an expectation that coherent policy efforts are embedding the talent and skills for digital transformation throughout the public sector and society in general.

The framework reflects the evolution of thinking by the OECD around the role of talent and skills in establishing digital government maturity. A preliminary model was first elaborated in a study of the digital transformation of African Portuguese-Speaking countries and Timor-Leste (OECD, 2018[15]), and then further developed in the Digital Government Reviews of Brazil and Panama (OECD, 2018[16]; OECD, 2019 $\left.{ }_{[17]}\right)$. The OECD Framework for Digital Talent and Skills in the public sector (Figure 2.8) iterates this earlier work to develop the conversation further and is structured around three pillars:

1. The context for those working on digital government and the environment required to encourage digital transformation.

2. The required skills to support digital government maturity, covering all public servants, in particular professionals and those in leadership roles.

3. The practical steps and enabling activities required to establish and maintain a workforce that encompasses the skills to support digital government maturity. 
Figure 2.8. The OECD Framework for Digital Talent and Skills in the Public Sector
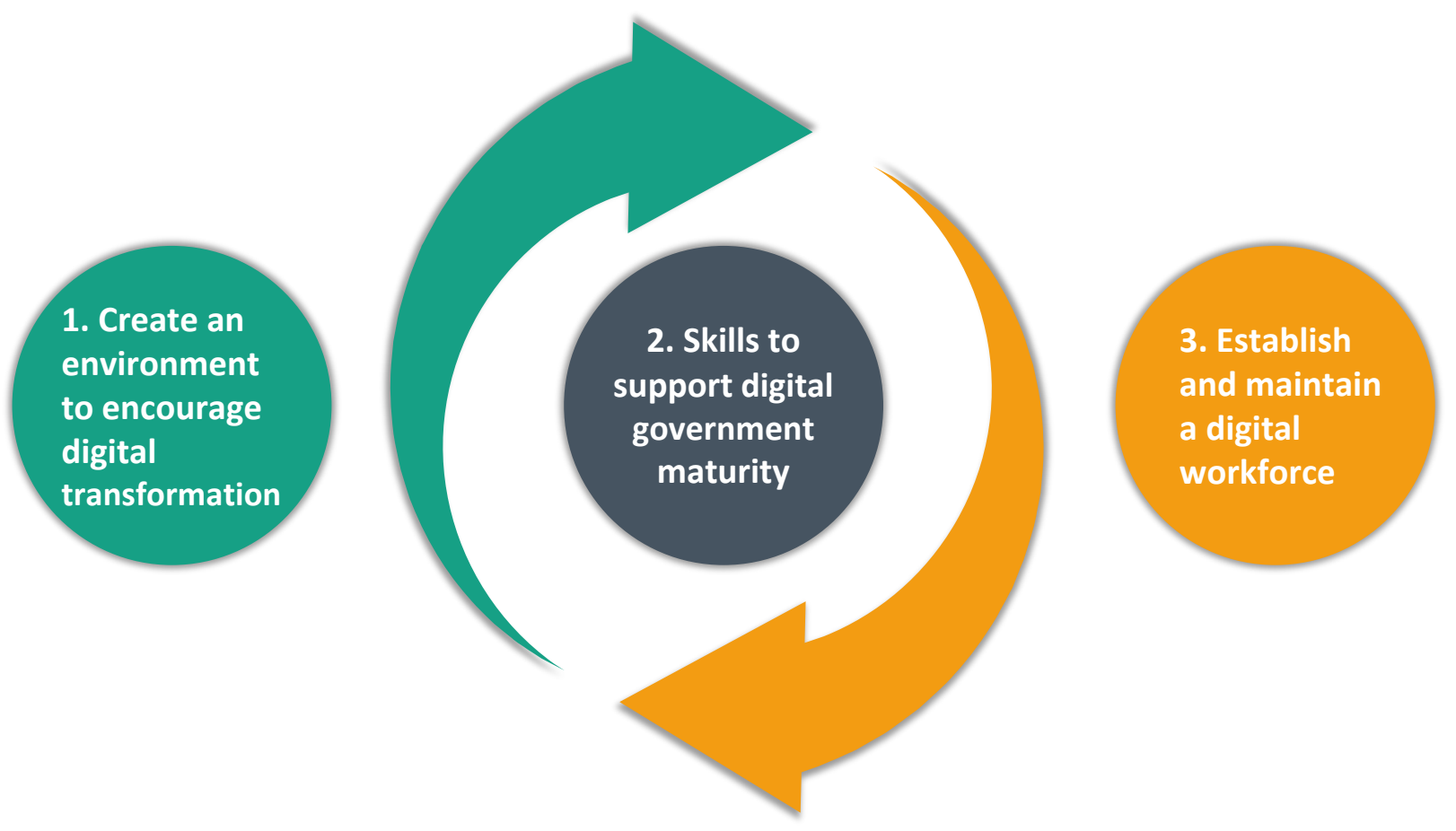

Source: Authors' own design

Individual- and team-level skills provide the raw material for digital government maturity (Pillar 2), but whether digital government efforts flourish is determined by the relationship between the work environment (Pillar 1) and ongoing efforts to establish and maintain a digital workforce (Pillar 3 ). The paper considers each of these pillars in turn before concluding with recommendations for governments to consider as they seek to develop greater digital government maturity.

Chapter 2 considers the environment for encouraging digital transformation (Pillar 1) and will help governments appraise their leadership, organisational structures, learning culture, and ways of working. By the end of Chapter 2, readers will understand how conducive the workplace environment is to a flourishing digital workforce from a cultural, leadership, and organisational point of view.

Chapter 3 defines the skills to support digital government maturity (Pillar 2). Within the broader context of $21^{\text {st }}$ century skills in society, four areas of skills to support digital government are presented: user skills, socio-emotional skills, professional skills and leadership skills. By the end of Chapter 3, readers should be able to identify skills and competencies their organisations need to develop in order to strengthen their digital government maturity.

Chapter 4 considers the practical steps necessary to attract, recruit, retain and develop a workforce for achieving sustainable digital government maturity (Pillar 3). The section looks at recruitment methods, career planning, workplace mentoring, training and the role of the private sector. By the end of Chapter 4 , readers will be able to identify opportunities to improve their approach to the different areas to ensure that the workforce is, and remains, sufficiently digital. 


\section{The right environment to encourage digital transformation}

Creating an environment that allows and facilitates the development of skills and talents to encourage digital transformation is not a unique challenge for the public sector. The introduction discussed how the OECD Skills for a Digital World framework identifies learning in a digital environment as a fundamental component of equipping the world for the digital era (OECD, 2019 $\left.{ }_{[9]}\right)$. Furthermore, at the organisational and sectoral level of the public sector itself, the OECD Skills for High Performing Public Service framework (Figure 2.5) recognises leadership, learning culture and building networks to apply skills as essential for developing a highly skilled public sector (OECD, 2017[13]). Building on this previous work, this chapter explores the first pillar of the OECD Framework for Digital Talent and Skills in the Public Sector (Figure 3.1) and argues that the combination of technology, a skilled workforce and the right work environment underpins complete transformation. If done well, the transition to a digital workplace will empower and encourage public servants to take initiative and lead the change to a digital culture, which in the long run, will nurture relationships with citizens and deliver better public services.

Figure 3.1. The OECD Framework for Digital Talent and Skills in the Public Sector (focus on Pillar 1)
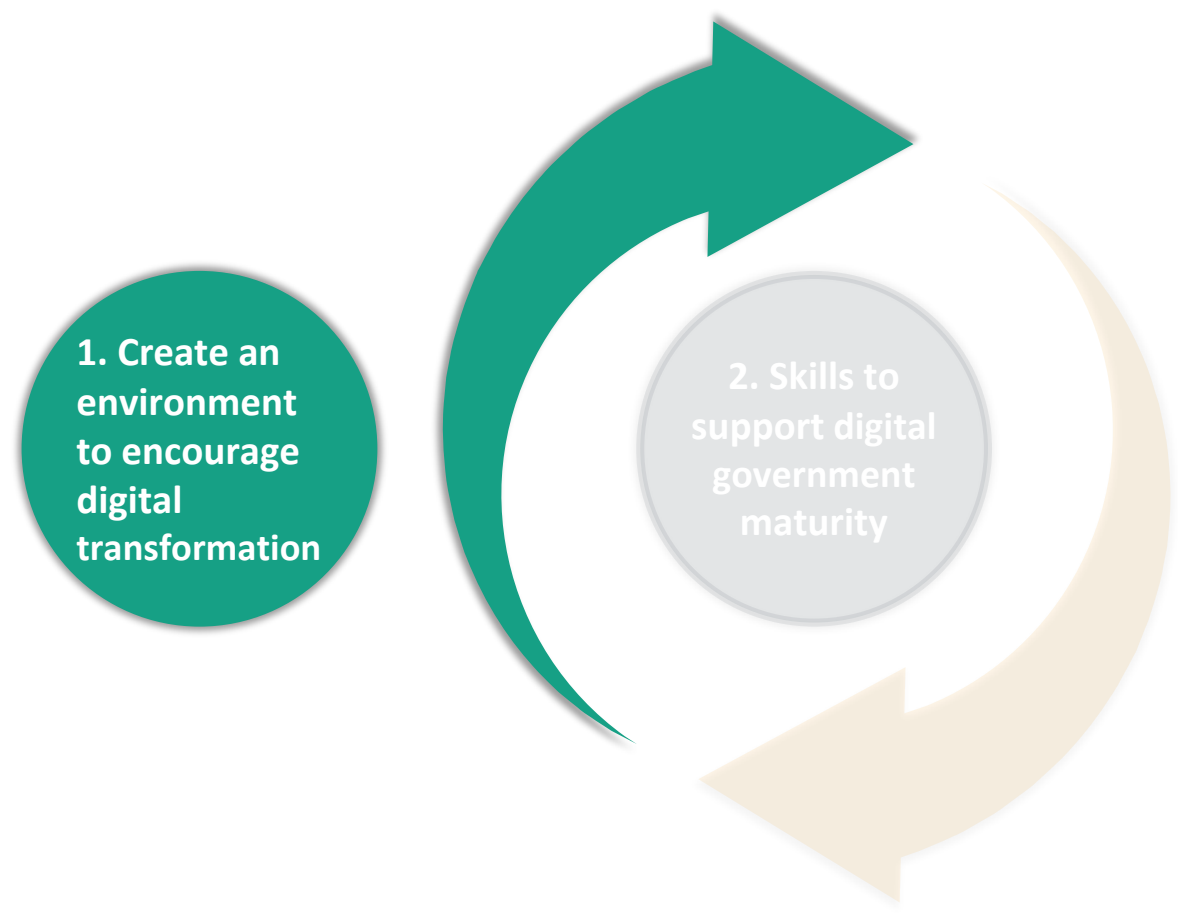

Source: Authors' own design 
Public sector organisations exist to meet the needs of the economy and society. This sense of purpose can be a powerful attraction and motivator for people choosing to commit their professional lives to public service. This benefit extends to giving public sector organisations an enthusiasm for evolving their operating environments and working practices to improve outcomes for all. In this way, digital government approaches that put citizens at the centre and increase the proximity between public servants and citizen needs are welcome from both an organisational, and individual, perspective. As countries embrace the paradigm shift from e-government to digital government, they are creating new practices, exploring new ideas and embracing innovative activities to understand the needs of society and better meet citizen needs enabled by digital technology and data, while learning and improving over time.

To effectively use data and technology to respond to the needs of the economy and society, public sector organisations need a capable workforce that is empowered to work across organisational silos and equipped to place the needs of people at the core of policy making processes. Achieving these outcomes relies on teams working to address governance issues around collaboration or technical and regulatory issues concerning data access and sharing. Therefore, policies need to focus on creating an environment in which diverse and multi-disciplinary teams can thrive such as through embracing more flexible ways of working (see Box 3.1). Thriving teams can stimulate individual performance by encouraging a sense of belonging in the workplace. Engendering the feeling of acceptance within a group of people can help staff to feel included and safe to be themselves. Conversely, if an employee does not feel part of a team, motivation and productivity can be diminished, and both performance and wellbeing can suffer.

\section{Box 3.1. Emergent approaches to delivery}

Traditional models of public service project delivery have favoured sequential approaches that try to manage certainty through gathering requirements upfront and formal decision points. Rapidly changing environments and a need to account for the evolving long-term reality for products and services means organisations are reviewing their approaches to funding, commissioning and delivering public services. Among the approaches being explored amongst the OECD Working Party of Senior Digital Government Officials (E-Leaders) are Lean, Agile and Scrum.

\section{Lean methodology}

Lean methodology is a way of creating the maximum value for customers with the minimum resources (Lean Enterprise Institute, 2020[18]). This means optimising people, resources, effort, and energy of an organisation toward creating value for the customer by continuous improvement and respect for people.

\section{Agile methodology}

The authors of the Agile Manifesto chose "Agile" because the word represents adaptiveness and response to change. The concept of Agile is about thinking through how to understand today's environment, identify uncertainty, and figure out how to adapt to that. (Agile Alliance, 2020[19])

\section{Scrum framework}

Scrum is a framework for team collaboration to address complex adaptive problems, while delivering products of the highest possible value. It implements the scientific method of empiricism and replaces a programmed algorithmic approach with a heuristic one, with respect for people and self-organisation to deal with unpredictability and solving complex problems (Scrum.org, 2020[20]).

This chapter will look at the contextual elements that can empower teams and improve their capability to lead digital transformation including, leadership, organisational structure, learning culture and ways of working. When taken together, these individual priorities can transform a workplace into the right environment to receive a digital workforce and fully implement digital government strategies. 
First, leadership. It is fundamental for there to be the vision to guide an organisation's shift from egovernment to digital government and in so doing, nurture a culture of experimentation, curiosity and learning from failure. Effective leadership will provide the organisation with clear digital strategies and support the design of a work environment reflecting the norms and values of their leadership.

Organisational structure plays another important role since the creation of a flatter hierarchy, communities of practices and robust networks across the entire organisation increase the quality of communication, exchange and advancement of skills (Kubheka, Kholopane and Mbohwa, 2013[21]). Rewriting job descriptions and reshaping job families can also help to consider the implications of the digital transformation on roles and the nature of delivery. This activity supports a wider engagement with skills at the societal level in terms of workforce planning not only in the public sector to understand any shortage of professionals with the right digital skills and direct efforts to shape education and employment policy to equip the wider labour market with the suitable skills for greater employability and economic growth. This will build up public servants' satisfaction, contribute to a healthy digital workplace and influence public servants' productivity, motivation and happiness at work.

A digital environment must also develop a life-long learning culture. Formal training and models of informal support (further developed in Chapter 4) encourage public servants to advance in their skills and career path while keeping them motivated and open to "learning to learn" (OECD, 2019 [22]), which facilitates the adoption of new ways of working (OECD, 2014 $\left.4_{[23]}\right)$. Indeed, ways of working should evolve in parallel with the environment. Being equipped with the necessary digital technologies, and adopting agile methodologies contribute to making a workplace digitally enabled.

\section{Leadership}

\section{A leadership that creates an environment to encourage digital transformation will communicate a clear vision for digital government, and actively champion its benefits. Leaders will be engaged, visible and approachable, and empower their teams through decentralising decision making}

Fundamental to achieving sustainable digital government efforts and maintaining momentum for digital government maturity is political, organisational and societal leadership. Leadership is often defined as the process by which an individual influences and guides a person or a group of people to achieve a common goal (Silva, 2016[24]) (Winston and Patterson, 2006[25]). From the perspective of political leadership, this includes having a clearly expressed vision for the role of digital in the future of the country and as an extension, the implications for service design and delivery. While elected representatives set the political direction and high-level vision, design and delivery of services belongs to the civil service. That leadership needs to ensure internal alignment, working closely with leaders across the public sector to embed the importance of designing and delivering high quality services in the day-to-day work of the civil service as a whole (Kulkarni and Scott, $\left.2019_{[26]}\right)$. Finally, there is an important leadership role provided by those who are neither elected by the public, nor employed by government with the external voice from civil society, academia and the private sector (OECD, 2020[27]).

The OECD Recommendation on Public Service Leadership and Capability (OECD, 2019[28]) outlines how countries can ensure that their public sector workforces are fit for purpose for today's policy challenges and capable of taking the public sector into the future. Its principles set the agenda for public service leaders to build a values-driven culture, encourage skilled and effective public servants and enable responsive and adaptive public employment systems. This includes an emphasis on the principles of 
innovation, data-driven decision-making, and organisational stewardship to design tomorrow's public service organisations and their workforces.

In the context of that recommendation there should be an expectation on those in leadership to embrace digital government and develop a strong understanding of its core principles through technology and data expertise (Kulkarni and Scott, 2019[26]) (see Digital Leadership skills in Chapter 3). The role of senior leaders as champions for increased digital maturity and promoting a digital culture and mindset is increasingly recognised as a requirement by senior digital government officials from OECD member and non-member countries. Although digital leadership is important across public service, a 2020 survey conducted by the OECD's Public Employment and Management working party found that this is still among the least mentioned items in leadership competency frameworks. For example, in France, the public service looked at the challenge of embedding digital leadership and realised that senior leaders only attend trainings which are usually short and therefore superficial. To solve this issue, the French Digital Directorate (DINSIC) ${ }^{4}$ established a coaching programme with digital professionals to accompany senior leaders (Gerson, 2020[14]). Senior leaders and managers have no choice but to move past traditional notions of public service leadership based on legal compliance or process management in order to properly embrace and lead the digital transformation. Indeed, it is imperative that senior leaders do not abdicate responsibility for developing the necessary skills to nurture, encourage and champion digital government.

A part of leadership involves decision making. Traditionally decision making was concentrated at the top of an organisation in the hands of a few according to a hierarchy and did not delegate authority to others to make product level decisions. However, digital leadership favours the distribution of authority and autonomy throughout an organisation, allowing decision making to be taken in greater proximity in both time and delivery to the work at hand without introducing layers of gate keepers. Moving away from centralised decision making can help to increase flexible working by encouraging flatter organisational structures that empower teams to make their own decisions on a de-centralised basis.

The COVID-19 pandemic has highlighted the extent to which the capacity to respond to a changing work environment shapes leadership. There will be much to reflect on in the aftermath of the COVID-19 pandemic about how to effectively lead and manage organisations but the challenges under the 'new normal' are not necessarily new. Writing for the MIT Sloan Management Review in 2016, Lynda Gratton posed the question 'within the next five years, how will technology change the practice of management in a way we have not yet witnessed?' (Gratton, 2016[29]). Her answer echoes the challenges facing leaders in responding to COVID-19 from a day-to-day operational point of view such as managing virtual or hybrid teams, handling the different technology preferences of multigenerational groups and supporting effective knowledge transfer. However, the COVID-19 response has also brought opportunities to reflect on some of the lessons learned such as investing in digital professions and planning capability development trainings to make governments more flexible during crisis.

Since research recognises that interactions between leaders and the workforce are one of the biggest contributors to productivity, this process is extremely powerful (UK Government Communication Service, $2020[30]$ ). In large organisations, leaders can be elusive figureheads who are not always visible or accessible but leaders cannot simply set the vision, they must also act as role models. Leaders need to keep the workforce informed about where the organisation is heading, an activity that increases their accountability and creates an environment that demonstrates commitment to their position and their staff. Studies show that hearing information from leaders makes a difference to the engagement levels of a workforce by helping leaders appear more human, more visible and more caring (Gallup, 2016[31]). Instead of relying on a model of reporting back where leaders take a more passive role, leadership of digital

\footnotetext{
4 France's Digital Directorate «Direction interministérielle du numérique et du système d'information et de communication de l'État » (DINSIC) changed into « Direction interministérielle du numérique » (DINUM) in October 2019.
} 
government is strengthened by those who participate in meetings and attend internal activities to communicate more effectively and interactively with their workforce. This contributes to building a work environment with high level of reliability, visibility and trust within the organisation.

Moreover, leaders who find ways to operate in greater proximity to their workforce can identify needs and challenges faster, and are able to provide timely support and funding. Practices like regular Show and Tell - meetings that not only enable teams to showcase projects they are working on and lessons learned, but also give credit to their work, which empowers and motivates them to do better - can create an opportunity for leaders to get closer to their staff, listen and react to current work. In 2014, the United Kingdom's Department of Health and Social Care held their first Digital Show and Tell to showcase recent digital projects and explain how they run them (Stansfield, 2015[32] $)$ It is essential for leaders to have regular interactions with their workforce, so that they can understand and observe the issues their staff face to better intervene strategically and unlock funding when needed.

\section{Table 3.1. Leadership for creating an environment to encourage digital transformation}

\begin{tabular}{l|l}
\hline What supports digital transformation & What doesn't support digital transformation \\
\hline Communicate a clear vison of the role of digital & Unclear vision of the role of digital \\
\hline Actively champion the benefits of digital government & $\begin{array}{l}\text { Little demonstrable knowledge or interest in the benefits of } \\
\text { digital government }\end{array}$ \\
\hline Decentralised decision making & Centralised decision making \\
\hline Actively engaged, visible and approachable leader & Aloof, figurehead leader \\
\hline
\end{tabular}

\section{Organisational structure}

\section{An organisational structure that creates an environment to encourage digital transformation will reflect flatter hierarchies and distributed decision making with job families, roles and descriptions focused on meeting user needs rather than implementing technology.}

An organisational structure is the framework of an entity, as the bones are to the skeleton of a human body. It structures how operational activities are distributed, organised and managed as well as how the components relate to each other (Ahmadya, Mehrpourb and Nikooraveshb, 2016 ${ }_{[33]}$ ). This enables the workforce to understand who manages what processes and learn about internal dynamics of power, responsibility and decision-making delegation (Hodge, Anthony and Gales, $1991_{\text {[34]). The structure plays }}$ a crucial role in setting the right environment for digital transformation as organisations whose structures make "learning" core to their value, vision and functions have a positive impact on the attitudes and productivity of their workforce (Kanten, Kanten and Gurlekc, 2014[35]).

Conversely, the structure of an organisation and its employment models can limit the development of a digital workforce, the creation of a learning environment and the prioritisation of a more flexible and 
collaborative approach to work. Furthermore, Conway's Law ${ }^{5}$ (Conway, 1967[36]) recognises the inadvertent repercussions of organisational structure in the design of the systems they deliver, a particularly frequent issue for public services that end up reflecting the structure of the organisations they are built by (MacCormack, Rusnak and Baldwin, 2008[37]). This was experienced by the Digital Transformation Office (DTO) in Australia in 2015 when they identified 1027 government websites which were found to be aligned to the siloed structure of government agencies with little connection between the underlying digital services. With a mandate to provide services that meet the needs of citizens, rather than reflecting the structure of government, the DTO established the role of 'Service Managers', a senior executive with responsibility for the "whole end-to-end user experience" of services across and between government organisations. Structuring public sector teams and their leadership to reflect user journeys can be an effective way to counter the effects of Conway's Law.

A flat or organic structure is considered to be better suited to more flexible and suitable for digital transformation than hierarchical ones (Kubheka, Kholopane and Mbohwa, 2013[21]). Hierarchical models are more bureaucratic and traditionally take a top-down approach to establishing and approving processes, decision-making and ways of working. By contrast, flat structures distribute decision-making within the team responsible for a task as well as encouraging a wide-span of control with several teams answering to one person. This can allow flexibility in fast-moving environments and empower a workforce to experiment and take risks. Minimising the organisational distance from leaders can help staff feel their leaders are more accessible and thereby reduce hierarchy-related pressures. Flat structures are not without their challenges. For example, when teams disagree, it might be difficult to align decisions, making it important to design a means of establishing consensus. Furthermore, initiating such a structure can be difficult because it requires those with power to unilaterally give it away unless encouraged to do so by an external process such as the one described in Box 3.2.

\section{Box 3.2. The Australian Public Service Review}

In May 2018 the Australian Government commissioned a review to ensure the Australian Public Service (APS) was fit for purpose. The process engaged with more than 11000 individuals and organisations and over 400 consultations to conclude that service-wide transformation was needed to achieve better outcomes. This was not say that the APS was broken but that the status quo was insufficient to prepare for the changes and challenges anticipated in the next decade. Recommendation 32 was to:

\section{Streamline management and adopt best-practice ways of working to reduce hierarchy, improve decision- making, and bring the right APS expertise and resources.}

The implementation guidance called for management structures to have no more organisational layers than necessary in order to allow for decision-making at the lowest practical level with spans of control reflecting the type of work being managed, structures providing flexibility to respond to changes, and jobs classified according to work level.

Source: Commonwealth Government of Australia, $\left(2019_{[38]}\right)$. Our Public Service, Our Future. Independent Review of the Australian Public Service.

The classification of job families and their associated job descriptions is a further factor in the organisational structure for encouraging digital transformation by determining the profiles and skills to support delivering on an organisation's vision. Job families are grouping of jobs with similar vocations, skills and

\footnotetext{
5 "any organisation that designs a system (defined broadly) will produce a design whose structure is a copy of the organisation's communication structure."
} 
competencies, which can determine career path, pay characteristics and conditions (Reuilly, 2004[39]) (further developed in Chapter 4). As such they need to be clearly arranged to facilitate the description of general tasks, skills and responsibilities a position requires to fulfil its mission and enable public sector digital transformation.

The public sector's purpose of serving society remains constant but the same is not true for the means by which this is done. The digital transformation has elevated citizen expectations of services and made it possible to move away from putting narrow transactional exchanges online towards the delivery of services understood from when someone first attempts to solve a problem through to its resolution (from end to end); on a continuum between the user's experience and processes for back-office staff (external to internal); and across any and all of the channels involved (omni-channel) (OECD, 2020[27]). To design and deliver services in the digital age it is therefore necessary to revise any existing roles and capabilities aligned with earlier ideas of e-government. While these Information and Communications Technology (ICT) professions require the capability to navigate a "technology-rich environment" (OECD, IDB, 2016[40]), digital professions are informed by the role of technology in service to prioritising users' needs. As explained by the Digital Technologies hub, "ICT is knowing how to play a game whereas Digital Technologies is about "creating' the game people play" (2020[41]).

In other words, the job families and descriptions required to support digital government do not start from the premise of a particular technology but view technology as a secondary enabler, allowing for a focus on meeting the need of a user through re-engineering and re-designing the services available to them. This makes it fundamental to set the expectation of considering the user experience of navigating policies or services as a core idea of digital government maturity.

Therefore, job families need to be revised to reflect an approach that is more user-centric, iterative and collaborative compared to traditional approaches which tend to operate independently without collaboration or alignment. Organisations also need to be flexible enough to create new job profiles when deemed necessary. This can be supported by preparing job descriptions that focus on objectives and outcomes rather than detailing discrete tasks. In the United Kingdom, the development of the Digital, Data and Technology (DDaT) Profession Capability Framework has formally recognised a series of job families needed to effectively digital, data and technology projects and improve consistency in both job roles and the definition of the associated skills, with the example of Service Designer discussed in Box 3.3.

\section{Table 3.2. Organisational structures for creating an environment to encourage digital} transformation

\begin{tabular}{l|l}
\hline What supports digital transformation & What doesn't support digital transformation \\
\hline Reduce organisational layers & Hierarchical organisation \\
\hline User-centric, iterative and collaborative job families & No collaboration/alignment of job families \\
\hline Job descriptions focused on objectives and roles & Job descriptions focused on discrete tasks \\
\hline Focus on digital professions & Focus on ICT professions \\
\hline
\end{tabular}




\section{Box 3.3. United Kingdom DDaT Profession Capability Framework: Service designer}

The Capability Framework describes the service designer profession, including:

- introduction to the role and communicating what it involves and the skills it requires

- a description of the career path from associate service designer to head of service design, specifying the skills needed for each level

\section{Example of Associate service designer job description:}

As a trainee in an entry-level position, working under supervision, you will need design aptitude, potential and an understanding of the role. Skills needed for this role:

- Agile working. You know about agile methodology and the ways you can apply the principles in practice. You can take an open-minded approach; you know why iteration is important and can do it quickly. (Relevant skill level: awareness)

- Communication skills. You know about the need to translate technical concepts into nontechnical language and understand what communication is required for internal and external stakeholders. (Relevant skill level: awareness)

- Community collaboration. You understand the work of others and the importance of team dynamics, collaboration and feedback. (Relevant skill level: awareness)

- Digital perspective. You demonstrate an awareness of design, technology and data principles. You are engaged with trends in design and know how to set priorities. You understand the internet and the range of available technology choices. (Relevant skill level: awareness)

- Evidence- and context-based design. You know about the value of evidence-based design and that design is a process. (Relevant skill level: awareness)

- Facilitating decisions and risks. You identify technical disputes and describe them in ways that are relevant both to direct peers and to local stakeholders. You can work collaboratively while recommending decisions and the reasoning behind them. (Relevant skill level: awareness)

- Leadership and guidance. You are committed to agreed good practice for the team, teaching new starters and challenging substandard work by peers. You can recommend decisions and describe the reasoning behind these. You can identify and articulate technical disputes between direct peers and local stakeholders. You know about the importance of team dynamics and collaboration. You understand the importance of feedback. (Relevant skill level: awareness)

- Prototyping in code. You have a basic knowledge of how the internet works. You can use tools and change text. You can edit existing code and 're-use' it. (Relevant skill level: awareness)

- Prototyping. You know about prototyping and can explain why and when to use it. You know how to work in an open and collaborative environment- for example, by pair-working. (Relevant skill level: awareness)

- Strategic thinking. You know about the strategic context of your work and why it is important. You support strategic planning in an administrative capacity. (Relevant skill level: awareness)

- User focus. You can identify needs and engage with users or stakeholders to collate user needs evidence. You understand and can define research that fits user needs. You can use quantitative and qualitative data about users to turn user focus into outcomes. (Relevant skill level: working)

Source: Government Digital Service (2020[42]), Service Designer 


\title{
Learning culture
}

\section{A learning culture that creates an environment to encourage digital transformation must be embraced at all levels of an organisation but starts from an organisation's leadership in encouraging and providing a safe environment in which employees can experiment, learn and develop through testing, iterating and failing}

\begin{abstract}
Given the rapid evolution of digital technology, digital skills need to be able to mature and respond over time. This means creating environments where staff are encouraged to continuously learn and grow. One of the responsibilities of digital leaders is to encourage the establishment of such environment and support learning initiatives. Although this sounds natural, creating such a learning culture requires leaders to not only promote a safe environment where employees are free and empowered to experiment with no judgment but also lead by example by working on their own development, motivate managers to help support this mindset and spread good practice.
\end{abstract}

For a successful digital transformation, it is necessary to create an empowering environment. This can be achieved through recognising and showing appreciation for the work people do and their contribution to the organisation but is also fostered through encouraging an experimental culture, which recognises the value of learning from testing, iterating and failing. It is a leaders' responsibility to create a safe workplace where rapid and multiple iteration is welcomed as a means for learning. Making experimentation an integral part of the culture for public sector organisations means encouraging teams to train in new skills, apply knowledge from experience and nurture curiosity. Establishing this mindset can help to mitigate the public sector's greater risk aversion when compared with the private sector (Guiso and Paiella, 2010[43]) because curiosity prevails and failure is seen as an opportunity to learn and not as a costly mistake.

To keep this culture of learning alive, organisations need to encourage and incentivise managers to enable and support their employees in taking time to learn and grow. Possible means of support that stimulate learning, experimenting and sharing can include mentoring, communities of practice, show and tells and retrospective meetings, as well as different types of trainings which will be discussed further in Chapter 4 . Mentoring programmes that cut across the organisational structure are essential to minimise the impacts of silos and reducing any gaps created by hierarchy. If done well, mentoring and reverse mentoring should be beneficial to both mentor and mentee. It should not be a one way teaching from the mentor, but should create mutual learning opportunities from one another. Communities of practice also support learning among groups. Participants get together and discuss a topic or a challenge in depth to try to solve it.

Besides this, such an experimentation culture should be seen as an opportunity for all public servants to feel empowered to speak out and be heard. Although it is important to recognise that there are those who will be disengaged, not feel the need to change, or acknowledge the importance of lifelong learning, leaders need to promote inclusiveness, democratise this organisational culture and give everyone equal chances to launch as well as stop experiments. This offers individuals the freedom to take new approaches they believe are relevant and at the same time, others can learn, provide feedback and improve in real time. This proves that a digital environment allows not only autonomy and collaboration within, but also beyond the organisation. The Digital Academy of Canada, for instance, (see Box 3.4) engages with several partners to broaden their learning experience. 
Table 3.3. A learning culture that creates an environment to encourage digital transformation

\begin{tabular}{l|l}
\hline What supports digital transformation & What doesn't support digital transformation \\
\hline Encourage and provide safety for employees to experiment & No incentive to take risks \\
\hline Strong learning culture set by leaders & No learning culture in the organisation \\
\hline Learning culture embraced at all level of the organisation & No incentive for managers to promote learning \\
\hline
\end{tabular}

\section{Box 3.4. Building learning cultures within governments}

\section{Canada's Digital Academy}

The Canada School of Public Service (CSPS) Digital Academy was created in 2018 with the objective to teach Canada's federal public servants the digital skills, approaches, and mindset needed to transform public services in today's digital age.

As part of its activities, the Academy brings together partners from different spheres, including government, academia and the private sector, with the focus on collaboration and the sharing of knowledge and experience. The Academy offers both general and more specialized learning opportunities, in the classroom and online, for public servants at all levels.

Source: Government of Canada (2020[44]), Digital Academy

\section{Italy's Digital Skills for the Public Administration}

"Digital Skills for the Public Administration" is an initiative promoted by the Department of Public Administration within the National Operational Programme "Governance and Institutional Capacity 2014-2020". It aims to equip all public employees with common digital skills, by implementing a structured gap detection in digital skills and targeted and effective training.

Considering the role played in supporting the digital transition of the Public Administration, the Department of Public Administration, aims to:

- establish a common base for technological and innovation knowledge and skills among public employees;

- strengthen the institutional capacity for an efficient Public Administration through training on digital skills, delivered mainly in e-learning mode and customised based on a structured and homogeneous survey of the actual training needs;

- develop digital knowledge of public employees to implement the principles of digital citizenship, eGovernment initiatives and open government;

- promote the mapping of skills in administrations at different government levels, also in order to promote more effective HR management policies.

The initiative is based on three main components:

4. The syllabus, that describes the set of knowledge and skills, organized by thematic areas and proficiency levels, which characterize the minimum set of digital skills that each public employee should have in order to be able to work easily in an increasingly digital Public Administration; 
5. The web platform, that provides tools for skills verification tests and assessment of post-training learning based on the syllabus, as well as for the selection of the most appropriate training modules to meet the knowledge requirements identified; the platform also supports administrations in planning, managing and monitoring effective skills development paths in line with their organizational needs;

6. The catalogue, that collects training modules on the competences areas described in the syllabus, aimed at filling the digital skills shortcomings detected during the self-test phase.

Source: Provided by The Working Party of Senior Digital Government Officials (E-Leaders) Thematic Group on Digital Talent and Skills

\section{Slovenian "Innovation Training in Public Administration"}

In Slovenia, the Ministry of Public Administration runs "Innovation Training in Public Administration". This training aims to change the approach to workflow, problem solving and designing better solutions through effective communication. The programme is actively changing the administrative culture to implement higher quality state functions and digital services. The programme is performed in person and remotely.

Objectives of implementing the programme are:

- raising awareness of the importance of gaining new skills and knowledge in terms of alternative ways of work to enable a more agile and efficient response to the demands of the environment;

- to acquire competence for creative tackling of challenges and designing solutions using different methods and approaches focusing on the user and

- to acquire competence in different ways of communicating (more effective presentation of ideas, results, etc.) and in managing group communication processes.

Source: Ministry of Public Administration (2020[45]), Inovativen.si

\section{United Kingdom's Government Digital Service Academy}

The GDS Academy gives public sector professionals the skills, awareness and knowledge they need to build the best possible public services. Founded by the Department for Work and Pensions in 2014, it transferred to GDS in 2017 and has trained over 10000 public servants nationwide, established a partnership with Scotland and served as a model for foreign governments. There is a particular focus on the needs of sub-national government with the United Kingdom's Ministry of Housing, Communities \& Local Government funding places for 1000 local authority staff.

Source: Provided by the Working Party of Senior Digital Government Officials (E-Leaders) Thematic Group on Digital Talent and Skills

\section{Ways of working}

Ways of working that create an environment to encourage digital transformation will reflect not only in terms of the equipment, tools and flexible working environments, either physical or virtual, but also adopt agile methodologies, fund teams rather than projects and invest in ongoing digital transformation in how organisations work. 
The change in the ways people work reflects evolution in society. New ways of working have been introduced in recent years that embrace a wide range of measures enabling flexibility in hours and place of work (Blok et al., 2011 [46]). The COVID-19 pandemic and the required social distancing measures have further forced the world to adapt to different ways of working quickly and challenged organisations to be fully remote or hybrid. This may lead to the adoption of an increasingly flexible culture as the norm.

It is important to find a way of working that fits an organisation's vision, culture and staff to achieve better results. Understanding and identifying methods of working that facilitate digital transformation can be beneficial to the development of digital skills at a workplace. Several ways of working can achieve this: agile and user-centred working methodologies, access to the right technology and tools, flexible office space as well as flexible working policies. Formalising these new ways of working, similar to the discussion of training in the Chapter 4, needs to be approached in way that supports staff to establish new behaviours and emphasises people's feeling of agency and empowerment instead of having any sense of enforced compliance. Imposing ways of working, or by contrast treating them as a passive 'good-to-have', can increase the risks that change fails to take root. Therefore, a balance must be found to support and ensure the enthusiastic adoption of ways of working that are essential for enabling digital transformation.

There has been increasing attention placed on the notion of agility in the public sector. Whether in the discussion of the system level approach from an academic point of view (Carrasco et al., 2020[47]; Turqieh, Aoun and Nasr, 2018[48]; Mergel, Ganapati and Whitford, 2020[49]), the role of agility in terms of financial management (OECD, 2015[50]) or amongst the OECD Working Party of Senior Digital Government Officials (E-Leaders) as it relates to public sector service design and delivery. The role of Agile in government is often combined with user-centred working methodologies that champion iterative approaches that actively seek the involvement of users and are expecting to learn and refine in response to what's learnt. The core values of Agile were first set out in relation to software engineering in the Agile Manifesto (Beck et al., $2001_{[51]}$ ). In today's context, working in an agile way involves "bringing people, processes, connectivity and technology, time and place together to find the most appropriate and effective way of working to carry out a particular task. It is working within guidelines (of the task) but without boundaries (of how you achieve it)" (The Agile Organisation, 2020[52]). This way of working gives staff the freedom to choose where, when and how they want to work, which often increases productivity, motivation and job satisfaction, as people feel trusted and empowered.

To accommodate such methods of working, the physical workplace environment also needs to be designed to include features such as break out areas for brainstorming and pods for retrospective meetings. However, the tools, resources and software available to staff are an another important aspect of the workplace environment that should not be neglected. Access to what people need when they need it is an important aspect of productivity, particularly in terms of having the autonomy to make decisions about what best supports their working practices (Kratzer, Leenders and Van Engelen, 2006[53]). As an example, Australia's Digital Transformation Agency Office has a workspace that is open planned, with many collaborative work spaces and meeting rooms. They, and many other countries, have found that as a result of the COVID-19 crisis there was first a dramatic increase and then sustained use of tools that support remote meetings and virtual collaboration. This facilitates not only collaboration, innovation and interactions between colleagues, but also inclusivity of new hires.

Flexible working policies address individual needs, which are only possible if a supportive leadership is in place (see section above on Leadership). Giving staff choice about their working hours and locations, for example starting work early and finishing early, or opting to work mostly from home rather than the office, can encourage a better work-life balance and thereby enhance well-being and productivity. Flexible working policies were essential when the COVID-19 pandemic forced the wholesale closure of offices. The transition to all staff working from home was challenging in those countries where public service agencies had not developed a flexible culture towards work. In these cases, flexible measures had to be rapidly put in place and adopted. The pandemic accelerated the implementation of flexible working policies in countries such as Ireland, Slovenia, Spain and Uruguay, bringing forward remote working practices, 
30 | GOV/PGC/EGOV(2020)9

including the use of online collaborative tools and platforms available for the public servants, and online training to enable public organisations to quickly adopt this new way of working.

Table 3.4. Ways of working that create an environment to encourage digital transformation

\begin{tabular}{l|l}
\hline What supports digital transformation & What doesn't support digital transformation \\
\hline Agile and user-centred working methodologies & Rigid and product-centred working methodologies \\
\hline Adequate tools and technologies & Blocked software and slow computers \\
\hline Flexible work space (office or non-office) & Traditional office space \\
\hline Flexible working policies & Resistance to remote/flexible working \\
\hline
\end{tabular}




\section{Defining skills for a digitally enabled state}

The OECD Framework for Digital Talent and Skills in the Public Sector consists of three interconnected elements. The previous chapter considered the first of the framework's three pillars and this chapter will focus on the central element of the framework, the blend of skills needed to support digital government maturity (Figure 4.1). This chapter builds on existing work discussed in the Introduction to define and support digital skills at the societal level and efforts to ensure digital divides in society are reduced rather than exacerbated. These have provided the basis to think about the needs of skills within the public sector workforce at the organisation and sectoral level for a high performing civil service and the individual and team levels for leadership and innovation (OECD, 2017 $7_{[13]}$; OECD, 2017[12]; Gerson, 2020[14]).

Figure 4.1. The OECD Framework for Digital Talent and Skills in the Public Sector (focus on Pillar 2)
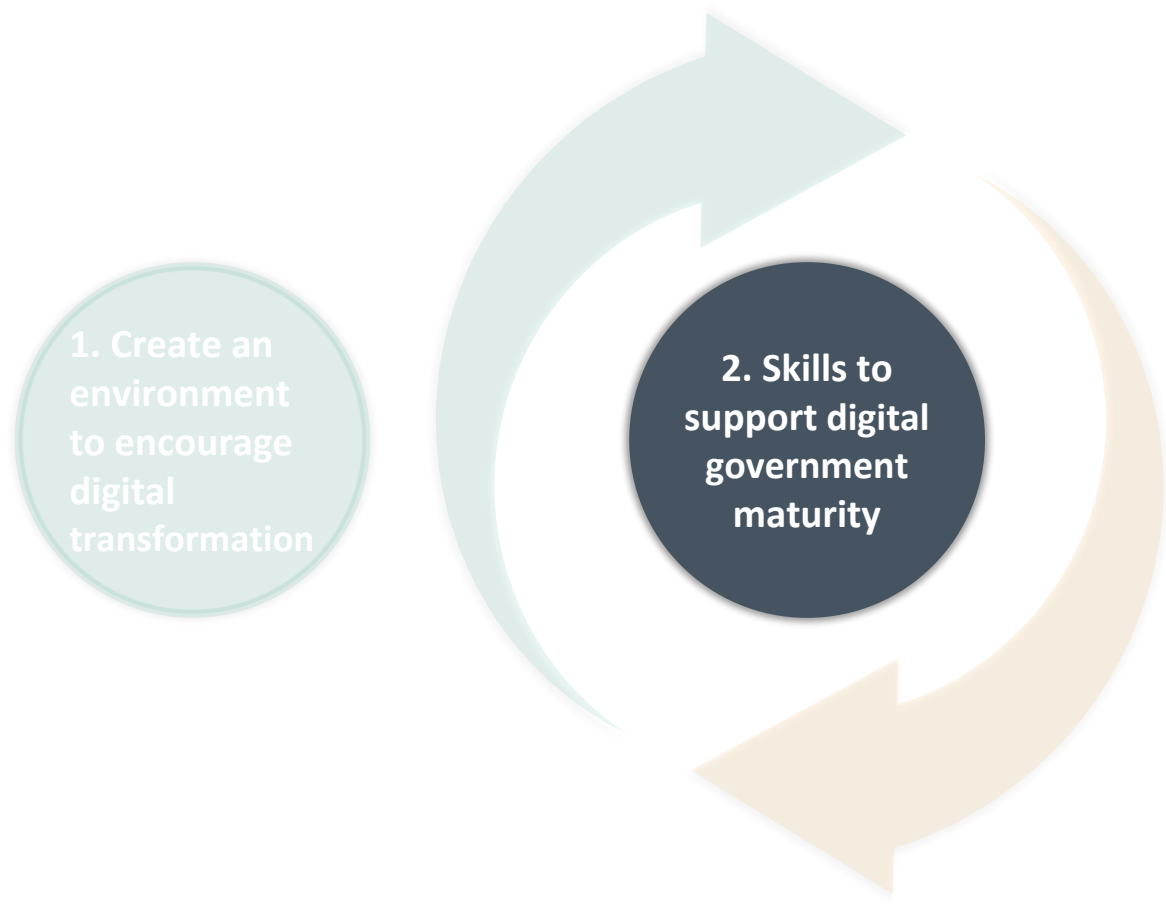

Source: Authors' own design

This chapter discusses five layers of skills required to support digital government maturity (Figure 4.2). The starting point is a baseline for $21^{\text {st }}$ century skills in society. Digital government efforts build on this foundation in four areas: digital government user skills, digital government socio-emotional skills, digital government professional skills and digital government leadership skills. In doing so, this chapter argues 
that while every citizen should have a baseline of $21^{\text {st }}$ century skills, a similar expectation should exist for every public servant to have foundational digital government user skills, no matter their role or position in their organisational structure or whether their work relates to a national or local tier of government. This will establish a core understanding about the potential for, and application of, digital government thinking and practice throughout the public sector, not just among those in digital government teams or operating in designated digital or ICT roles.

\section{Figure 4.2. Skills to support digital government maturity}

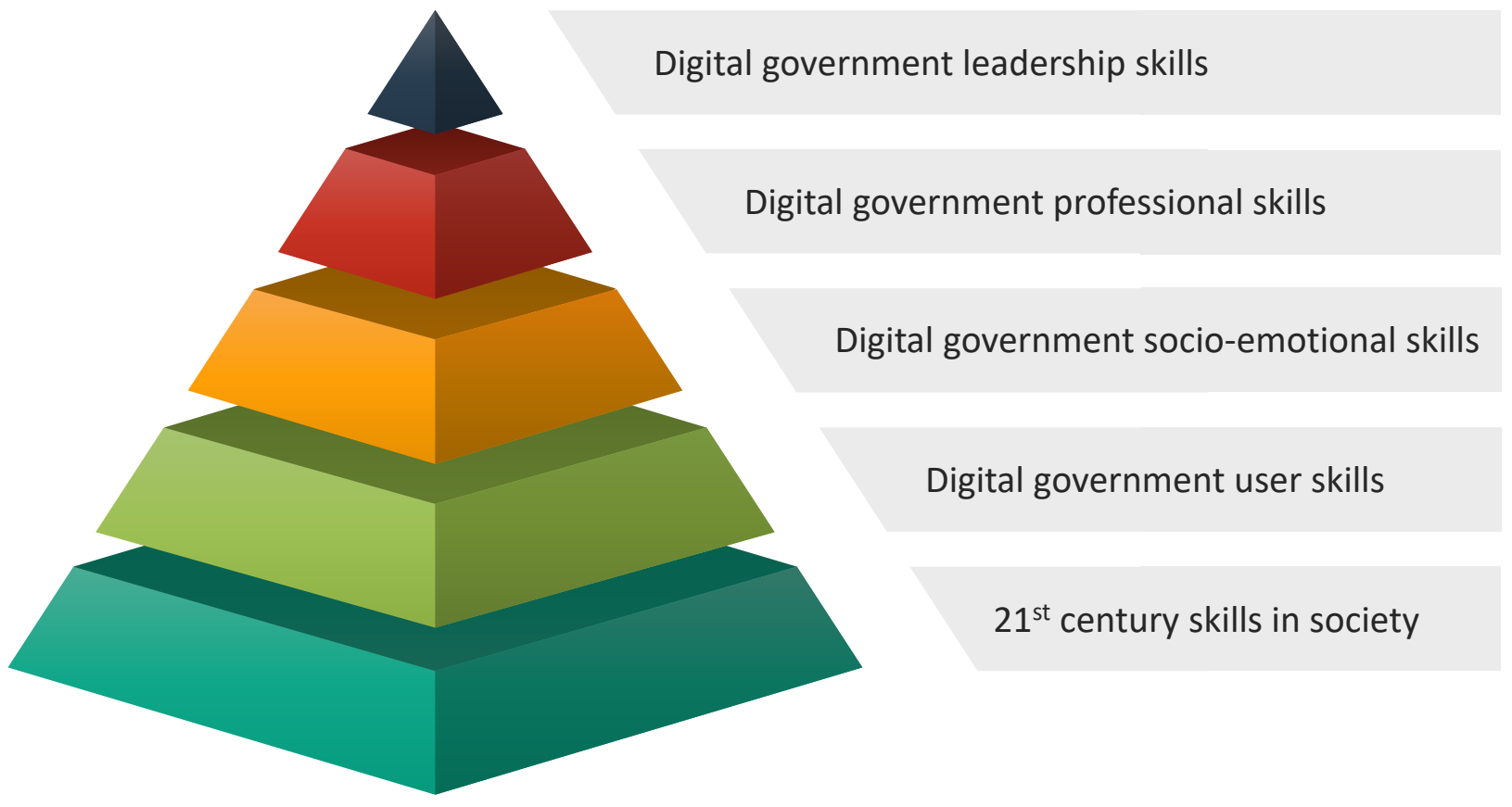

Source: Authors' own design

$21^{\text {st }}$ century skills in society

\section{Society as a whole is capable and equipped with the necessary digital, cognitive and socio-emotional skills to thrive in the digital age. This is core for all to have basic confidence in using digital tools and technologies. All efforts to establish a digitally enabled state rely on this grounding.}

Skills-related policies that successfully equip people with a broad mix of skills will ensure the technological revolution improves the lives of all. It is particularly important to ensure that the promise of digital transformation does not widen existing inequalities or create new ones as some jobs disappear and some skills become outmoded (OECD, 201999]). Therefore, addressing the skills gap in society is critical to avoid exacerbating, or creating, inequality in terms of access to the benefits of digital transformation whether through socio-economic, demographic, generational, geographic, educational or infrastructural challenges.

The ambition must be for two things to be achieved. Firstly, that over time society as a whole becomes more capable and equipped with the necessary breadth of skills to thrive in the digital age. Secondly, that 
alongside a recognition of the need to upskill to a particular benchmark there would be efforts to encourage a continuous, and life-long approach to these skills which ensures society remains equipped on an ongoing basis. As benefits are seen in terms of the digital economy, benefits will also flow into the public sector as its own workforce demonstrates their own foundational digital skills.

At its most basic, the notion of digital user skills indicates an ability to use Internet connected devices. The baseline for what constitutes 'an ability to use' is fluid and it is therefore important to avoid overly simplistic framings and instead reflecting changing habits, purposes and needs. Nevertheless, it is important to find a definition that points towards realistically deriving benefits from digital technologies. One model for understanding and defining digital literacy and digital skills in society is the European Digital Competence Framework 2.0 (DigComp) which looks at five areas of digital competencies (Box 4.1). In Slovenia, the Administration Academy at the Ministry of Public Administration launched a new "Digital literacy training programme for public servants" in 2019. This programme follows the DigComp Framework for Citizens with 21 competences in five areas. The objective of the training programme is to enable civil servants to use information and communication technologies in a creative, safe and critical way. This follows from the 2018 launch of a "Data management" programme which consists of different modules tailored for different focus groups such as managers, analysts and IT experts with varying degrees of knowledge. The objective of the training is to foster data literacy and use of modern technologies for better decision making. Both programmes could be performed either in person or remotely.

\section{Box 4.1. 5 areas of the European Digital Competence Framework explained}

1. Information and data literacy: To articulate information needs, to locate and retrieve digital data, information and content. To judge the relevance of the source and its content. To store, manage, and organise digital data, information and content.

2. Communication and collaboration: To interact, communicate and collaborate through digital technologies while being aware of cultural and generational diversity. To participate in society through public and private digital services and participatory citizenship. To manage one's digital identity and reputation.

3. Digital content creation: To create and edit digital content. To improve and integrate information and content into an existing body of knowledge while understanding how copyright and licences are to be applied. To know how to give understandable instructions for a computer system.

4. Safety: To protect devices, content, personal data and privacy in digital environments. To protect physical and psychological health, and to be aware of digital technologies for social wellbeing and social inclusion. To be aware of the environmental impact of digital technologies and their use.

5. Problem solving: To identify needs and problems, and to resolve conceptual problems and problem situations in digital environments. To use digital tools to innovate processes and products. To keep up-to-date with the digital evolution.

Source: European Commission, (n.d.[54]), The Digital Competence Framework 2.0; Vuorikari et al, (2016[55]), Update Phase 1: The Conceptual Reference Model DigComp 2.0

Earlier models for skills in digital government incorporated these user skills as being requisite for the public sector in terms of productivity in using digital tools for word processing or email. This paper proposes that these skills should instead be explored as part of a baseline competency for society in general. Although different countries are at different levels of digital maturity within society and within the public sector, 
reaching a universal standard for digital literacy within both the country and the public sector itself should be a policy priority distinct from the specific challenges of digital government.

However, 'digital literacy' is one part of what societies need in terms of skills for the $21^{\text {st }}$ century. A 2008 model for ' $21^{\text {st }}$ century skills' developed by The Partnership for $21^{\text {st }}$ Century Skills envisaged a framework that brings together technical information, media and technology skills with cognitive skills for learning and innovation as well as the socio-emotional aspects of life and career (Partnership for 21st Century Skills, $\left.2008_{[56]}\right)$. More recent analysis by van Laar et al $\left(2017_{[57]}\right)$ underscores the importance of developing an understanding of 21 st century skills that blends a more technical understanding of tools with the practicalities of being able to think, work and live. When it comes to the classroom, the OECD Learning Compass 2030 identifies the importance of preparing students to enter the workforce with mastery of cognitive and meta-cognitive, social and emotional, and practical and physical skills that will over time allow for a transformation of the workforce ${ }^{6}$ (OECD, 2019 $\left.[10]\right)$.

The introduction to this paper highlights that the OECD's wider work on skills for the home, in education or at work has identified the core foundations for life in the $21^{\text {st }}$ century to involve digital and data literacy as well as cognitive and socio-emotional skills (OECD, 2019 ${ }_{[10]}$; OECD, 2019 $9_{[9]}$ ). It is therefore important to recognise that a definition of ' $21^{\text {st }}$ century skills' must consider more than the technical aspects of 'digital literacy' in order to flesh out the full competencies needed for society to thrive in the digital age.

\section{Digital government user skills}

\section{Built on competency in society, these are the 5 areas of core skill needed for all public servants to support digital government maturity: Recognising the potential of digital for transformation, Understanding users and their needs, Collaborating openly for iterative delivery, Trustworthy use of data and technology, and Data-driven government}

Achieving a baseline of $21^{\text {st }}$ century skills for every member of society is a critical prerequisite for supporting digital government maturity. Ensuring that citizens (and by extension public servants) are equipped with the necessary skills and competencies to thrive in the $21^{\text {st }}$ century must be a policy priority for countries, and seen as the foundation for efforts to develop the digital government user skills required for realising the benefits of digital transformation.

Digital government user skills should be considered as a core element of the skills for public servants, regardless of their role or tier of government, in order to be effective in supporting a digitally enabled state. This is critical because while the success of broader digital skills policies will determine the basic competency of a public servant in terms of $21^{\text {st }}$ century skills, nobody is expected to join the public sector workforce with a strong understanding of digital government. Therefore, an equipping in core digital government skills will add value, irrespective of a person's personal or professional background.

Government's use of digital technologies, digital practices and data are critical in helping countries to improve people's lives, across the whole of society. Digital government is not about putting what was on paper onto the internet but about re-engineering and re-designing services and processes so that they are

\footnotetext{
6 The Learning Compass 2030 identifies cognitive and meta-cognitive skills as including critical thinking, creative thinking, learning-to-learn and self-regulation; social and emotional skills as including empathy, self-efficacy, responsibility and collaboration; and practical and physical skills as including using new information and communication technology devices (OECD, 2019[10]).
} 
digital in their design. Doing this means a radical shift away from top-down assumptions about solutions according to government needs to instead take a user-driven approach. By empowering teams to engage and collaborate with citizens, businesses and other stakeholders, government can understand and address their needs, supported by technology and data.

To achieve these outcomes, governments need a workforce that can understand and apply these ideas. The skills for supporting digital government maturity are more than ensuring the necessary digital literacy to be able to use tools and technology; they require a multi-faceted understanding of the potential for digital, data and technology to transform the functioning of government and better meet the needs of the public. As one of the greatest challenges to sustainable digital government is achieving ongoing and consistent support between political cycles, it is important to embed a deep understanding of digital government amongst all those involved with policy and service design and delivery, regardless of their role. Making these ideas part of the fabric of government in this way can help to change not only the culture, but the activity of government too.

Establishing the necessary skills for public servants to prove successful in understanding and applying digital government practices necessitates a model that can scale. There are many enablers to help establish this culture but one of the most effective has been the development of service standards that provide a clear and effective definition of quality and that can then guide subsequent delivery such as those discussed in Box 4.2 (OECD, 2020[27]). A further area of individual and team level thinking that informs the exploration and understanding of the foundational skills required by public servants is the OECD's Core Skills for Public Sector Innovation (2017 [12] $_{\text {) }}$ which identifies six categories of skills based on research with civil servants involved in innovation projects, activities and teams from around world, they are: iteration, data literacy, user centricity, curiosity, storytelling and insurgency (see Figure 2.6 in the Introduction)

Drawing together these ideas and building on the deliberations of The Working Party of Senior Digital Government Officials (E-Leaders) Thematic Group on Digital Talent and Skills this paper proposes a foundational set of competencies across five areas that will ground every public servant in digital transformation (Figure 4.3). The content which follows provides an initial starting point for identifying these areas, further work is needed to develop a more detailed rubric for implementation and the complementary tools with which governments can assess their workforce capability.

\section{Figure 4.3. Digital government user skills}

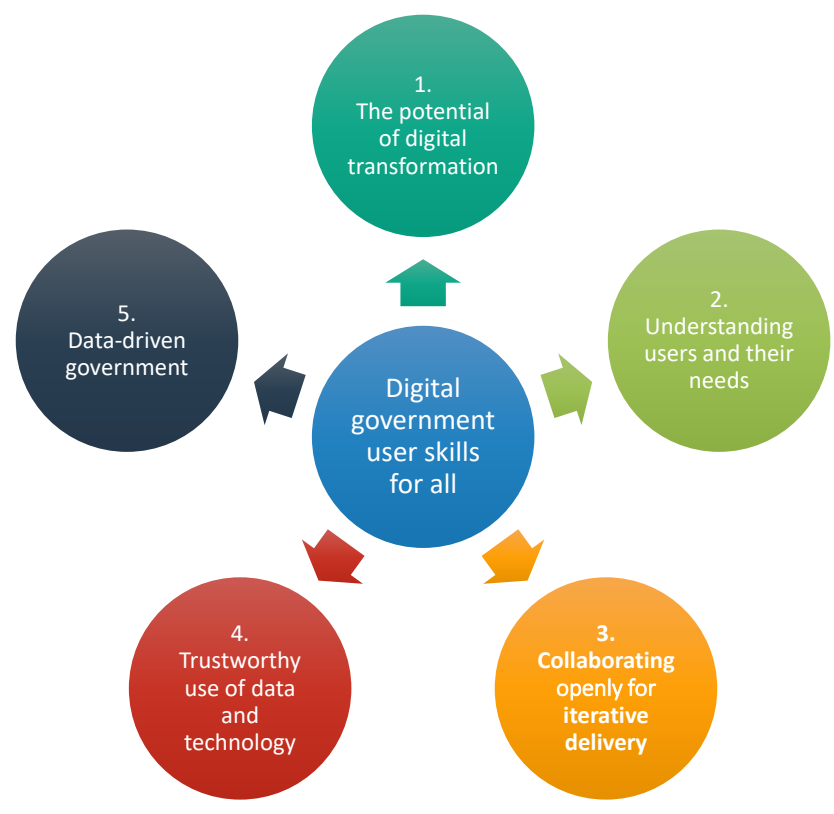

Source: Authors' own design 


\section{Box 4.2. Overarching principles for designing, and delivering, services}

\section{Proposed General Principles for Digital Service Delivery}

Under the auspices of the Working Party of Senior Digital Government Officials (E-Leaders), OECD member countries have been considering what constitutes best practice in this area for several years. At the 2017 meeting in Lisbon, Portugal, the Thematic Group on Digital Service Delivery presented a set of General Principles that both member countries and other governments could follow. These principles emerged from the experiences of member countries in implementing their digital agendas.

1. User driven - Optimize the service around how users can, want, or need to use it, including cultural aspects rather than forcing the users to change their behaviour to accommodate the service.

2. Security and privacy focused - Uphold the principles of user security and privacy to every digital service offered.

3. Open standards - Freely adopted, implemented and extended standards.

4. Agile methods - Build your service using agile, iterative and user-centred methods

5. Government as a platform - Build modular, API enabled data, content, transaction services and business rules for reuse across government and 3rd party service providers

6. Accessibility - Support social inclusion for people with disabilities as well as others, such as older people, people in rural areas, and people in developing countries.

7. Consistent and responsive design - Build the service with responsive design methods using common design patterns within a style guide

8. Participatory process updating - Design a platform to take into account civic participation in the services updates.

9. Performance measurements - Measure performance such as Digital take-up, User satisfaction, Digital Service Completion Rate and Cost per transaction for a better decision-making process.

10. Encourage Use - Promote the use of digital services across a range of channels, including emerging opportunities such as social media.

Source: Proposed General Guidelines for Digital Service Delivery prepared for the 2017 meeting of the Working Party of Senior Digital Government Officials (Unpublished)

\section{Recognising the potential of digital for transformation}

The starting point for digital government user skills is as inspiration for the public sector workforce to recognise the potential of digital for transformation and as a result understand the relevance of the subsequent dimensions. Critical to the success of digital government efforts is a shared vision across the public sector for the opportunity provided by digital technologies and data. Helping to establish a common mindset amongst public servants is crucial in using digital technologies and data to help rethink and redesign government in ways that respond to the needs of the public. This reflects points 5 (Government as a platform) and 7 (Consistent and responsive design) of the Proposed General Principles for Digital Service Delivery (Box 4.2) as well as echoing elements of the curiosity, insurgency and storytelling dimensions of the Core Skills for Public Sector Innovation (OECD, 2017 $\left.{ }_{[12]}\right)$. . This theme also most closely aligns with the Digital by Design, Government as a Platform and Proactiveness dimensions of the Digital Government Policy Framework (DGPF) (Box 2.1). 
As has been discussed, digital government user skills are not solely for those working in areas directly impacted by digital in government, nor are they for new joiners to the civil service. Many public servants have been in the same programme area for a long time and don't know what might be possible as a result of digital transformation. These ideas are valuable for all public servants, regardless of the role they perform, their length of service or position in the organisation hierarchy.

This first element is therefore about establishing a common vision for digital transformation and showcasing how digital and data have been transformative to the effectiveness, efficiency or experience of their colleagues and their stakeholders throughout the public sector whether working on policy development, service delivery or ongoing operations.

As well as benefitting from the inspirational experiences of users and delivery teams in transforming the experience of government, this area of skills includes domain-knowledge of the broader strategic efforts to support digital government as well as more specific practical understanding of the value and utility of enabling resources and tools being developed in the public sector to help accelerate and scale the pace of transformation. It is not necessary for all public servants to have a detailed understanding and knowledge of how to access all the enablers discussed in Box 4.3 but awareness of them is critical for embedding digital government throughout the public sector workforce.

\section{Box 4.3. Enablers to support the digital transformation of services at scale}

Best practice guidance that offers wisdom and insight into practising digital government such as style guides, service manuals and other documentation.

Service standards and assurance processes that establish what 'good looks like' for public servants across government in terms of the design and delivery of services.

Funding or commissioning digital projects to ensure knowledge of business case processes, procurement frameworks or technology standards.

Digital inclusion focused activity being carried out around prioritising digital literacy, connectivity and accessibility to ensure digital government does not worsen any 'digital divides'.

The channel strategy for replacing any patchwork of independent entry points to government with an omni-channel model of service provision to ensure no matter the channel someone chooses, they will always be able to access a consistent, joined-up and high-quality service

Common components and tools that are available to address common user needs such as design patterns, identity, notifications, payments, or web infrastructure.

Data-driven public sector approaches in line with the recommendations of the OECD's framework for becoming a data-driven public sector (OECD, 2019 $\left.{ }_{[58]}\right)$.

How to access capability in terms of talent and skills as described in this working paper to address needs around recruitment, retention, training and working with the private sector.

Source: OECD, (2020[27]), Digital Government in Chile - Improving Public Service Design and Delivery 


\section{What it means for a public servant to recognise the potential for digital transformation}

Able to identify, describe and analyse practical examples of digital transformation

Able to look at the status quo of existing processes and identify opportunities for digital transformation Have a growing network of digital government practitioners to turn to for advice and challenge

When made aware of new activity involving technology they understand, and can challenge, whether it is consistent with wider strategic activity

If responsible for new digital, data or technology activity they oversee and develop it in ways that complement broader strategic activities

When involved with oversight of digital, data and technology activity they are able to ask relevant, informed and challenging questions to go beyond superficial reporting

\section{Understanding users and their needs}

The second area to consider in developing the skills to support digital government maturity is around equipping public servants to identify, and understand, their users and their needs. This reflects points 1 (User driven), 6 (Accessibility) and 7 (Consistent and responsive design) of the Proposed General Principles for Digital Service Delivery (Box 4.2) as well as echoing elements of the storytelling and user centricity dimensions of the Core Skills for Public Sector Innovation (OECD, 2017 $7_{[12])}$. . This theme most closely aligns with the User-Driven dimension of the DGPF (Box 2.1).

In order to achieve transformation it is inadequate to focus on individual interactions in isolation from an understanding of the whole problem. To design services that respond fully to the needs of users it is critical to carry out user research to map and understand the existing landscape of government service provision, the interactions and data flows between institutions and the involvement of the public. This ensures responses reflect a multi-faceted understanding of the problem as it manifests in reality rather than informed by desk-based assumptions. Responding to what has been found in order to better meet needs may then require a fundamental redesign of the service, or more minor tweaks to the way in which government is working in order to improve results.

An important element of this process is to be aware that many user journeys are convoluted and circuitous, often crossing organisational boundaries and moving between different channels and modes of delivery. This reflects the reality of how government services have evolved over time with different policy initiatives leading to a public experience fragmented across various channels and internal processes that are little better. By contrast, an important marker of digital government maturity is joined up, proactive and seamless government and therefore, public servants should develop a knowledge of service design thinking to identify transitions between physical, offline and digital elements and understand how to improve the end to end experience for both the public and their colleagues.

Finally, in order to understand users and their needs, public servants must become champions for ensuring services are available to the whole of society by understanding the importance and strategies for increasing accessibility, connectivity infrastructure, and the $21^{\text {st }}$ century skills of society (OECD, 2020[27]). 


\section{What it means for a public servant to understand users and their needs}

Can champion and explain the value of user research and participate as part of user research exercises

Can identify the users affected by their area of work, and define the user needs their work meets

Can identify where their activity of work interacts with, receives from, or hands off, to another part of government and recognises the importance of an end to end understanding of the user's journey

Recognises the different channels and modalities involved in the provision of a service and is able to map the user's journey, including associated internal activity

Understands the importance of tackling the digital divide and is consequently aware of the priority, roadmap and strategy for accessibility, national connectivity and increasing $21^{\text {st }}$ century skills in society

\section{Collaborating openly for iterative delivery}

The third area of skills to support digital government maturity is preparing public servants to involve the public in designing and delivering policies and services. This reflects points 3 (Open standards), 4 (Agile methods) and 8 (Participatory process updating) of the Proposed General Principles for Digital Service Delivery (Box 4.2) as well as echoing elements of the curiosity, insurgency, iteration and user centricity

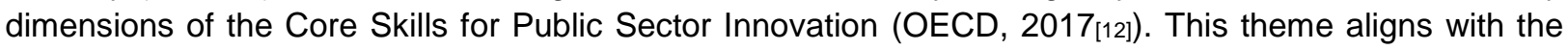
Open by Default dimension of the DGPF (Box 2.1) as well as the Recommendation of the Council on Open Government (OECD, 2017[59]) encouraging greater openness with the public through participatory forms of consultation and teams sharing progress, performance and problems with the public.

This skill builds on the initial understanding of user-centred design developed through 2 . Understanding users and their needs to cement clarity about the role of the public in co-design. Digitally transformed public services need to involve the public early and often to ensure the design process reflects their views, needs and aspirations from the outset and throughout. These exchanges will show public servants the value to users of making incremental, iterative, improvements to policies and services.

A further aspect of open collaboration that all public servants should have a rudimentary appreciation of are the benefits of open-source code and the merits of 'working in the open'. Although the specific technical skills are part of certain Digital government professions, it is important to embed an awareness of suitable applications of open-source code and the availability of common standards, components and patterns as well as the communities that ensures access to these enablers.

Establishing a culture of meeting needs involves changing approaches to delivery. Government needs greater agility in order to embrace uncertainty, respond to changing needs, work across organisational and professional boundaries and operate on the basis of continuously learning and improving. Although the detail of methodologies such as Agile are most relevant to Digital government professionals, all public servants would benefit from developing an appreciation for iterative models of delivery and the merits of starting small to research, prototype, test and learn on an ongoing basis.

Finally, this skill reflects the importance of bringing policy, delivery and operations together throughout the implementation lifecycle to ensure a common vision and coordinated development process. Transformed services rely on diverse, multi-disciplinary teams of designers, developers, subject matter experts, policy officials, lawyers, operational staff, user researchers and content professionals that bring together different perspectives and commit to working across organisational boundaries. A cross-discipline approach involving those from across government helps to better understand the needs of all users. 


\section{What it means for a public servant to collaborate openly for iterative delivery}

Can explain the benefits of 'working in the open' and argue positively for an open by default approach

Can implement participatory approaches with their users to their area of work whether in terms of codesign or other participatory activities

Understands the value of diverse, multi-disciplinary teams and has the expectation of involving policy, delivery and operational colleagues to ensure a fully-rounded perspective on a given topic

Can explain the benefits of an iterative approach to delivery. Understands different phases of delivery (eg Discovery, Alpha, Beta, Live) and is clear about the benefits of researching, prototyping, testing and learning on an ongoing basis

Has an understanding of open source code and the community-based processes that support them

Knows where to find, and appraise, the suitability of common standards, components and patterns

\section{Trustworthy use of data and technology}

The fourth area considers the nature of trust in the context of digital government. This reflects point 2 (Security and privacy focused) of the Proposed General Principles for Digital Service Delivery (Box 4.2). This theme aligns with the public trust element of the Data-Driven Public Sector dimension of the DGPF (Box 2.1).

This dimension is inwardly focused on the behaviour of public servants. The starting point is one that is usually considered as part of an organisation's HR policies in terms of an employee's responsibility in the workplace around information security and data handling or processing as well as digital security in terms of password policies.

In the digital era, there are increasing opportunities for developing personalised services that reflect our Internet browsing habits and can be tailored to our specific circumstances. Designing services that increase convenience while also preserving the trust of users is important and public sector employees are increasingly creators, or custodians of data regardless of their role. The personal responsibility for the way individuals conduct their internal behaviours at work therefore extends to the importance of acting ethically and responsibly in executing their responsibilities. This would include understanding legal requirements in terms of privacy for those whose needs they are addressing as well as considering the ethical dimensions of any use of digital technologies or data. Although the guidelines and codes around the behaviour of public servants likely account for this activity, the challenges of working in the digital era may require additional contextualisation to provide the greatest support to public servants. The Working Party of Senior Digital Government Officials (E-Leaders) Thematic Group on the Data-Driven Public Sector is developing a set of Good Practice Principles for Data Ethics in the Public Sector (GPPs) that aim to help address some of the gaps in this area.

The final dimension for the trustworthy use of data and technology is ensuring the operation of a reliable service. Government services must be available 24 hours a day, 365 days a year. Although maximising uptime and speed of response is the focus of more specialist digital professionals, it is important to establish a basic understanding of the importance of being able to make regular deployments, access to testing environments and addressing contractual arrangements that may prevent users getting the help they need, when they need it. 


\section{What it means for a public servant to be trustworthy in their use of data and technology}

Understands their responsibilities in the workplace around information security and data handling or processing

Is confident in terms of digital security and clear about password policies.

Understands the legal requirements on them as individuals in terms of their handling of data to protect the privacy of citizens

Is comfortable considering the ethical dimensions associated with the use of digital technologies or data, including knowledge of any relevant instruments such as Good Practice Principles

Understands the support and activities associated with maintaining a reliable service

Ensures that contracts with third party suppliers are consistent with the digital government agenda

\section{Data-driven government}

Although data scientists and other analytical roles are an important specialism within the framing of digital government professions, there is a core competency of data literacy that, if distributed across the entire public sector workforce, can underpin those more advanced specialisms and achieve the ambition for a truly data-driven public sector (OECD, 2019[58]). This final area reflects points 3 (Open standards) and 9 (Performance measurement) of the Proposed General Principles for Digital Service Delivery (Box 4.2) as well as echoing elements of the data literacy dimension of the Core Skills for Public Sector Innovation (OECD, 2017 $[12])$. This theme most closely aligns with the Data-Driven Public Sector and Open by Default dimensions of the DGPF (Box 2.1).

Building on some of the discussions about open government in 3. Collaborating openly for iterative delivery and the Recommendation of the Council on Open Government (OECD, 2017 ${ }_{[59]}$ ), this aspect of core digital government competency embeds an appreciation and understanding of the benefits and opportunities for Open Government Data (and open data more generally) in terms of transparency and as a source of public value and stimulant for innovation in the economy.

A basic understanding and appreciation of any standards relating to data architecture should also be shared with public servants. Whilst only digital professionals will be involved in the design of services that capture data, a recognition of the government data value cycle and the existence of any standards as applicable can still help to embed a data culture that commits to improving the quality and availability of data within government and for re-use.

Equally valuable is an understanding of national data infrastructure in terms of sourcing datasets or unlocking the potential value of interoperability platforms and ensuring that as far as possible, duplication of effort or data capture and storage is minimised. Although the specifics of ensuring that will again be the domain of digital professionals, the prize of achieving a whole of government commitment to data interoperability, sharing and re-use necessitates a base level understanding amongst everyone.

All public servants should be encouraged to develop their own ability to understand the potential for applying data in their daily work to generate public value in terms of design, delivery or evaluation (OECD, $2019_{[58]}$. Equipping all public servants with the abilities to source data, carry out analysis, mitigate bias, and define actionable metrics for measuring success, outcomes or impact could contribute to significantly increased public value. 


\section{What it means for a public servant to know about data-driven government}

Aware of the individuals or organisations that are responsible for the data agenda

Understands the priority, roadmap and strategy for taking the steps to establish a data-driven public sector

Familiar with the governance arrangements for access to and sharing of data

Builds on their knowledge over the trustworthy use of data to be confident in their legal and ethical obligations for the treatment of data

Recognises opportunities for how interoperability, the Once Only Principle and access to transactional data can support the better design of services

Adopts an empirical approach to the use of data for generating public value in terms of Anticipating and planning, Delivery, and Evaluation and Monitoring

Understands the value of Open Government Data to government, and the wider ecosystem.

\section{Digital government socio-emotional skills}

\section{Achieving digital government maturity involves championing and ensuring a blend of domain specific socio-emotional skills and their associated behaviours. Striking a balance between vision, analysis, diplomacy, agility and protection is essential to the design and delivery of trustworthy and proactive services that put users at their heart.}

As discussed in Chapter 2, the environment for encouraging digital government values multi-disciplinary teams to bring different professional perspectives as well as ensuring government reflects the diversity of the society it serves in terms of background experience and socio-emotional skills. This reflects the importance within wider society of developing policies that encourage the mix of cognitive, digital and socio-emotional skills, and the associated behaviours, needed to thrive in the $21^{\text {st }}$ century (OECD, $2019_{[9]}$ ).

The skills for digital government discussed above contribute to digital government maturity but successfully embedding a shift in the culture of government requires teams to be established that reflect a diversity of socio-emotional skills and their associated behaviours. Although there are existing models for identifying and understanding the role of socio-emotional skills in the classroom and on into working life (Chernyshenko, Kankaraš and Drasgow, 2018[60]), the OECD's work on Digital Government and Data has highlighted five socio-emotional skills and their associated behaviours which contribute to digital government maturity (Figure 4.4). Unlike digital government user skills there should be no expectation for every public servant to become an expert in all these socio-emotional skills with a focus instead on ensuring a balance of these perspectives in the teams that work on designing and delivering government services. 
Figure 4.4. Digital government socio-emotional skills and associated behaviours needed by teams

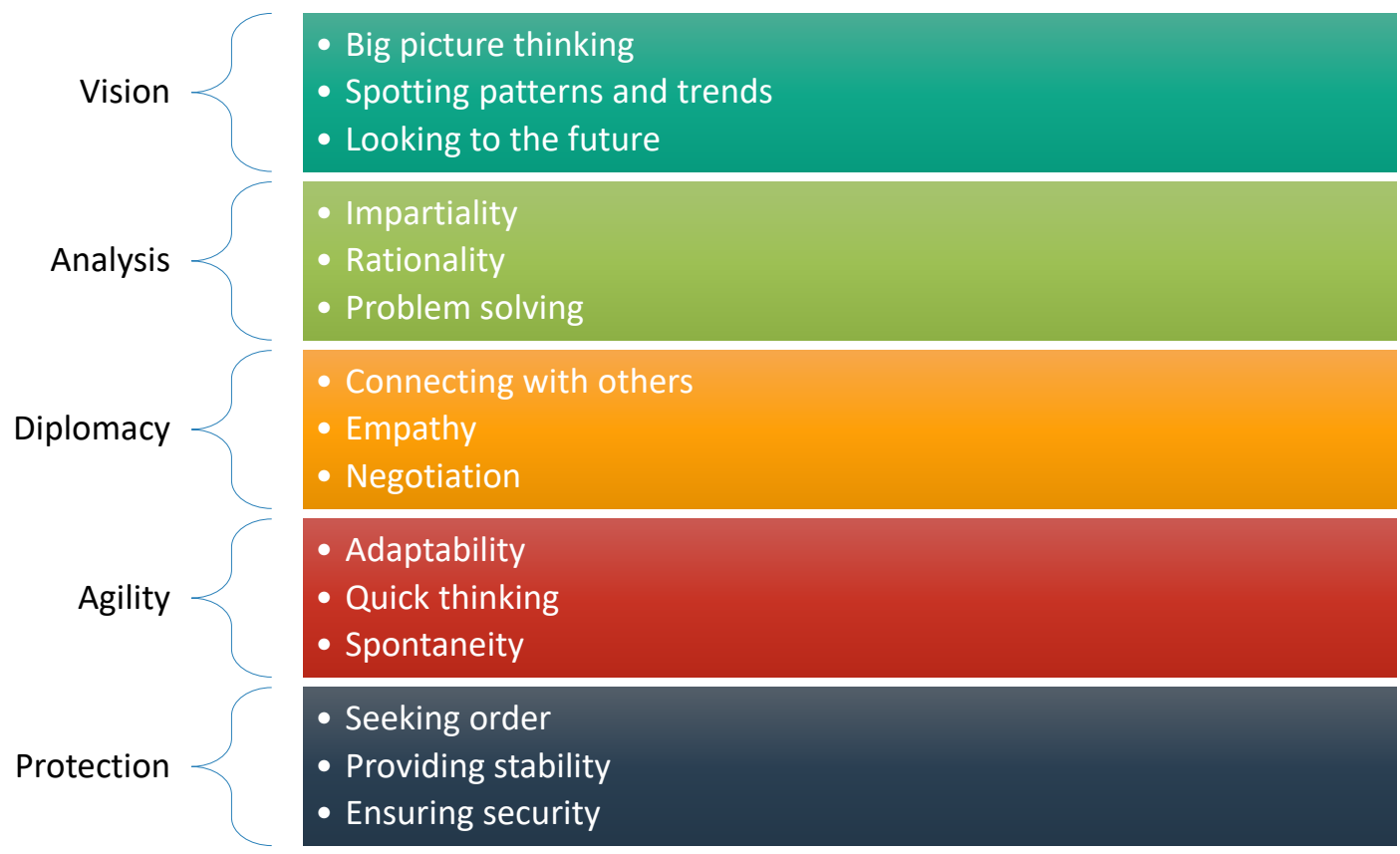

Source: Authors' own design

\section{Vision}

It is important to have visionary characters around digital government teams that are rethinking and redesigning the citizen-government experience. However, it is essential to ensure that those with these skills are paired with individuals whose skills will help to turn imagined vision into deliverable reality. These skills can help to inspire change and build momentum for the digital government agenda. The first way in which this skill manifests is in big picture thinking which reflects a capacity to see the full scope of a system and understand how different moving parts fit together. The second way this skillset adds value is in spotting patterns and trends, whether through being able to interpret the increased quantity of data that is often available following digital transformation, or in understanding the potential for an emerging technology or new way of working. The final area is looking to the future and being able to imagine a sufficiently large idea and communicate it so that others are able to define the role that they can play in achieving it.

\section{Analysis}

Analysis speaks to an individual's capacity to collect information, weigh evidence and respond to the needs that have been found. Those with these skills provide an important complement to the ideas of the visionary members of a team by providing a solid basis for testing assumptions. The first important element of analysis is impartiality, the quality of being able to put to one side the inclination to reach a personal decision and instead focus on understanding the viewpoints of all. Although bias can never be avoided, the conscious effort to acknowledge all experiences with equal consideration is valuable in developing an understanding of the needs of users. The second is rationality, the quality of being able to weigh up and reflect on evidence before forming a clear reason to justify a particular decision. Evidence-based decisionmaking is an important skill within the public sector and one that can increasingly benefit from increased access to higher quality data. The third analytical skill is that of problem solving, the quality of being able to identify and define problems, proposing alternative solutions and weighing them up is an important element of adopting an iterative and experimental approach to public services. 


\section{Diplomacy}

Diplomacy reflects an individual's ability to build relationships, understand those around them and create consensus. The first element is connecting with others to actively seek out opposing points of view and create networks across organisational and team boundaries thereby reducing duplication and facilitating the cross-government resolution of whole problems. The second aspect is empathy to understand user needs, express the emotional intelligence to build rapport and for digital government professionals particularly to acknowledge different levels of proficiency in terms of digital government user skills. The third, negotiation, builds on this empathy to smooth differences between different organisations and professional viewpoints. This is particularly important in the context of multi-disciplinary and crossgovernment activity as opposing points of view are inevitable and makes building consensus and arriving at an agreed outcome critical to success.

\section{Agility}

Agility reflects the importance of continuing to ask questions and react to a fast-changing environment whether in terms of what's being learnt from data and research, or in the pace of technological change. The first element is adaptability and the capacity of an individual to change their actions or approach in response to new information. Change is a constant and the nature of digital government activity might mean reacting to a new situation and smoothly forming a new team at very short notice without jeopardising personal relationships or delivery timelines. The second aspect is quick thinking, a quality that allows the provision of quick and accurate responses in the face of an unexpected situation. The third is spontaneity, an aspect of creativity that allows people to help find solutions to challenges whether newly identified or well established.

\section{Protection}

Protection reflects the importance of addressing the challenges discussed under the Trustworthy use of data and technology digital government user skill around providing trusted, reliable and secure digital government efforts. The first way this adds value is in seeking order, that is the ability to remain calm in the midst of complicated or challenging situations and helping to ensure that a clear way forward is established. The second element is providing stability which is essential in the context of the pressures associated with delivering high profile and large-scale services to the public which benefit from the ability to maintain calm and clarity. The third way this skill manifests is in ensuring security and paying attention to the details of information or digital security to ensure that reliability and trust is preserved.

\section{Digital government professional skills}

\section{The digital transformation has disrupted existing professions and created new ones. Digital government maturity is supported by building multi-disciplinary teams that draw from, invest in and acknowledge both digital and non-digital professions.}

Everybody in society needs to have a baseline of $21^{\text {st }}$ century skills, all public servants need a core understanding of digital government, and there needs to be a blend of socio-emotional skills amongst those working on digital government but the fourth layer of the skills to support digital government maturity narrows the focus to the role of digital and data in the specific professions of the public sector. Multidisciplinary teams need to draw from and acknowledge a variety of professions (Figure 4.5). 
Figure 4.5. Professions involved in a multi-disciplinary team

\section{Digital professionals}

- user-centred design

- product and delivery

- service ownership

- data

- technologists

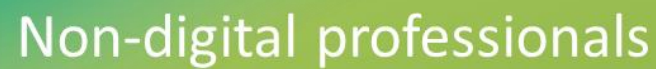

- law, policy and subject matter

- strategy and governance

- commissioning and procurement

- human resources

- operations and customer service

- psychologists and sociologists

Source: Authors' own design

Digital professionals cover the disciplines of user-centred design, product, delivery, service ownership and data as well as digital era technology roles. Each digital government specialism requires its own job family with associated skills profiles and career progression from an initial introduction to the discipline through to leadership roles. The challenges facing governments in terms of recruitment and retention for these disciplines is discussed in more detail in Chapter 4. Honing and developing the skills of each of these disciplines requires targeted training and a set of skills that are already well defined.

- User-centred design professionals include the specialisms of service design, interaction design, content design, and user research. At the most senior level they include Heads of Design and Research who provide strategic direction and vision as well as professional leadership for these different disciplines. Their responsibility is to embed a user-centred culture in the public sector and establish the importance of these disciplines and their allied roles. Proficiency in user-centred design means understanding the needs of users and sharing that insight with the team to ensure a collective recognition of the problems to address in transforming government services.

- Service professionals are senior roles with responsibility for the user experience across product, service and organisational boundaries. Their activities are measured against user-driven and citizen-centric metrics and government outcomes and typically work closely with the leaders of their organisation, and in proximity to ministers. This role ensures accountability for the end-to-end user experience and combats the proliferation of disparate digital services and duplicated spending.

- $\quad$ Product professional roles work at the intersection between different disciplines in a team to ensure a balance between the understood needs of users, the technical feasibility of solutions and the broader organisational context. At the most senior level is the Head of Product taking a perspective across multiple products and services.

- Delivery professionals contribute to successful digital government teams by supporting delivery through activities that involve others in the organisation like financial management and recruitment into the team. They are focused on the health of teams through encouraging and protecting them from external distraction. They will often use Agile or Lean practices to help the team keep a rhythm 
of delivery and learning that ensures value. At the most senior level is a Head of Delivery who is often responsible for supporting all disciplines in an organisation to improve their delivery culture.

- Data professional roles include the Chief Data Officer at the most senior level providing strategic direction and vision for an effective data governance model and ensuring the use of data builds, rather than diminishes trust $\left(\mathrm{OECD}, 2019_{[58]}\right)$. Their responsibility for embedding a data-driven public sector includes acknowledging the importance of roles including data analyst and data scientist. The operational activities of data professionals include managing the data collected through the activity of government, monitoring the performance of public services and drawing insights to inform ongoing delivery or new policy development.

- Technology professionals contribute several disciplines to effective multi-disciplinary teams. At the most senior is the Chief Technology Officer who has responsibility for consistent architectural decisions and the direction of technology practice in an organisation. At an operational level, there are different roles that may be needed including Technical Architects and Developers covering frontend, backend and operations. Technical Architects work with teams to ensure the resilience, scalability and security of the work that teams are doing and helping to make decisions that are consistent with the wider organisation. Developers are needed to build accessible software but to also support the wider activities of the team through advising on technical feasibility, maintaining and documenting code, running production services and solving technical problems.

As discussed earlier in Chapter 2 (under Organisational structure), there is a need for the environment to evolve in order to reflect the full range of professional disciplines that digital era government requires. Several countries are now revising the way in which their job families are defined in order to accommodate a more developed understanding of twenty first century digital, data and technology roles. Although this reflects the need to revise technology roles and ensure recognition of digital professions, it is crucial to recognise the impact of the digital transformation on non-digital specialisms.

The central idea of multi-disciplinary approaches is that all actors are brought together. This means also including traditional disciplines alongside digital specialists. These include policy, legal and subject matter experts, commissioning and procurement, human resources, operations and customer services, and sociologists and psychologists. It is not only explicitly digital professionals that must now operate in the context of a digitally-enabled state, indeed these roles have a significant influence on the operating environment in which digital government takes place. For example, it is critical to establish a mature understanding of digital government amongst human resource professionals to ensure that they are able to design and manage employment and workforce policies that reflect the transformative potential of the agile, user-led, multi-disciplinary and cross-government mindset required to fulfil the ambitions of digital government. Similarly, procurement and commissioning colleagues supporting digital government teams need to be comfortable with ideas around agile and iterative delivery as it implies a very different approach to traditional purchasing. This demonstrates the importance of considering the extent to which any given role of profession requires tailored and discrete capacity building in terms of digital government in addition to the foundational digital government skills discussed earlier. That baseline capacity for all public servants is critical but so too are developing tailored digital government skills for these traditionally non-digital professions.

\title{
Digital government leadership skills
}

\author{
The leadership to establish a digitally enabled state draws on wider \\ investment in the general quality of leadership. However, achieving \\ digital government maturity requires leaders to visibly model digital
}




\section{government user skills and actively shape an environment that encourages digital transformation}

The final element of the skills to support digital government maturity concerns the role of leaders and the particular implications for their skills. Chapter 2 discussed the important contribution of leadership for creating an environment in an organisation to facilitate and encourage greater digital government maturity. One of the important outcomes of that is an empowering organisation that creates space for all members of a team to demonstrate leadership where appropriate. Nevertheless, it remains critical when thinking about the overall skills for digital government to focus on those needed by individuals holding positions of public sector leadership.

The starting point for considering digital government leadership skills are cross-cutting indicators of good leadership in general rather than a focus on a specific domain. At the organisation and sectoral level, the OECD report on Skills for a High Performing Civil Service, identified that some countries treat leadership skills with a distinct focus, while in others leadership forms part of a common, centralised competency framework used by all civil servants (OECD, 2017[13]). At the individual and team level, the OECD framework for Leadership for a High Performing Civil Service considers ability, motivation and opportunity with four competencies focusing on values-based leadership, open inclusion, organisational stewardship, and networked collaboration (Gerson, 2020[14] $)$. Klus and Müller (2020[61]) propose that leadership skills in the digital age need to reflect communication, knowledge and understanding of domain specific language, organisational skills, subject-specific knowledge, digital literacy and self-reflection. They argue that the nature of such leadership is not technical but reflects softer skills with executives being empathetic, openminded, flexible, motivated, and stress tolerant.

These overarching understandings of leadership can be applied generally to leadership in all contexts, including digital government. Nevertheless, in order to deliver on the potential of digital government for transforming outcomes for the public there is a need for public sector leaders to acquire and develop domain specific skills. These skills fall into two categories that reflect areas previously discussed in this paper.

The first category concerns the responsibility of a leader to model and demonstrate the five digital government user skills identified earlier in this chapter. There is nothing particularly unique about these skills in the context of leadership, rather, the profile of a leader is to understand and encourage each of the elements even if they are not experts. The skills of recognising the potential of digital for transformation; understanding users and their needs; collaborating openly for iterative delivery; trustworthy use of data and technology; and data-driven government must not be treated as optional but as an important baseline for all public servants, including and especially public sector leaders.

The second category reflects the embedding of digital government practice in the mindset of public sector leaders as they look to create the right environment and build a digital workforce (as discussed in Chapters 2 and 4). In applying general good practices for leadership and championing digital government they will actively shape the environment, change the structure of an organisation, and improve its effectiveness in ways that are matched to the necessary steps for empowering and creating space for their workforce to do their jobs.

Given the importance of equipping public sector leaders with digital government skills, several OECD countries are investing in targeted programmes. In Australia, the Leading Digital Transformation course supports senior executives to recognise the challenges and opportunities of leading in the digital age to meet the expectations of citizens, business and other users of government services. In Estonia, the Newton Program is designed with modules that develop both participants' leadership and innovation and technological skills to prepare the next generation of senior leaders. While in the United Kingdom, to complement a capability framework for the roles commonly seen amongst senior Digital, Data and 
Technology leadership teams, the GDS Academy runs a course for leaders with a non-digital background to help them understand more about delivering digital services in government.

A further influence on the quality and nature of public sector leadership is the training provided in universities that commonly attract those with the potential to become future government leaders. Recognising this, an international collective of digital government experts has come together to focus on 'Teaching Public Service in the Digital Age'. In doing so they have developed eight Digital Era Competencies that set out what a digital era public service leader should know (Teaching Public Service in the Digital Age, 2020[62]). The Competencies offer a summary that lends itself well to a targeted syllabus for leaders. Although these eight competencies were developed independently of the thematic group and frameworks of the OECD, they align with the most important elements for leaders to consider in terms of their own skills (as compared in Table 4.1).

\section{Table 4.1. Competencies for digital government leadership}

\begin{tabular}{|c|c|}
\hline $\begin{array}{l}\text { Teaching Public Service in the Digital Age Digital Era } \\
\text { Competencies }\end{array}$ & $\begin{array}{l}\text { Alignment with the OECD Framework for Digital Talent } \\
\text { and Skills }\end{array}$ \\
\hline $\begin{array}{l}\text { 1. Values the experience of service users, and can collaborate } \\
\text { with specialists to understand user needs, then design, test, } \\
\text { and adopt effective solutions. }\end{array}$ & $\begin{array}{l}\text { Digital government user skill 2: Understanding users and their } \\
\text { needs }\end{array}$ \\
\hline $\begin{array}{l}\text { 2. Can anticipate and mitigate the privacy, security and ethical } \\
\text { risks that are inherent to governing in a digital era. }\end{array}$ & $\begin{array}{l}\text { Digital government user skill 4: Trustworthy use of data and } \\
\text { technology }\end{array}$ \\
\hline $\begin{array}{l}\text { 3. Understands the need to blend traditional public service } \\
\text { skills with modern, digital skills, and can effectively work within } \\
\text { and lead multidisciplinary teams. }\end{array}$ & Building an environment to encourage digital transformation \\
\hline $\begin{array}{l}\text { 4. Understands the importance of iteration and rapid feedback } \\
\text { loops, and can create a working environment that can } \\
\text { continuously learn and improve outcomes. }\end{array}$ & Building an environment to encourage digital transformation \\
\hline $\begin{array}{l}\text { 5. Can identify the opportunities to improve government } \\
\text { operations, service delivery or policy making, and can } \\
\text { overcome structural and institutional obstacles to change }\end{array}$ & Building an environment to encourage digital transformation \\
\hline $\begin{array}{l}\text { 6. Can use a range of techniques and tools to make } \\
\text { government more open, collaborative and accountable. }\end{array}$ & $\begin{array}{l}\text { Digital government user skill } 3 \text { : Collaborating openly for } \\
\text { iterative delivery }\end{array}$ \\
\hline $\begin{array}{l}\text { 7. Understands how to use data to inform decisions, design } \\
\text { and run services, and create public value inside and outside } \\
\text { government. }\end{array}$ & Digital government user skill 5: Data-driven government \\
\hline $\begin{array}{l}\text { 8. Understands the current and evolving affordances of digital } \\
\text { technologies and can assess how they can be used to improve } \\
\text { public outcomes. }\end{array}$ & $\begin{array}{l}\text { Digital government user skill 1: Recognising the potential of } \\
\text { digital for transformation }\end{array}$ \\
\hline
\end{tabular}

Source: Teaching Public Service in the Digital Age (2020[62]), The Competencies 


\section{Path to a digital workforce}

After developing an environment that welcomes a digital workforce (Chapter 2) and equipping them with the necessary skills (Chapter 3 ), the next step is to retain the current talents and bring in new people with the right profile (Figure 5.1). This chapter focuses on the practical activities that go hand in hand with recruiting, developing and retaining a workforce that can transform and reinforce the digital maturity of a given organisation.

Figure 5.1. The OECD Framework for Digital Talent and Skills in the Public Sector (focus on Pillar 3)
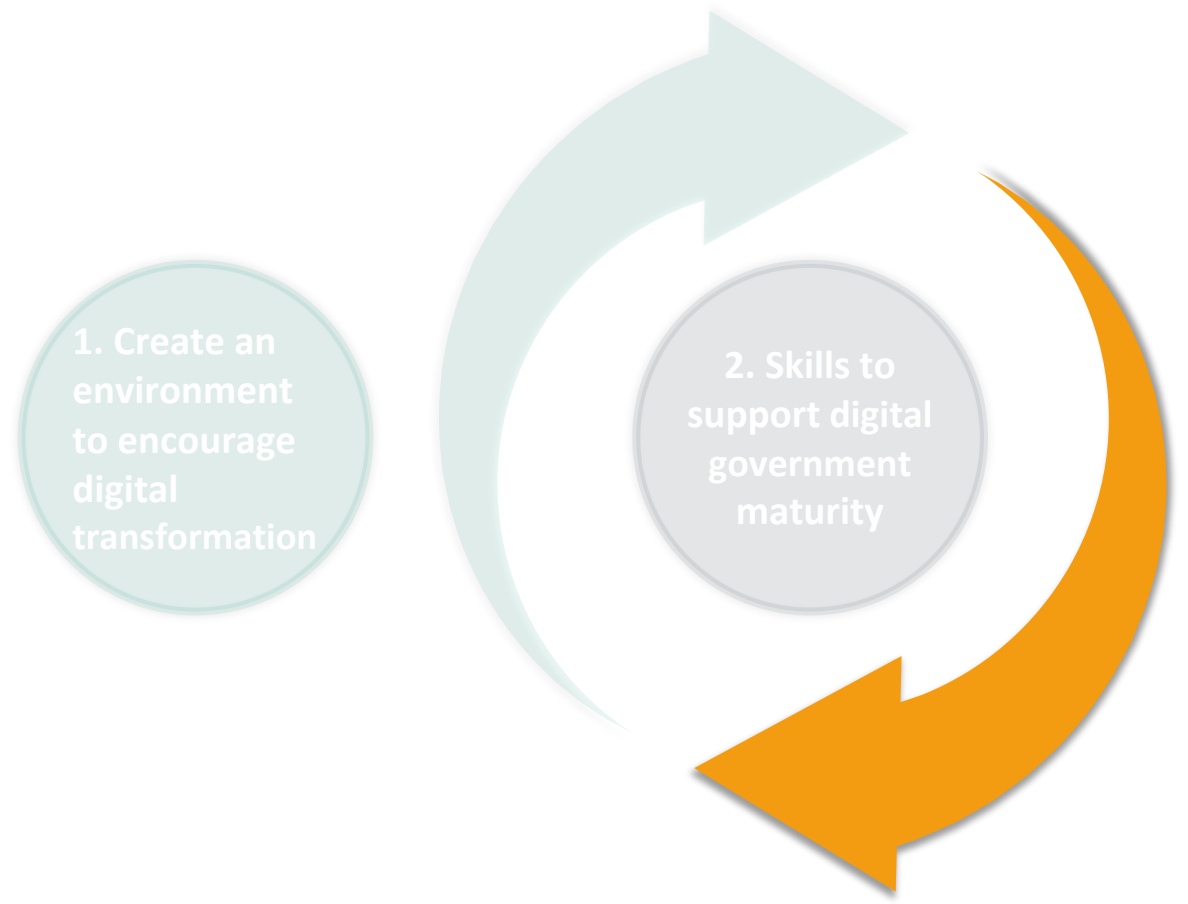

3. Establish

and maintain

a digital

workforce

Source: Authors' own design

Efforts to develop a digital workforce will influence the wider organisation. The activities discussed under this chapter feed into and inform the ongoing environment discussed in Chapter 2 as building a skilled workforce is useless if they don't sit in an environment that is ready for them. As Figure 5.1 illustrates, the arrows indicate that Pillar 1 and 3 are interconnected in a circular motion. This means that limited progress will be possible unless the right conditions exist to start encouraging these activities and that progress will be superficial if these activities do not include a feedback loop to iterate the contextual considerations discussed under Chapter 2.

The OECD's Skills for a High Performing Civil Service report indicates that achieving a highly skilled civil service requires the public sector to attract and select talents, develop skills through training and learning cultures, and make put learned skills to use (OECD, 2017 $[13])$. This paper applies this model to the context 
of digital government, surfacing relevant country practices that demonstrate the direct application of this thinking.

This chapter will first address the attraction of talents and skills. Although the mindset of existing public servants can introduce challenges in terms of the skills profiles discussed in Chapter 3 the retirement of public servants is an opportunity to bring in new hires whose prior knowledge and can complement to efforts to develop in-house expertise. To do this, the public sector needs to promote itself as an attractive employer and invest in recruitment processes that empower candidates to express their competencies. Once talents are hired, organisations need to ensure that their workforce stays motivated. Designing clear career paths and offering a transparent reward system not only maintain a satisfied workforce and expand skills, but also improve well-being at work and decrease turn over in organisations.

Beside this, it is important for the public sector (whether national or local) to have the right skills for building and retaining knowledge in-house, so that organisations can reduce their dependency on third parties. Development of skills can be done formally as well as informally in a flexible and open manner by incentivising the workforce to initiate informal ways of learning and create a peer-to-peer dynamic. Given that the skills of a workforce are the foundation of a successful organisation, ensuring training, skills transfer, communities of practice and high motivation are crucial to enable a digital transformation and keep enthusiasm high.

After the workforce is equipped with digital skills, the right allocation of those talents to tasks is the next challenge. To enable this, organisations need to allow job mobility for employees to work in areas of their interest while building multi-disciplinary teams, to include mentoring programmes and regular feedback from managers to ensure employees' skills and interests match with organisations' strategy.

The COVID-19 pandemic prompted the rapid adoption of new ways of working and the reforms to the working environment and required organisations to rethink how work is carried out, revisit the role of offices and reinvent their approach to work. Since this has reset major work trends, organisations and leaders need to reimagine a work life that is flexible and agile and provide the necessary support to their employees to feel safe and stay productive in a hybrid but primarily digital-first state that can be resilient to current challenges and any future crises.

By improving engagement through more flexible and individualised human resources management, public organisations can create the conditions to attract and retain the right kind of talent to build the public workforce needed in the future while being inclusive and diverse.

\section{Attracting talents}

\section{Attracting the talent for a digital workforce involves establishing recruitment teams to promote the public sector as an attractive employer, investing in recruitment processes and ensuring that recruitment selection stays fair and merit-based.}

Recruitment refers to the process of finding new members to join and work for an entity. However, finding the right candidate for the right role in the public sector becomes difficult as studies show that the public sector labour pool is shrinking over time (Chetkovich, 2003[63]; Lewis and Frank, 2002[64]). The reasons for this are suggested to include the value of job security decreasing over the years while in some countries public servants are negatively portrayed as bureaucratic and "lazy" with slow and unresponsive human resource teams (Collins, 2008[65]; Lewis and Frank, 2002[64]). The OECD's Public Employment and Management Working Party has been considering how to mitigate the damage of these stereotypes on 
the image of a public sector career, with forthcoming work exploring how the public sector can rebrand itself as an attractive employer and create outreach strategies by clearly communicating both the work and the worth of working in the public sector, such as solving problems, delivering outcomes for citizens, and offering opportunities to work on innovative projects.

Recruitment strategies are as important to the public sector as they are to the private sector. They ensure transparency and fairness in the hiring process, which assist hiring managers in selecting the right candidate for the job on the basis of merit. Since research found that the wording used in job advertisements can subtly attract men or women to a profession or industry, organisations need to be aware and avoid gender-stereotyped words, such as 'competitive' and 'dominant' which are masculinestereotyped words and appear less attractive to women (Gaucher, D.; Friesen, J; Kay, A. C., 2011[66]). Therefore, using gender-free language to establish a clear description of the role and communicate the wider offer of an organisation to its employees is vital to try and ensure inclusiveness in hiring practices.

\section{Recruitment teams}

Having a "centralised" recruiting system, where a specialised team is responsible for recruiting qualified personnel across the entire public sector will create a coherent approach to promoting the career of a public servant and attracting candidates through social media, job fairs and school visits. This approach allows organisations to take advantage of recruiters' specialised knowledge and provide an attractive environment for candidates. For example, the Digital, Data and Technology (DDaT) Cross-Government Recruitment Service in the United Kingdom helps to attract, develop and retain the people and skills needed for government transformation. The team offers support in recruitment for all grades including Senior Civil Service roles and helps with:

- designing recruitment strategies - advising on the best routes to market

- branding advice - producing the best job ads and descriptions to attract the right candidates

- access to other government department's reserve lists

- identifying diverse panel members

- jointly running cross-government recruitment campaigns.

Recruitment teams can also help to encourage applications from under-represented groups. For example, in Canada, the Treasury Board of Canada Secretariat and the Office of the Chief Information Officer for Canada Community Management Office has been responsible for a multi-year government-wide commitment to help advance recruitment of Women in Technology through specific recruitment campaigns and placing additional emphasis on hiring women into recruitment campaigns for careers in information management and information technology more widely. These examples show that dedicated teams in charge of promoting the public sector as an employer can help to minimise time and effort in searching, interviewing and hiring. They contribute to building an attractive image for the public sector by effectively communicating the meaningful work they do while targeting potential prospects.

A further way that teams can contribute to recruitment is through partnerships between government and universities encouraging graduates to work for the public sector. In the United States of America, the Volcker Alliance's Government-2-University initiative is an innovative approach to building regional networks of governments and universities (The Volcker Alliance, 2020[67]). Prestigious "fellowship" programmes like this enable governments to work closely with universities and communicate the needs of the public sector.

\section{Recruitment process}

Recruitment processes in the public sector often incorporate additional steps to determine the suitability of applicants for digital positions. In Slovenia, recruitment activity tests against the competency model 
developed in the Public Employees Act. In the United Kingdom, recruitment for roles covered by the Digital, Data and Technology Profession Capability Framework (Box 5.1) may include a technical assessment at the point of interview. A similar test of digital skills forms part of recruitment for Colombia, Ireland and Uruguay. In Singapore, applicants for technical ICT positions in the public sector are assessed against the GovTech Technical Competency Framework through coding tests, pair programming and scenario-based interviews. 'Practice Leads', typically the head of the entire job family, validate senior appointments to support hiring managers without domain expertise in certain areas. Designing recruitment processes that go beyond the hiring manager and HR can help to ensure accountability and greater clarity in determining the skills of an applicant.

\section{Box 5.1. The Digital, Data and Technology Profession Capability Framework}

Designed in 2017 to help improve consistency across Digital, Data and Technology (DDaT) job roles in government, the Framework covers the job families of the DDaT profession (Data, IT operations, Product and delivery, Quality assurance testing, Technical and User-centred design) and provides details of the skills needed to work at each role level. It sets out a common language to describe roles, define associated skills and map career paths.

The DDaT Framework has helped to create and grow cross-government communities of DDaT professionals, identify skill gaps and develop the training offered through the GDS Academy. The Framework can be used to:

- learn about what different roles do in government

- understand what skills are needed by professionals in particular jobs

- identify skills that need development to help career progression

- assess skills in preparation for performance reviews

- create effective job adverts

- carry out Human Resources and workforce planning

Source: Government Digital Service $\left(2019_{[68]}\right)$. Making the Digital, Data and Technology Capability Framework more user friendly

Some sectors have started to use less traditional ways of recruiting and are turning to "game science" or "gamification" to enable candidates to demonstrate skills and solve work situations. The concept is to create an environment where people compete or collaborate to reach an objective, and through the process reveal personalities, strengths and weaknesses, values and behaviours. This can help make recruitment more inclusive by placing candidates in real situations where they can demonstrate their capability and offset some of the biases that research has shown mean female candidates face tougher evaluation of their credentials (Moss-Racusin et al., 2012[69]). The United States of America Army has used games to facilitate their recruitment process since the release of "America's Army" in 2002. In 2008, the "virtual Army experience" was developed and taken on tour around the country and brought the recruiting game to shopping centres, fairs and other locations (Bock, 2012[70]). Gamifying recruitment can not only give candidates a sneak peak of the tasks and situations they may expect to face, but also enable the employer to witness candidates' reactions.

To enhance public sector attractiveness, hiring managers need to embody public sector values of integrity and promote an accountable and transparent process as well as ensuring it runs efficiently. As public sector recruitment can be slow there is a risk of losing good digital talents to faster-moving employers. This means running fast selection ensures that the best candidates are still available when the offer comes. 
Box 5.2 discusses the benefits for the Pennsylvania state government of going from a months-long, paperbased recruitment process to one administered online instead (Ramsey, 2020[71]]).

\section{Box 5.2. Pennsylvania state government recruitment process}

Applying for a job with the Pennsylvania state government used to be a daunting process. Jobs posted had vague, bureaucratic titles such as "Administrative Officer 1." Applicants had to take written exams at a testing centre. Some waited months for a civil service commission to respond by mail before they could interview. Many had moved on by then. Recruitment was thus a very slow and painful way of hiring and wouldn't necessarily get the person with the right skills.

In early 2019 , state lawmakers agreed to streamline the 1940s-era system. Now, the agency oversees a centralized website, where job seekers apply for positions that are more clearly defined. Testing and scoring is folded into the online application process, which administrators track closely.

For corrections officer positions, which required candidates to take written examinations until recently, the change had an immediate impact. The number of applicants tripled within the first week of removing that from a test centre environment to applying online for the job.

Source: Ramsey, Mike (2020[71]), Hiring Challenges Confront Public-Sector Employers.

The public sector can be harmed due to certain restrictions inherent to their recruitment policies. In some countries, the hiring of certain profiles requires an associated academic degree. For example, in Chile the remuneration system gives a "professional bonus" that can be near $25 \%$ of the salary for officials who have an academic degree that lasted over 8 semesters. This generates a bias in favour of formal education and means those who do not meet the criteria receive a considerably lower salary, making the public sector an unattractive employer, especially compared to the private sector without similar constraints on recruiting those with in-demand digital skills.

\section{Recruitment selection}

Public sector teams need to be diverse in order to reflect the society they serve. As each person has a different problem-solving approach based on their background, personal experience and personality, the more diverse a team is, the better it is equipped with problem-solving abilities. The study of Phillips, Liljenquist and Neale demonstrated that by simply adding one "outsider" to a team increases their performance as they spent more time considering other options compared to a homogeneous team $\left(2008_{[72]}\right)$. One example is Apple's Health application. In 2014, Apple released the Health app which claimed to enable users to "track everything from calories to electrodermal activity to heart rate to blood alcohol content to respiratory rate to daily intake of chromium" but omitted to track menstruation (Eveleth, 2014[73]). This exhibits that the majority of tech products are designed and produced by men, which explains why most products prioritise men over women (Sullivan, 2014[74]). As a result, diverse teams cover diverse points of view, details and factors that more homogeneous teams may miss.

A step like this could have helped the United Kingdom's Home Office passport service avoid discriminating against darker-skinned people when they submitted their passport photo. Although the automated check was intended to detect poor quality pictures, BBC research found that images of darkskinned women, were more than twice as likely to be graded low quality compared to light-skinned women (Ahmed and Leslie, 2020[75]). This incident reflected a lack of diversity in the data used to train the facialrecognition systems - training data featuring fewer women or people of colour will not be able to recognise those groups of people as well. This would be less likely to happen if the team was more diverse and included women of colour to consider those points. 
Artificial Intelligence $(\mathrm{Al})$ is an attractive tool for reducing time and cost but caution should be used in embracing predictive hiring tools for selecting the "right" candidate. In 2014 Amazon deployed Al to automate its recruitment but after a year identified that the system was not rating candidates in a gender neutral-way and was in fact discriminating against women. This was because it had been trained with data reflecting the company's historic hiring practices, during which time men had dominated (Dastin, 2018[76]). To get the best candidates based on merit, it is important to ensure that the use of Al for recruitment is based on a foundation of ethical practice and complemented by active efforts to mitigate the bias in any training data and minimise algorithm bias (World Economic Forum, 2019[77]; OECD, 2019 $9_{[58]}$ ).

In order to build diverse teams there needs to be a diverse pool of potential candidates with the suitable skills. These previous examples may result from a lack of women equipped with digital skills, or enrolled in STEM or ICT-related studies and occupations. The OECD Pursuit of Gender Equality report shows that differences in the careers of male and female workers can originate early in teenage years, when choosing the field of specialisation in education. At 15 years of age, on average across OECD countries, only $0.5 \%$ of girls wish to become ICT professionals, against $5 \%$ of boys, and twice as many boys as girls expect to become engineers, scientists or architects. These gender-specific expectations about the future profession exist in 15 year-olds independently of their success in related subjects at school (OECD, 2017[78]), which translates into very few women entering the labour market as ICT specialists and in women representing a very low share of such workers. To address this pressing need, Estonia has developed an innovative initiative, discussed in Box 5.3 with a focus on increasing the share of women among ICT sector students and employees.

To stay competitive, the public sector needs to hire the best candidates according to a merit-based approach and to mitigate any entrenched cognitive biases in the process. It is the responsibility of organisations to make a conscious effort to minimise bias through blind recruitment, the content design of job adverts and the choice of where to advertise. A further mechanism for helping to guarantee equal opportunities for all could be in creating dedicated roles to consider diversity and inclusion, such as that found in the United Kingdom, which has a Digital, Data and Technology Diversity and Inclusion Lead operating on behalf of the Profession from the Government Digital Service as well as similar roles in organisations themselves. This can also be achieved by having laws that promote gender equality such as Portugal (Box 5.4). These examples recognise that actively promoting diversity and inclusion can help create the right environment for digital transformation.

Table 5.1. Attracting the talent for a digital workforce

\begin{tabular}{l|l}
\hline What supports digital transformation & What doesn't support digital transformation \\
\hline Establish clear job description and profile needed & Unclear about jobs and roles the organisation needs \\
\hline Proactive recruitment strategies & Bureaucratic and slow recruitment methods \\
\hline Promote public sector as an employer & No branding strategy \\
\hline Put measures in place to reduce biases in recruitment & Unconscious biases in recruitment \\
\hline Ensure diversity and gender equality of teams & Little consideration of diversity and gender equality of teams \\
\hline
\end{tabular}




\section{Box 5.3. Estonia's nudging methods to increase the share of women in ICT professions}

In 2019 Estonia started an 18-month research project led by the Ministry of Social Affairs concentrating on developing and piloting nudge methods to increase the share of women among ICT sector students and employees. The project is co-funded by the Ministry of Social Affairs and the Estonian Research Council. The following actions are a part of the study:

1. Compiling the current state of play based on existing studies and analysis (including educational choices of girls and women, dropping out of education in the ICT sector, progress in the job market, etc), mapping the possible reasons for the low number of women in the ICT sector. A qualitative study within main stakeholder groups will be carried out.

2. Presenting proposals for nudging methods with the goal of increasing the number of women in ICT, including in management. These methods need to be piloted for at least 9 months.

3. After the pilot phase carrying out an analysis of the implementation of nudging methods as the basis for a final report and recommendations about future use of nudging methods.

Source: Provided by the Working Party of Senior Digital Government Officials (E-Leaders) Thematic Group on Digital Talent and Skills

\section{Box 5.4. Legislation promoting gender equality in Portugal}

Between 1997 and 2017, Portugal had five national plans for the promotion of equality and citizenship:

I. Global Plan for Equality of Opportunities 1997-1999

II. National Plan for Equality 2003-2006

III. National Plan for Equality - Citizenship and Gender 2007-2010

IV. National Plan for Equality, Gender, Citizenship and Non-Discrimination 2011-2013

V. National Plan for Equality: Citizenship, Gender and Non-Discrimination 2014-2017.

Although Portugal's position is still below the European average, these policies have allowed that in recent years Portugal has seen the highest growth rate, compared to other EU countries, having risen 7 places in the Gender Equality Index in 12 years. Several strategic areas have been pursued, with tangible results, namely at legislative level.

One of the laws with most positive impact, in area of equality between women and men, was law $62 / 2017$, which defines minimum thresholds of women and men in boards of public companies (33\% as of 1 January 2018) and listed companies (20\% as of 1 January $2018 ; 33 \%$ as of 1 January 2020). This law has raised the number of women in the boards of listed companies from 12 to $18 \%$, in State companies from 28 to $32 \%$ and in local public companies from 20 to $32 \%$.

Source: Provided by the Working Party of Senior Digital Government Officials (E-Leaders) Thematic Group on Digital Talent and Skills 


\title{
Retaining talents
}

\author{
Retaining the talent for a digital workforce ensures that they stay \\ motivated, their career progressions are clear and that they offer \\ transparent reward systems to keep staff satisfied, improve well-being \\ and decrease turn over.
}

The ability of an organisation to keep its employees, its employee retention, is a critical issue. Although the public sector may be able to offer high job security, retention of digital talents remains a challenge as the private sector can offer higher financial rewards for the same roles and skills. There are significant costs associated with high levels of staff turnover not only through the time implications of recruiting and training new talents but in the loss of institutional knowledge and continuity. Furthermore, low rates of employee retention are indicative of broader organisational malaise that require addressing - if hiring managers wait for the exit interview to understand why staff have chosen to leave the organisation, it is too late. This can disturb the performance of the organisation as it can increase difficulty to retain other talents, as research shows that quitting is contagious (Felps et al., $2009_{[79]}$ ). According to the Society for Human Resource Management, a focus on the retention of staff is fundamental in attracting and retaining valuable talents, reducing cost and increasing productivity (2020[80]).

Retention starts from recruitment. As seen in the previous section, fairness and transparency are both powerful concepts that can make a lasting impression on talents and their retention. Unlike in the past, where employers would expect employees to be 'generalists' and interchangeable public servants across all fields, there is no longer such "one size fits all" approach in recruiting in the public sector given the specific skills and candidate profiles that are required to carry out digital work. Therefore, to retain employees, employers need to focus on what employees want. The discussions of the OECD's Public Employment and Management Working Party have borne out research indicating that candidates are selective in their choice of employer and value purpose, professional development, coaching and a healthy work-life balance when deciding where to work (Gallup, 2016[31]).

Providing clear career planning based on capability-based progression is fundamental in employees' retention. It can be expressed in terms of professional and personal development through training, variety of work and new challenges or regular opportunities for job growth rather than hierarchical promotion, which make employees feel that they are valued and that the organisation cares about their professional and personal development and well-being. In Uruguay, the National Agency for e-Government and Information Society (AGESIC) encourages the retention of its staff in several ways including creating a flexible working environment, providing attractive training in tools and vanguard technologies and offering staff the opportunity to professional challenges of working on projects with national impact. This collectively creates a powerful reason for employees to stay with the organisation.

Given that public sector compensation is not always as financially generous as the private sector it is important to have a clear, transparent and standardised way of compensating talent in order to maintain trust and fairness. In the United Kingdom, pay is locally determined by each department but under the Digital, Data and Technology (DDaT) Professional Capability Framework (discussed earlier in Box 5.1) efforts are being made to create greater coherence in terms of reward for these roles. The DDaT Pay Approach aims to provide simple, effective, and consistent guidance on pay to work with existing flexibilities in an organisation's pay structure to attract, recruit and retain these specialist skills. 
Table 5.2. Retaining the talent for a digital workforce

\begin{tabular}{l|l}
\hline What supports digital transformation & What doesn't support digital transformation \\
\hline Well-designed, fair, trusted and attractive reward system & Rudimentary, unfair and opaque reward system \\
\hline Clear career planning & Unclear career planning \\
\hline Focus on job growth, profession and personal development & Focus on job titles and position in a hierarchy \\
\hline
\end{tabular}

\title{
Developing and maintaining skills
}

\section{Developing and maintaining the skills of a digital workforce involves equipping the public sector with the right skills where possible to reduce dependency on external third parties. Although skills can be developed formally, the workforce also needs to have the incentives to explore informal, flexible, and open peer to peer approaches.}

\begin{abstract}
After hiring and retaining a talented workforce, it is then vital for the public sector to help the workforce to maintain flexibility and ongoing levels of knowledge by developing skills in both formal and informal ways. Establishing a learning culture as discussed in chapter 2 thus became crucial, as it is important that this is not limited to a focus on the work of any central organisation or distribution of talents but that the public sector can approach skills in a coordinated way that can avoid the creation of silos or duplicate efforts without limiting the agency and autonomy of public servants to experiment and learn.
\end{abstract}

\section{Formal training}

Formalising training by making it mandatory for all public servants is a clear indication of an organisational commitment to build a learning culture and invest in the knowledge of staff. If training remains informal, there is a risk that it is not taken seriously and, as a result, is not implemented. In Spain, the public administration institute is responsible for formalising the training of public servants and preparing the General Strategic Plan every four years with each Ministry developing its own sectoral training plan, based on the needs of its employees. Italy has a similar initiative for all public servants called "Digital Skills for the Public Administration" (see Box 3.4). The Ministry of Information and Communications Technologies (MinTIC) of Colombia, through the Digital Government Directorate, also recognised the need to develop skills to support different areas of Digital Government policy across the government. Consequently, their project planning provided funding to carry out training on digital government topics such as usability, open data and data science, IT architecture, digital security, and in particular emerging technologies. Investing in formal training embeds digital skills as part of the standard operations for the public sector on an ongoing basis and cultivates a sustainable learning culture.

Such formal training programmes can be extended beyond public servants to promote and develop a learning culture in society more broadly. For example, Uruguay has several public bodies targeting different segments of society. AGESIC offers tailored training to meet the needs of particular segments, for example, on cybersecurity for the financial sector. Plan Ceibal (One laptop per child Program) focuses on digital skills for children and teenagers and is complemented by the "Young People Programming" scheme offering training in software testing and development. These programmes aim to familiarise society 
with the opportunities and challenges of technology and facilitate the use and implementation of digital public services. In Estonia, the government identified digital technologies as a priority for economic growth and has worked with universities and the IT industry since 2012 to secure the necessary workforce for the ICT sector. To enable the shift to digital at a country level, organisations need to take the initiative to establish $21^{\text {st }}$ century skills for all. Schools and universities are critical in this process as they train the workforce of tomorrow but so too is the private sector in communicating their needs and challenges so that young people can be equipped with the skills to match the demand.

Once training is formalised, it needs a variety of appealing content matching the strategy and objectives for digital transformation. Patall, Cooper and Robinson (2008[81]), showed that providing multiple choices can create intrinsic motivation and therefore organisations need to understand how their employees prefer to learn as well as generating appropriate and useful content. "Push" based training, meaning employees learn through lectures and pre-determined curricula (Prestera, 2015[82]) has been criticised as too rigid and passive with the result that it fails to engage employees in their own professional development causing low satisfaction, high rates of dropouts and complaints that training has a low impact on improving performance (Prestera, 2015[82]). Instead of being told what and when to learn, today's learners want access to userfriendly learning material and acquire skills on demand, known as "pull" based learning (Prestera, 2015 ${ }_{[82]}$ ).

Employees need to see the value of what they are learning in order to be actively engaged, empowered and motivated in the learning process and consequently upskill, achieve better results and generate higher satisfaction towards their job and employer. Making the shift from push to pull learning, so that learning becomes a continuous process instead of a series of trainings, involves promoting new learning methods, cultivating a curious and growth mindset (Dweck, 2006[83]), creating a feedback loop and iterating content to make it fit current needs. To strengthen the growth mindset in organisations, it is critical to give meaningful and constructive feedback as well as rewarding staff that have successfully learned new skills or capabilities, so that other staff are inspired to follow their example.

In addition to hiring new staff and formalising training to increase the skills of those within a public sector organisation, there is a need to access skills and talent in other ways. Consultancy services, whether through an internal resource or procured externally can be one route to solving a particular problem or addresses a specific skills gap. The challenge is for the impact of this additional resource to be long lasting as once consultants are gone, the organisation or team can easily revert to its old ways of working. One way of mitigating this risk is to ensure that any consultancy work involves creating an internal exchange of expertise and explicit skills transfer to reduce dependency on third parties. Holistic digital government strategies and far-sighted leadership play an important role in understanding how to balance the skills already available to an organisation with the resources it can acquire.

Internal consultants operating as a resource of public servants can play an important role in providing advisory capacity and supporting effective change management and implementation in the public sector. Using their consulting expertise they can help to improve organisation performance, work within the organisational structure to solve issues and implement more efficient solutions, serve as a facilitator or act as an agent within an organisation. For example, public servants working for $18 \mathrm{~F}$ in the United States of America or operating within Canada's Free Agent Model (Box 5.5) are tasked to help, equip, analyse and assess the balance between commissioning an external consultant to help solve the problem, getting coaching and solving the problem internally, or recruiting new talents in the long run. These internal strategic responses require public servants to understand the dynamics of the sector and the organisation, as well as core consulting skills to best lead, implement and transfer the right change and skills. 


\section{Box 5.5. Canada's Free Agents program}

Canada's Free Agents program was launched in 2016 as a new model for workforce mobilization. It offers public servants the freedom to select work that matches their skills and interests and allows them to make a contribution that they find meaningful. It also supports managers looking to rapidly and easily acquire top talent with emerging and core skills to support their short-term project needs. Free Agents are screened for attributes that are beneficial for solving problems and skills that are in demand.

Source: Provided by the Working Party of Senior Digital Government Officials (E-Leaders) Thematic Group on Digital Talent and Skills

External, private sector, consultants can be an important partner for governments in developing their capacity to deliver on their ambitions for digital transformation, particularly during early phases of establishing a core competency for digital government skills. In the United Kingdom this process was supported by developing the Technology Code of Practice discussed in Box 5.6 to set expectations for working with suppliers that maintained high quality outcomes, encouraged skills transfer to public servants and retained access to data without losing ownership of intellectual property. Colombia and Spain have also prioritised knowledge transfer through contractual clauses that ensure public sector employees are trained in specific skills by suppliers. Uruguay also has contractual clauses that determine the ownership of the resulting products, as well as provision for knowledge transfer and its evaluation. Recognising skills transfer as a formal part of the commissioning process is valuable for seeing public sector workforces retain knowledge and develop the skills to solve similar or upcoming issues on their own.

\section{Box 5.6. The United Kingdom's Technology Code of Practice}

The Technology Code of Practice is a tool for all technology projects or programmes. It recommends public servants to consider each point, align project or programme with the mandatory points, and follow as many of the remaining points as is practical. The more organisation's technology and business strategies align with the Technology Code of Practice, the more it benefits from it.

Following the Technology Code of Practice helps introduce or update technology so that it:

- $\quad$ meets user needs, based on research with your users

- $\quad$ is easier to share across government

- $\quad$ is easy to maintain

- $\quad$ scales for future use

- $\quad$ is less dependent on single third-party suppliers

- $\quad$ provides better value for money

The Technology Code of Practice contains guidance and case studies to help public servants migrate from legacy infrastructure and manage the full lifecycle of their technology.

Source: Government Digital Service (2019[84]), Technology Code of Practice

Similarly, skills transfer is the idea that the person transferring the skill needs to understand, explain and demonstrate the particular skill to their peers and practice with them until they can reproduce it. For example, in Slovenia, knowledge transfer between outsourced contractors and public servants is done in different ways either by participating in different workshops or collaborating in work-related activities on a 
daily basis. When outsourcing certain projects, such as the development of a new information system, a new model or a digital tool, etc., it has been agreed with the outsourced contractor that they should carry out various trainings for public servants, who will encounter these newly developed instruments while performing their job and will need to be able to use them accordingly. This proves that the person teaching does not need to be a professional trainer but can simply be an employee with a particular skill that they want to share with their peers to fill a skill gap that has been identified.

\section{Informal training}

Besides these formal activities, the public sector needs to be flexible and open to collaboration. It should encourage individuals to reach out to one another and build connections between peers to create a more organic organisation. Although it is vital to put specific training activities in place to secure and reinforce digital government, public sector organisations must also value implicit knowledge and learning from ongoing experience. Although this reduces control over the content of what is learnt and reduces structured expectations of what individuals should learn, it generates a soft and peer-to-peer dynamic rather than an organisation-to-employee dynamic, which is usually more supervised.

One way of helping digital talents to maintain, reuse and exchange the knowledge they have acquired is through communities of practice, which are "groups of people who share a concern or a passion for something they do and learn how to do it better as they interact regularly" (Wenger-Trayner and WengerTrayner, 2015[85]). Reflecting the learning culture addressed in chapter 2, this approach creates a safe environment where people can grow, experiment new things and learn from and with each other. This informal activity is fundamental to good management of knowledge as sharing knowledge is a social process, making learning and communities inseparable (Ceptureanu and Ceptureanu, 2008[86]). This learning practice not only gives individuals a social context to which they belong while learning with and from but also helps organisations to develop and sustain knowledge. Members of these communities share skills and expertise develop in their day to day work and decide on useful topics they can address together. As an example, AGESIC in Uruguay has established several communities of practices to develop and sustain the necessary skills in fields including cybersecurity, organisational change management, project management, and enterprise architecture. This shows that communities of practice can be made of a variety of topics and create a safe space for members to develop and exchange skills and knowledge, which facilitate digital transformation.

As part of being agile, sharing experiences and reflecting within the organisation are important practices. Show and tells, as mentioned in chapter 2, allow teams to articulate their success and identify lessons learned. A further technique, the blameless retrospective - a regular "improvement" meeting held to find ways and means to identify potential pitfalls, past mistakes, and seek out new ways to do better - is valuable for reflecting on what was successful and what could be improved in the future while establishing a climate of mutual trust and respect.

A workplace with an environment that fosters the sharing of experiences and information can be powerful for building momentum amongst practitioners and reducing silo mentalities at individual, team and organisational level. An organisation where teams or individuals withhold information from others for reasons that may include fear, power struggles, organisational inefficiency or the lack of mechanisms for sharing and an absence of effort to establish them, is not a healthy environment. Siloed practices break trust and reduce communication with negative implications for organisations' productivity, budget and time.

Replacing a silo mentality with an expectation of sharing and collaboration within an organisation can improve performance and comradery while cultivating a work environment based on trust and communication. This echoes Chapters 2 and 3 which discussed the role of leaders to encourage the sharing of practices, organisational structures to cultivate communities or groups to promote collaboration, and life-long learning to strengthen the work culture and image of the public sector as an employer. 
However, the challenge many organisations face is how to incentivise people to do things that they want them to want to do, rather than things that are already part of their job description. Given that informal skills development requires actions, planning and motivation from the workforce to make it happen, it is crucial to find a way that encourages the workforce to make these efforts voluntarily. When Microsoft acquired Yammer in 2012, they saw an opportunity to improve team dynamics by incentivising staff to share more by making it a measure of their performance (Branscombe, 2015[87]). Incorporating a tool, Yammer, into performance measurement, helped increase the company's culture of collaboration.

\section{Table 5.3. Developing and maintaining the skills of a digital workforce}

\begin{tabular}{l|l}
\hline What supports digital transformation & What doesn't support digital transformation \\
\hline Formal training available & No training available \\
\hline Pull learning & Push learning \\
\hline Consultancy and skills transfer to build internal knowledge & Dependent on the knowledge of external third parties \\
\hline Informal means of support & No informal means of support focusing on digital \\
\hline Share good and bad experiences/retrospective meetings & Keep experiences for themselves/work in silos/no reflection \\
\hline Sharing and collaborative culture & Silo culture \\
\hline
\end{tabular}

\section{Allocating talents and skills}

\section{Allocating the talents and skills within a digital workforce involves making sure organisations can access the right people for the right roles. This can be done through job mobility for employees to exercise their autonomy by working on topics that interest them and through deploying staff to work as part of multi-disciplinary teams that work across organisational siloes.}

In light of digital transformation being built on teams' performance, organisations need to consider a structure that can bring the best out of the talents they hire and empower them to achieve high performance. An efficient allocation of people with the right skills to the right task not only boosts productivity, but also employees' satisfaction. This can be done through the opportunity to change jobs within the organisation according to one's interest, mentoring programmes, switching from funding projects to funding people and incorporating feedback loops.

To empower and motivate all staff, public sector organisations should offer their employees the opportunity to work on projects related to their personal or professional interests through internal job mobility of inhouse talent into roles for which they are better suited. Giving public servants this opportunity can create higher motivation and a happier workplace, which will benefit organisations through increased performance and improved staff retention. As well as increased job satisfaction, job mobility within an organisation can allow individuals to feel empowered in their career advancement. In Portugal, mobility is a legal 
mechanism originally established as part of efforts to safeguard the rights of employees that were left without work because of organisational restructures. As a result, Portuguese public employees can temporarily perform the same or different functions within the same organisation, or elsewhere, without the need for a recruitment procedure. In Uruguay, AGESIC's role in working with different public sector organisations means public servants and consultants adapt to different scenarios and needs according to the project they're involved with. Job mobility programmes can help retain talents, create career growth opportunities and promote continuous learning, adaptability and well-being but these opportunities need to be well-designed and allocated in order to have a real impact on digital government.

Given that bringing together different professions fosters innovation, enables the sharing of perspective and improves team delivery, forming multidisciplinary teams must be a priority when distributing skills. Multidisciplinary teams enable the generation of broader ideas, which facilitates learning with and from each other. These teams need to reflect not only a mix of professional skills but also a varied socioemotional makeup and diversity of background and need to be supported by a strategic approach to job families, descriptions and allocation that is intentional in forming teams rather than treating individual professions as isolated functions.

The funding available for digital government is an important element of digital transformation. One approach is to providing funding to projects, with funding ending when a piece of work is defined as finished. Agreeing on a budget, a project and a timeline is argued to give people certainty, the ability to predict and set up a strategy to achieve a goal, while assuming that nothing will change (Thomas, 2017[88]). However, this can create the false certainty that a particular area of focus will no longer need investment, more typically however, at the end of the project, additional investment is required to maintain and continue meeting the needs identified for the service, product of value stream.

As events associated with the COVID-19 pandemic have shown, public sector organisations have to react to a constantly changing world in which things move quickly. As a result, project-based funding may not be flexible enough to adjust to situations. The alternative approach to funding is to focus on funding the professional and personal development of teams to maintain services, products or value streams. By approaching funding in this way it allows organisations greater flexibility to adjust their priorities, support digital infrastructure (such as hosting, identity and other common components) as 'products' and to increase the numbers of public servants employed instead of relying on contractors (Thomas, 2017 $[88]$ ). To enable greater flexibility, organisations can reduce projects into smaller discrete experiments (or tests) that allow hypotheses to be tested with real end users (Commonwealth Government of Australia, 2019[38]). This way of allocating funding to teams to acquire the right skills contributes to making the work environment more receptive to digital transformation as it invests in the growth of public servants and their engagement in the long run to develop great services.

In addition to this, to ensure talents and skills are well assigned, public servants should be able to meet with their managers on a frequent basis, set achievable objectives and establish a 360-degree feedback loop culture to ensure they have the support they need to complete their tasks successfully. In Slovenia, enshrines this activity into law with the Public Employees Act requiring superiors to monitor the work, professional qualifications, and careers of public employees with at least one annual interview with each public employee during which the need for any training is discussed to improve their work performance, professional knowledge and career development. Spain similarly monitors the performance of public servants through an annual report looking at the activities of the previous year, which then forms the basis for identifying future goals. These efforts help managers to understand the performance of individual talents and identify issues that need to be addressed, which carried out systematically helps to improve productivity in the organisation as a whole.

An important addition to performance management are mentoring programmes that help guide employees in developing new skills and exploring new opportunities within an organisation. Mentoring programmes pair mentors with mentees and create opportunities for transfer of skill, knowledge and experience between 
staff. These pairs can be between established and new, high performing and under-performing, or senior and junior (as well as reverse mentoring from junior to senior). Acting as a role model, mentors often help mentees integrate in the organisation and share learning experiences. This creates an opportunity for the mentor to give back to the organisation by training staff and using their experience to help mentees succeed in the organisation. For mentees, this is the chance to get familiar with workplace routines, policies and expectations, and broaden their network, which enable them to feel included and foster their sense of belonging. As for the organisation, mentoring programmes create in-house training, help new hires develop good habits and cultivate loyalty.

Table 5.4. Allocating a digital workforce

\begin{tabular}{l|l}
\hline What supports digital transformation & What doesn't support digital transformation \\
\hline Job mobility & Fixed structure \\
\hline Multidisciplinary teams & Homogenous teams \\
\hline Funding teams & Funding projects \\
\hline Regular feedback loop & Little feedback from experienced staff \\
\hline Mentoring programme & No guidance \\
\hline
\end{tabular}

\section{Reforming the environment}

\section{Reforming the environment in which a digital workforce operates involves the rapid adoption of new ways of working. Organisations need to reimagine a work life that is flexible and agile to be prepared for future crisis but also to create the conditions for an increasingly digital-first workforce to feel safe and stay productive.}

The COVID-19 pandemic will be remembered for many reasons, mostly negative. In the context of digital transformation, it has given a great push to organisations to rethink their working environment to enable the creation of a digital workforce and in parallel prioritise the recruitment and retention of a digital workforce to build an environment to result in digital transformation.

As the work environment and a digital workforce need to complement each other, organisations rethinking job families to reflect the discussions in Chapter 3 should ensure attractive career pathways. This model can manage performance and expectations, which helps create more consistent performance measures and a clearer career roadmap. This can also boost recruitment levels by attracting potential candidates as they recognise a solid career development even into areas of skills shortage.

Organisational structures that promote life-long learning culture and provide training to a digital workforce build incentives to lead informal peer-to peer activities, participate in mentoring programmes and ask for regular feedback. These measures not only make sharing, collaborating and learning from each other a norm, but also create an environment that is safe enough for staff to admit failures. While experimentation is essential for innovation and ongoing improvement within an organisation, the COVID-19 pandemic has 


\section{4 | GOV/PGC/EGOV(2020)9}

shown how important the skills and capacity for experimentation are for finding the best ways to work in fully remote or increasingly hybrid configurations.

Since the world is moving towards ways of working that encourage greater agility, are less hierarchical, offer autonomy and increase transparency it is important for public sector organisations to develop HR policies that support greater flexibility and corporate support for the necessary software tools or physical material to improve home-based working environments. Some organisations are providing financial support to cover the costs incurred from employees' working at home (desks, chairs and computers) as well as regular allowances for WiFi and telephone costs. For instance, Twitter announced that it would give its teleworkers $\$ 1,000$ to set up their new home office and Buffer, a software application company, which became fully remote in 2015, pays its employees' internet bills. Each employee also gets a sum of $\$ 200$ for technology costs, and $\$ 500$ to set up their home office (Nova, 2020 $[89]$ ). These examples prove that digitally-enabled and flexible organisations can keep productivity and satisfaction high, promote the image of an attractive employer that cares about well-being, build trust and thus greatly contributes to retention of their workforce.

The COVID-19 pandemic forced public sector organisations to change their working practices, almost overnight and relied on the development of staff culture and practices to enable fully remote, hybrid or inperson working environments. As the world looks to a future informed by the experiences of COVID-19, public sector organisations will have decisions to make about how best to model their work environment and employment structures; they will have to re-evaluate how work is done, whether work goes to people or people go to work and invest in offices and physical workspace that are conducive to digital ways of working. In doing so, they will need to ensure that they have a vision for developing a digital workforce, capable of instigating and maintaining a digital transformation that delivers on the potential of digital government, as well as room for the creation of new job profiles that do not yet exist to attract and retain talents and at the same time to support better policies for better lives. 


\section{Conclusion}

The world is increasingly finding that digital has become the default with the use of the Internet and digital technologies affecting almost every aspect of people's lives - from how they live to how they work. Almost all occupations need to adapt not only to the ubiquity of the Internet but to the presence of different digital technologies by either upskilling or reskilling through training in order to take on new and different roles. The public sector is no different and needs to embed digital in every role of the organisation structure.

Embedding digital requires an evolution from the approach and concerns taken to establish e-government. Although the use of information and communications technology to improve government has helped to reduce costs, improve services, save time and increase public sector effectiveness and efficiency, egovernment practices also tended towards creating a discrete profession working separately from other professions with the responsibility for implementing and managing ICT. Digital government maturity requires different skills and different cultural approaches with it being a priority for those working on capability in the digital era to avoid creating silos and ensure these opportunities and knowledge are broadly embedded across the public sector, no matter the role or responsibility.

The framework presented in this Working Paper has addressed the individual and team-level opportunities in the context of developing digital government maturity. The framework is divided into three pillars.

\section{Pillar 1: The right environment to encourage digital transformation}

The first pillar (chapter 2) identifies the characteristics of a successful digital enabling environment, which includes strong leadership, flat organisational structure, digital job families, life-long learning culture and new ways of working.

Leaders must have a clear vision of the role of digital and actively champion the benefits of digital government. This will help set a digital culture within the workforce and the organisation. For this, they need to build solid knowledge and skills about the assets of digital transformation and efficiently communicate the strategy to achieve it. They should demonstrate their engagement in the organisation's work, which will not only enable leaders to better understand their current activities and better shape an achievable vision, but also motivate the workforce.

Effective leadership behaviours will be supported by reducing layers of hierarchy and delegating decision making. By doing so, leaders will strengthen trust from the workforce and increase productivity, as well as job satisfaction. Leadership teams can decentralise decision making to empower their workforce and create flatter organisations that are more flexible and faster-moving in responding to the needs of citizens.

A further element in encouraging digital transformation is recognising that the evolution of public service design and delivery requires a focus on establishing digital professions with objectives and roles that are user-centred and rooted in digital government practice. Organisations need to stay alert for job profiles that need to be created, revise their job families and the way they describe the roles within them to create teams that are better suited to delivering timely, trustworthy, proactive and data-driven services that are built around a deep understanding of their users' experiences.

It is crucial for organisations to establish a learning culture that encourages and provides safety for employees to experiment. Leaders can create an environment of trust by encouraging and incentivising 
employees at all levels to take time to learn and share, take risks and allow mistakes through experimentation, mentoring, communities of practice, show and tells and retrospective meetings. This will not only enable innovation of products and services but also enrich peer learning and personal growth, further contributing to job satisfaction.

By supporting different ways of working with necessary policies, tools and technologies, organisations become more flexible and adapt to the needs of their talent. Working environments like these are vital to support the growing preference for Agile and user-centred design methodologies in pursuit of quality public services. Offering digital talents the opportunity to work in different ways makes a further valuable contribution to trust and satisfaction.

\section{Pillar 2: Defining skills for a digitally enabled state}

The second pillar (chapter 3 ) recognises that in order to create a 'digitally enabled state' it is important to develop a holistic approach to the skills of public servants that recognises digital government is core to that experience, regardless of their role or position in the organisation.

The starting point for any workforce are the skills of society making societal-level 21 st century skills the critical foundation to any skills work in any domain. It is important to ensure that society as a whole is capable and equipped with the necessary skills to thrive in the digital age. All efforts to establish a digitally enabled state rely on a grounding for all to have basic confidence in using digital tools and technologies.

For public servants the expectation needs to go beyond the societal baseline for $21^{\text {st }}$ century skills to recognise digital government user skills as a core part of their contribution. All public servants need to be equipped with the skills that are supportive of digital government maturity, which includes Recognising the potential of digital for transformation, Understanding users and their needs, Collaborating openly for iterative delivery, Trustworthy use of data and technology, and Data-driven government.

In order to realise the full benefits of digital transformation, it is not sufficient to develop digital and data literacy and cognitive skills, attention must also be given to digital government related socio-emotional skills too. Unlike digital government user skills, every employee doesn't need to master each of the digital government socio-emotional skills and their associated behaviours, instead organisations need to ensure that they are reflected at a team level when products and services are designed and delivered.

The diversity of teams should not only reflect this variety of socio-emotional skills but embrace a multidisciplinary approach that ensures the perspective of a variety of professionals - digital and non-digital - to support digital government maturity. Digital professions include User-centred design, Service, Product, Data, Delivery and Technology role each of which warrants access to the right digital professional skills.

Leaders play a significant role in modelling digital government user skills and shaping the environment that encourages a digitally enabled state. It is crucial that organisations ensure their leaders have the skills to oversee digital transformation, are informed and aware of the benefit of going digital and can communicate the purpose and vision to build momentum and on board the organisation in its journey.

\section{Pillar 3: Path to a digital workforce}

As seen at an organisational level, the third pillar (Chapter 4) applies the activities identified by the OECD's Skills for a High Performing Civil Service report (OECD, 2017[13]) to building a digital workforce for digital government, namely attracting talents and skills, developing a retention programme and allocating skills informally.

To promote the public sector as an attractive, trustworthy and transparent employer, a proactive recruitment team and strategy must be in place. This will help communicate the recruitment process, 
recruitment selection and benefits of working in the public sector. Organisations need to ensure recruitment is well-designed to attract the right candidates in the first place and take an approach to testing the necessary skills for the position that is merit-based, inclusive and reduces delays.

Organisations must also set up a well-designed, fair, trusted and attractive reward system with clear career planning to help retain their workforce. This will give clarity to talents' career evolution, increase transparency and motivate them to grow by giving them a concrete objective in mind. One of the opportunities available to the public sector is to emphasise the values-driven nature of their work and motivation for their workforce meaning reward systems can be designed around more than financial reward.

Managers are encouraged to prioritise job growth and professional development through the set-up of multidisciplinary teams. Employees should have the opportunity to offer and grow their expertise by collaborating with different teams from different departments. This way, they can deepen their knowledge in a specific field by moving around the organisation to enlarge their vision.

In addition to this, organisations need to invest in digital talents by providing appropriate training that can be formal or informal in nature and benefit from both generic and bespoke guidance. It is essential that organisations pay attention to employees' needs, support and guide them in their personal growth and satisfaction. This can be done through formal training, peer learning, communities of practice, as well as regular feedback loops and mentoring programmes.

To satisfy personal interest and retain their digital workforce, organisations should also offer job mobility by providing a variety of career choices. This gives the chance to employees to explore other positions, diversify their knowledge and earn different expertise, which contributes to increasing their well-being within the organisation and thus their retention.

This Working Paper maps out the different pillars that organisations need to consider when committing to a digital transformation. However, further work is needed to operationalise certain of the elements it contains. Indeed, not only is collaborating with the OECD Public Employment and Management team to explore the development of a common view regarding public sector workforce skills critical, but also in order for governments to establish the necessary skills amongst their public servants to achieve digital government maturity necessitates a model that can scale.

Therefore, drawing on the examples of country practices provided throughout the paper and the ongoing deliberations of the Working Party of Senior Digital Government Officials and its associated Thematic Groups as well as of aligning Human Resources policy with digital delivery, future work could include the development of a toolkit to help individuals and organisations measure their levels of proficiency across the framework. Taking the form of a rubric, a short description of what is expected and examples of applications of such skills could be made available, with a scoring system from basic to advanced proficiency.

\section{Key recommendations}

This working paper shows pathways for developing a digital public workforce with the necessary skills to achieve a successful digital transformation. The following actions are suggested to guide governments in considering the contextual, skills and talent management aspects of digital transformation.

- Building the right environment for encouraging digital transformation requires leaders and their organisations to:

- be aware of the digital skills that a workforce requires to keep pace with digital evolution

- communicate a clear and understandable vision of the role of digital and actively champion the benefits of digital government 
- demonstrate their engagement by visibly endorsing and actively participating in the rhythm of digital delivery, reducing hierarchical layers and delegating decision making by empowering teams as the unit of delivery

- focus on digital professions that are user-centred and have specific objectives and roles

- establish a learning culture that encourages and provides safety for employees to experiment

- support different ways of working with necessary policies, tools and technologies.

- Establishing the skills for a digitally enabled state requires:

- a broader digital skills strategy for society as a whole ensuring all are equipped with the necessary skills to thrive in the digital age

- public servants equipped with the digital user skills that support digital government maturity

- diverse and multidisciplinary teams consisting of well-trained digital and non-digital professionals reflecting a blend of digital government socio-emotional skills and behaviours to design and deliver trustworthy and proactive services with users' needs in mind

- leaders that model digital government user skills and actively shape the environment to create a digitally enabled state.

- Creating a path to a digital workforce requires:

- proactive recruitment strategies that promote the public sector as an attractive, trustworthy and transparent employer

- well-designed, fair, trusted and attractive reward systems that support clear career planning

- managers who emphasise job growth and professional development through multidisciplinary teams

o investment in digital talents by offering regular feedback loops and mentoring programmes, and providing training in both formal and informal ways

$\circ$ encouraging job mobility and a diversity of career choices for public servants. 


\section{Glossary}

\begin{tabular}{|c|c|}
\hline Term & Definition \\
\hline $21{ }^{\text {st }}$ century skills in society & $\begin{array}{l}\text { Society as a whole is capable and equipped with the necessary digital, } \\
\text { cognitive and socio-emotional skills to thrive in the digital age. This is core for } \\
\text { all to have basic confidence in using digital tools and technologies. All efforts } \\
\text { to establish a digitally enabled state rely on this grounding. }\end{array}$ \\
\hline Agile & $\begin{array}{l}\text { A project management methodology based on the Agile Manifesto } \\
\text { (http://agilemanifesto.org/). Originally focused on software development its } \\
\text { values and principles are increasingly being applied to other areas of } \\
\text { government delivery too. Agile is an iterative delivery methodology that values } \\
\text { human communication and feedback, adapting to change, and producing } \\
\text { working results. }\end{array}$ \\
\hline Career paths & $\begin{array}{l}\text { Routes that individuals take which can be expressed in terms of professional } \\
\text { and personal development through training, variety of work and new } \\
\text { challenges or regular opportunities for job growth rather than hierarchical } \\
\text { promotion. }\end{array}$ \\
\hline Coaching & $\begin{array}{l}\text { The training or development that helps an employee achieve a learning goal. } \\
\text { A coach is someone that is already knowledgeable in a particular area or has a } \\
\text { particular aptitude for one to one skills training and helps an individual } \\
\text { achieve their learning goal. }\end{array}$ \\
\hline Community of practice & $\begin{array}{l}\text { A group of people who share a profession and interact regularly in order to } \\
\text { learn from one another about how to fulfil their roles better. }\end{array}$ \\
\hline Competencies & $\begin{array}{l}\text { Competencies are inherent qualities an individual possesses. It is a } \\
\text { behaviour that specifies how an individual carries out the skills they have. }\end{array}$ \\
\hline Digital capability & $\begin{array}{l}\text { Digital capabilities are defined as those which equip someone to live, learn } \\
\text { and work in a digital society, which is a composite of skills, knowledge and } \\
\text { experience. }\end{array}$ \\
\hline Digital government leadership skills & $\begin{array}{l}\text { The leadership to establish a digitally enabled state draws on wider investment } \\
\text { in the general quality of leadership. However, achieving digital government } \\
\text { maturity requires leaders to visibly model digital government user skills and } \\
\text { actively shape an environment that encourages digital transformation. }\end{array}$ \\
\hline
\end{tabular}


Digital government professional skills

Digital government socio-emotional skills

Digital government user skills
The digital transformation has disrupted existing professions and created new ones. Digital government maturity is supported by building multi-disciplinary teams that draw from, invest in and acknowledge both digital and non-digital professions.

Achieving digital government maturity involves championing and ensuring a blend of domain specific socio-emotional skills and their associated behaviours. Striking a balance between vision, analysis, diplomacy, agility and protection is essential to the design and delivery of trustworthy and proactive services that put users at their heart.

Built on competency in society, these are the 5 areas of core skill needed for all public servants to support digital government maturity:

- Recognising the potential of digital for transformation,

- Understanding users and their needs,

- Collaborating openly for iterative delivery,

- Trustworthy use of data and technology, and

- Data-driven government

Digital literacy

The American Library Association (ALA) defines digital literacy as "the ability to use information and communication technologies to find, evaluate, create, and communicate information, requiring both cognitive and technical skills."

Digital mindset

A digital mindset requires us to see old problems and old processes with new eyes. It is a different way of thinking about customers, services, and process. It's faster, iterative, and adaptable.

Digital skills

Digital skills are defined as a range of abilities to use, apply and understand information in a digital society. They enable people to create and share digital content, communicate and collaborate, and solve problems for effective and creative self-fulfillment in life, learning, work, and social activities at large.

Digital talent

In an individual, 'digital talent' is the level of aptitude someone has for understanding, implementing and engaging with digital transformation'. At a corporate level 'digital talent' are the people working to successfully make digital transformation happen.

Digital workforce

Employees of an organisation equipped with the necessary digital skills to contribute and lead digital transformation.

Flexible working

A way of working that suits an employee's needs, for example having flexible start and finish times, or working from home.

Future workforce

Awareness that the workforce of the future will increasingly have to become more agile and challenge the traditional functioning of workplaces. These factors include the increasing casualisation of work, demands for work-life balance, the role of technology, an ageing workforce, legislative and consumer shifts and a generally more uncertain working environment. 


\begin{tabular}{l|l}
\hline ICT skills & $\begin{array}{l}\text { ICT stands for 'Information Communication Technology'. Everyday usage of } \\
\text { digital technology includes when you use a computer, tablet or mobile phone, } \\
\text { send email, browse the internet, make a video call - these are all examples of } \\
\text { using basic ICT skills and technology to communicate. }\end{array}$ \\
\hline Job families & $\begin{array}{l}\text { A series of related job that need to be revised to reflect a user-centric, iterative } \\
\text { and collaborative approach compared to traditional approaches which tend to } \\
\text { operate independently without collaboration or alignment. }\end{array}$ \\
\hline Multi-disciplinary team & $\begin{array}{l}\text { A team that includes a variety of different roles bringing together several } \\
\text { digital, data and technology disciplines. A digital service team might contain: } \\
\text { a product manager, service owner, delivery manager, user researcher, content } \\
\text { designer, designer, developers as well as procurement experts, lawyers, } \\
\text { policy professionals and those involved in operational support. }\end{array}$ \\
\hline Pay \& reward policy & $\begin{array}{l}\text { All the benefits an employer can offer its employees, including financial } \\
\text { benefits such as wages, specialist salary allowances, bonuses, pensions, paid } \\
\text { leave and sick pay; and non-pay benefits such as flexible working, } \\
\text { opportunities for development and ongoing training. }\end{array}$ \\
\hline Skills transfer & $\begin{array}{l}\text { A method by which an employee is taught how to perform a new task or skill. } \\
\text { In the public sector context this can form part of the contract with an } \\
\text { outsourced external supplier to build capacity in order to hand over } \\
\text { responsibility afterwards. }\end{array}$ \\
\hline User-centred design & $\begin{array}{l}\text { The Interaction Design Foundation defines it as an iterative design process in } \\
\text { which designers focus on the users and their needs in each phase of the } \\
\text { design process. }\end{array}$ \\
\hline & \begin{tabular}{l} 
and \\
\hline
\end{tabular} \\
\hline
\end{tabular}




\section{References}

Agile Alliance (2020), Agile 101, https://www.agilealliance.org/agile101/.

Ahmadya, G., M. Mehrpourb and A. Nikooraveshb (2016), Organizational Structure.

Ahmed, M. and D. Leslie (2020), UK passport photo checker shows bias against dark-skinned women, https://www.bbc.com/news/technology-54349538.

Beck, K. et al. (2001), Manifesto for Agile Software Development, https://agilemanifesto.org/.

Blok, M. et al. (2011), New Ways of Working: A Proposed Framework and Literature Review, Springer, http://dx.doi.org/10.1007/978-3-642-21716-6.

Bock, W. (2012), Envisia Learning, http://blog.envisialearning.com/gamification-of-recruiting-thearmy-experience/.

Branscombe, M. (2015), How three years of Yammer has changed Microsoft for the better, https://www.zdnet.com/article/how-three-years-of-yammer-has-changed-microsoft-for-thebetter/.

Burns, T. and F. Gottschalk (eds.) (2019), Educating 21st Century Children: Emotional Well-being in the Digital Age, Educational Research and Innovation, OECD Publishing, Paris, https://dx.doi.org/10.1787/b7f33425-en.

Carrasco, M. et al. (2020), Getting to Agile at Scale in the Public Sector, https://www.bcg.com/enau/publications/2020/getting-to-agile-at-scale-public-sector.

Ceptureanu, S. and E. Ceptureanu (2008), Role of Knowledge Based Communities in Knowledge Process, http://www.management.ase.ro/reveconomia/2015-2/5.pdf.

Chernyshenko, O., M. Kankaraš and F. Drasgow (2018), "Social and emotional skills for student success and well-being: Conceptual framework for the OECD study on social and emotional skills", OECD Education Working Papers, No. 173, OECD Publishing, Paris, https://dx.doi.org/10.1787/db1d8e59-en.

Chetkovich, C. (2003), What's in a Sector? The Shifting Career Plans of Public Policy Students, https://doi.org/10.1111/1540-6210.00330.

Cobbold, R. (2020), Recruit the best digital talent: ten top tips, https://www.michaelpage.co.uk/our-expertise/digital/digital-talent-top-tips.

Collins, B. (2008), What's the Problem in Public Sector Workforce Recruitment? A Multi-Sector Comparative Analysis of Managerial Perceptions, 
http://dx.doi.org/10.1080/01900690802434214.

Commonwealth Government of Australia (2019), Our Public Service, Our Future. Independent Review of the Australian Public Service, https://pmc.gov.au/sites/default/files/publications/independent-review-aps.pdf.

Conway, M. (1967), Conway's Law, https://www.melconway.com/Home/Conways Law.html.

Dastin, J. (2018), Amazon scraps secret Al recruiting tool that showed bias against women, https://www.reuters.com/article/us-amazon-com-jobs-automation-insight-idUSKCN1MK08G.

Digital Technology Hub (2020), Difference between ICT and DT, https://www.digitaltechnologieshub.edu.au/teachers/australian-curriculum/ict-vs-digitaltechnologies.

Dweck, C. (2006), MindSet: The New Psychology of Success, Random House.

European Commission (n.d.), The Digital Competence Framework 2.0 / EU Science Hub, The Digital Competence Framework for citizens (DigComp), https://ec.europa.eu/jrc/en/digcomp/digital-competence-framework (accessed on 16 December 2020).

Eveleth, R. (2014), How Self-Tracking Apps Exclude Women, https://www.theatlantic.com/technology/archive/2014/12/how-self-tracking-apps-excludewomen/383673/.

Felps, W. et al. (2009), Turnover contagion: How coworkers' job embeddedness and job search behaviors influence quitting, Academy of Management Journal.

Gallup (2016), How Millennials Want to Live, https://enviableworkplace.com/wpcontent/uploads/Gallup-How-Millennials-Want-To-Work.pdf.

Gaucher, D.; Friesen, J; Kay, A. C. (2011), Evidence that gendered wording in job advertisements exists and sustains gender inequality, https://doi.org/10.1037/a0022530.

Gerson, D. (2020), "Leadership for a High Performing Civil Service: Towards senior civil service systems in OECD countries", OECD Working Papers on Public Governance, No. 40, OECD Publishing, Paris, https://dx.doi.org/10.1787/ed8235c8-en.

Government Digital Service (2020), Service designer, https://www.gov.uk/guidance/servicedesigner.

Government Digital Service (2019), Making the Digital, Data and Technology Capability Framework more user friendly, https://digitalpeople.blog.gov.uk/2019/10/24/making-the-digitaldata-and-technology-capability-framework-more-user-friendly/.

Government Digital Service, UK Cabinet Office (2019), Technology Code of Practice, https://www.gov.uk/government/publications/technology-code-of-practice/technology-code-ofpractice\#spend-controls-and-the-technology-code-of-practice.

Government of Canada (2020), Digital Academy, https://www.cspsefpc.gc.ca/About us/Business lines/digitalacademy-eng.aspx.

Gratton, L. (2016), "Rethinking the Manager's Role", MIT Sloan Management Review, https://sloanreview.mit.edu/article/technology-and-the-end-of-management/ (accessed on 
3 August 2020).

Guiso, L. and M. Paiella (2010), Risk aversion, wealth, and background risk, https://doi.org/10.1162/JEEA.2008.6.6.1109.

Hodge, B., W. Anthony and L. Gales (1991), Organization Theory: A Strategic Approach.

Kanten, P., S. Kanten and M. Gurlekc (2014), The Effects of Organizational Structures and Learning Organization on Job Embeddedness and Individual Adaptive Performance.

Klus, M. and J. Müller (2020), Identifying Leadership Skills Required in the Digital Age, http://www.RePEc.org (accessed on 1 August 2020).

Kratzer, J., R. Leenders and J. Van Engelen (2006), Managing creative team performance in virtual environments: an empirical study in $44 R \& D$ teams, https://www.academia.edu/23381694/Managing creative team performance in virtual enviro nments an empirical study in $44 \mathrm{R}$ and $\mathrm{D}$ teams.

Kubheka, I., P. Kholopane and C. Mbohwa (2013), The Effects of Flattening Hierarchies on Employee Performance in Organizations.

Kulkarni, S. and S. Scott (2019), Digital Acceleration: the Right C-Suite Expertise, https://www.heidrick.com/Knowledge-Center/Publication/Digital acceleration Theright $\mathrm{C}$ suite expertise.

Lean Enterprise Institute (2020), What's Lean?, https://www.lean.org/whatslean/.

Lewis, G. and S. Frank (2002), Who Wants to Work for the Government?, https://doi.org/10.1111/0033-3352.00193.

MacCormack, A., J. Rusnak and C. Baldwin (2008), Exploring the Duality between Product and Organizational Architectures: A Test of the Mirroring Hypothesis, https://hbswk.hbs.edu/item/5894.html.

Mergel, I., S. Ganapati and A. Whitford (2020), "Agile: A New Way of Governing”, Public Administration Review, http://dx.doi.org/10.1111/puar.13202.

Ministry of Public Administration (2020), Inovativen.si, https://www.gov.si/zbirke/projekti-inprogrami/inovativnost-v-javni-upravi-inovativen-si/.

Moss-Racusin, C. et al. (2012), Science faculty's subtle gender biases favor male students, https://doi.org/10.1073/pnas.1211286109.

Nova, A. (2020), Working from home? You might be able to expense a new desk, https://www.cnbc.com/2020/06/03/companies-are-paying-for-their-workers-home-offices.html.

OECD (2020), Digital Government in Chile - Improving Public Service Design and Delivery, OECD Digital Government Studies, OECD Publishing, Paris, https://dx.doi.org/10.1787/b94582e8-en.

OECD (2020), "Digital Government Index: 2019 results", OECD Public Governance Policy Papers, No. 3, OECD Publishing, Paris, https://dx.doi.org/10.1787/4de9f5bb-en.

OECD (2020), The COVID-19 Crisis: A catalyst for government transformation?, http://www.oecd.org/coronavirus/policy-responses/the-covid-19-crisis-a-catalyst-for- 
government-transformation-1d0c0788/.

OECD (2020), "The OECD Digital Government Policy Framework: Six dimensions of a Digital Government", OECD Public Governance Policy Papers, No. 02, OECD Publishing, Paris, https://dx.doi.org/10.1787/f64fed2a-en.

OECD (2019), Digital Government Review of Panama: Enhancing the Digital Transformation of the Public Sector, OECD Digital Government Studies, OECD Publishing, Paris, https://dx.doi.org/10.1787/615a4180-en.

OECD (2019), Going Digital: Shaping Policies, Improving Lives, OECD Publishing, Paris, https://dx.doi.org/10.1787/9789264312012-en.

OECD (2019), OECD Future of Education and Skills 2030: OECD Learning Compass 2030 - A Series of Concept Notes, OECD, Paris, France, http://www.oecd.org/education/2030project/teaching-and-learning/learning/learning-compass2030/OECD Learning Compass 2030 concept note.pdf.

OECD (2019), OECD Future of Education and Skills 2030: Student Angecy for 2030 - A Series of Concept Notes, https://www.oecd.org/education/2030-project/teaching-andlearning/learning/student-agency/Student Agency for 2030 concept note.pdf.

OECD (2019), OECD Skills Outlook 2019 : Thriving in a Digital World, OECD Publishing, Paris, https://dx.doi.org/10.1787/df80bc12-en.

OECD (2019), Recommendation of the Council on Public Service Leadership and Capability, OECD/LEGAL/0445, https://legalinstruments.oecd.org/en/instruments/OECD-LEGAL-0445.

OECD (2019), The Path to Becoming a Data-Driven Public Sector, OECD Digital Government Studies, OECD Publishing, Paris, https://dx.doi.org/10.1787/059814a7-en.

OECD (2018), Digital Government Review of Brazil: Towards the Digital Transformation of the Public Sector, OECD Digital Government Studies, OECD Publishing, Paris, https://dx.doi.org/10.1787/9789264307636-en.

OECD (2018), Promoting the Digital Transformation of African Portuguese-Speaking Countries and Timor-Leste, OECD Digital Government Studies, OECD Publishing, Paris, https://dx.doi.org/10.1787/9789264307131-en.

OECD (2017), Core Skills for Public Sector Innovation, https://www.oecd.org/media/oecdorg/satellitesites/opsi/contents/files/OECD OPSIcore skills for public sector innovation-201704.pdf.

OECD (2017), OECD Digital Economy Outlook 2017, OECD Publishing, Paris, https://dx.doi.org/10.1787/9789264276284-en.

OECD (2017), OECD Skills Outlook 2017: Skills and Global Value Chains, OECD Publishing, Paris, https://dx.doi.org/10.1787/9789264273351-en.

OECD (2017), Recommendation of the Council on Open Government, OECD/LEGAL/0438, OECD, Paris, https://legalinstruments.oecd.org/en/instruments/OECD-LEGAL-0438.

OECD (2017), Skills for a High Performing Civil Service, OECD Public Governance Reviews, OECD Publishing, Paris, https://dx.doi.org/10.1787/9789264280724-en. 
OECD (2017), The Pursuit of Gender Equality: An Uphill Battle, OECD Publishing, Paris, https://dx.doi.org/10.1787/9789264281318-en.

OECD (2015), Achieving Public Sector Agility at Times of Fiscal Consolidation, OECD Public Governance Reviews, OECD Publishing, Paris, https://dx.doi.org/10.1787/9789264206267-en.

OECD (2014), "Learning Begets Learning: Adult Participation in Lifelong Education", Education Indicators in Focus, No. 26, OECD Publishing, Paris, https://dx.doi.org/10.1787/5jxsvvmr9z8nen.

OECD (2014), Recommendation of the Council on Digital Government Strategies, OECD/LEGAL/0406, OECD, Paris, https://legalinstruments.oecd.org/en/instruments/OECDLEGAL-0406.

OECD (Forthcoming), Tackling the COVID-19 outbreak: Reinforcing the digital transformation of the public sector.

OECD, IDB (2016), Broadband Policies for Latin America and the Caribbean: a Digital Economy Toolkit, OECD Publishing, https://www.oecd-ilibrary.org/docserver/9789264251823-12en.pdf?expires $=1600951344 \&$ id $=i d \& a c c n a m e=0 c i d 84004878 \&$ checksum $=6 \mathrm{D} 24 \mathrm{~B} 2695 \mathrm{~F} 0943 \mathrm{~B}$ 5A573E80997280B74.

Partnership for 21st Century Skills (2008), 21st Century Skills, Education and Competitiveness: $A$ resource and policy guide, http://www.21stcenturyskills.org. (accessed on 3 August 2020).

Patall, E., H. Cooper and J. Robinson (2008), The Effects of Choice on Intrinsic Motivation and Related Outcomes: AMeta-Analysis of Research Findings, Duke University, http://dx.doi.org/10.1037/0033-2909.134.2.270.

Phillips, K., K. Liljenquist and M. Neale (2008), Is the Pain Worth the Gain? The Advantages and Liabilities of Agreeing With Socially Distinct Newcomers, https://doi.org/10.1177/0146167208328062.

Prestera, G. (2015), Push vs. Pull Learning, https://www.linkedin.com/pulse/push-vs-pull-learninggus-prestera/.

Ramsey, M. (2020), Hiring Challenges Confront Public-Sector Employers, https://www.shrm.org/hr-today/news/all-things-work/pages/hiring-challenges-confront-publicsector-employers.aspx.

Reljic, J., R. Evangelista and M. Pianta (2019), "Digital technologies, employment and skills", Laboratory of Economics and Management Working Paper Series, Scuola Superiore Sant'Anna, Pisa, http://www.growinpro.eu/.

Reuilly, P. (2004), Job Families: an integrating approach to reward and development, Institute for Employment Studies, https://www.employmentstudies.co.uk/system/files/resources/files/mp32.pdf.

Scrum.org (2020), What is Scrum?, https://www.scrum.org/resources/what-is-scrum.

SHRM (2020), Managing for Employee Retention, https://www.shrm.org/resourcesandtools/toolsand-samples/toolkits/pages/managingforemployeeretention.aspx.

Silva, A. (2016), What is Leadership ?, Journal of Business Studies Quarterly, https://media.proquest.com/media/pq/classic/doc/4225945211/fmt/pi/rep/NONE? s=tBsE\%2F 
msLDhGb\%2BtvMvtE1mTjaeSw\%3D.

Stansfield, N. (2015), Digital Show and Tell, https://digitalhealth.blog.gov.uk/2015/01/20/digitalshow-and-tell/.

Sullivan, G. (2014), Google statistics show Silicon Valley has a diversity problem, https://www.washingtonpost.com/news/morning-mix/wp/2014/05/29/most-google-employeesare-white-men-where-are-allthewomen/.

Teaching Public Service in the Digital Age (2020), The Competencies, https://www.teachingpublicservice.digital/competencies (accessed on 3 August 2020).

The Agile Organisation (2020), What is Agile Working?, https://www.agile.org.uk/what-is-agileworking/.

The Volcker Alliance (2020), Government-to-University Initiative, https://www.volckeralliance.org/G2U.

Thomas, D. (2017), Let's fund teams, not projects, https://defradigital.blog.gov.uk/2017/09/19/letsfund-teams-not-projects/.

Turqieh, G., H. Aoun and E. Nasr (2018), Agile in the public sector, https://www2.deloitte.com/xe/en/pages/about-deloitte/articles/we-are-25/agility.html.

UK Government Communication Service (2020), The impact of leadership behaviours on employee engagement, https://ic-space.gcs.civilservice.gov.uk/get-your-leadersengaging/evidence-to-make-the-case/.

van Laar, E. et al. (2017), "The relation between 21st-century skills and digital skills: A systematic literature review", Computers in Human Behavior, Vol. 72, pp. 577-588, http://dx.doi.org/10.1016/j.chb.2017.03.010.

Vuorikari, R. et al. (2016), Update Phase 1: The Conceptual Reference Model DigComp 2.0: The Digital Competence Framework for Citizens, European Commission, http://dx.doi.org/10.2791/11517.

Wenger-Trayner, E. and B. Wenger-Trayner (2015), Communities of practice a brief introdution, https://wenger-trayner.com/wp-content/uploads/2015/04/07-Brief-introduction-to-communitiesof-practice.pdf.

Winston, B. and K. Patterson (2006), An Integrative Definition of Leadership, https://www.regent.edu/acad/global/publications/ijls/new/vol1iss2/winston patterson.doc/winst on patterson.pdf.

World Economic Forum (2019), Al-assisted recruitment is biased. Here's how to make it more fair, https://www.weforum.org/agenda/2019/05/ai-assisted-recruitment-is-biased-heres-how-to-beatit/. 